\title{
Doubled $\alpha^{\prime}$-geometry
}

\section{Olaf Hohm, ${ }^{a}$ Warren Siegel ${ }^{b}$ and Barton Zwiebach ${ }^{c}$}

${ }^{a}$ Arnold Sommerfeld Center for Theoretical Physics, Theresienstrasse 37, D-80333 Munich, Germany

${ }^{b}$ C.N. Yang Institute for Theoretical Physics,

State University of New York, Stony Brook, NY 11794-3840, U.S.A.

${ }^{c}$ Center for Theoretical Physics, Massachusetts Institute of Technology,

Cambridge, MA 02139, U.S.A.

E-mail: olaf.hohm@physik.uni-muenchen.de, siegel@insti.physics.sunysb.edu, zwiebach@mit.edu

ABSTRACT: We develop doubled-coordinate field theory to determine the $\alpha^{\prime}$ corrections to the massless sector of oriented bosonic closed string theory. Our key tool is a string current algebra of free left-handed bosons that makes $\mathrm{O}(\mathrm{D}, \mathrm{D}) \mathrm{T}$-duality manifest. While T-dualities are unchanged, diffeomorphisms and $b$-field gauge transformations receive corrections, with a gauge algebra given by an $\alpha^{\prime}$-deformation of the duality-covariantized Courant bracket. The action is cubic in a double metric field, an unconstrained extension of the generalized metric that encodes the gravitational fields. Our approach provides a consistent truncation of string theory to massless fields with corrections that close at finite order in $\alpha^{\prime}$.

Keywords: Gauge Symmetry, Bosonic Strings, Conformal Field Models in String Theory, String Duality

ARXiv EPRINT: 1306.2970 


\section{Contents}

1 Introduction 2

1.1 History 2

1.2 Outline and summary 4

2 Doubled conformal field theory $\quad 9$

2.1 Double dimensions 9

2.2 Halving 11

3 Differential double geometry 12

3.1 Operators and contour integrals 12

$\begin{array}{lll}3.2 & \text { Bilinear operator products } & 13\end{array}$

$\begin{array}{lll}3.3 & \text { Cubic relations } & 16\end{array}$

4 Vector gauge symmetry $\quad 18$

$\begin{array}{lll}4.1 & \text { Inner and outer products, brackets } & 19\end{array}$

$\begin{array}{ll}4.2 \text { Evaluation } & 21\end{array}$

4.3 Jacobiator and N-tensor 23

4.4 Gauge transformations 25

5 Dilaton and double volume $\quad 26$

5.1 Virasoro operator $\mathcal{S} \quad 26$

5.2 Projection to divergence free tensors 29

6 Double metric 31

6.1 Field equations 31

$6.2 \star$ product 33

6.3 Action 36

6.4 Field equation evaluation 40

7 Relation to generalized metric formulation $\quad 41$

$\begin{array}{lll}7.1 & \text { Classical action } & 41\end{array}$

7.2 Double metric action and field equation 42

$\begin{array}{ll}7.3 & \text { Dilaton equation }\end{array}$

8 Prospects 46

$\begin{array}{ll}\text { A Quadratic identities } & 49\end{array}$

B Cubic identities $\quad 50$

C Evaluated products

D Alternate projection $\quad 52$ 


\section{Introduction}

\subsection{History}

The massless sector of the oriented, bosonic, closed string consists of the graviton (metric), "axion" or "notoph" (2-form gauge field), and dilaton. The T-duality symmetry of the Ddimensional theory with $\mathrm{d}$ compactified dimensions is $\mathrm{O}(\mathrm{d}, \mathrm{d})$, over the integers for the full string theory but over the reals for the massless sector. The string theory, of course, lives in $\mathrm{D}=26$, but the massless theory we are studying exists for all values of $\mathrm{D}$. Duality transforms the $(\mathrm{D}-\mathrm{d})$-dimensional scalars resulting from dimensional reduction of the metric and 2form, but leaves invariant the dilaton, when defined as a scalar density. This is the usual treatment of T-duality when winding modes are ignored and dimensional reduction is described in the language of Killing vectors that imply the independence of the background from the d compact coordinates.

This $\mathrm{O}(\mathrm{d}, \mathrm{d})$ can be represented on these scalars in terms of a nonlinear $\sigma$-model for the coset $\mathrm{O}(\mathrm{d}, \mathrm{d}) / \mathrm{O}(\mathrm{d}) \times \mathrm{O}(\mathrm{d})[7]$. But this approach can be generalized [10-12] in a way that:

a) requires no dimensional reduction,

b) includes the full set of massless fields,

c) includes all gauge invariances,

d) defines covariant derivatives (connections, torsions, curvatures, Bianchi identities), and

e) manifests a full $\mathrm{O}(\mathrm{D}, \mathrm{D})$ symmetry on the fields, gauge invariances, and action.

This procedure doubles the coordinates on which all fields depend. The reduction to $D$ dimensions is achieved by a set of constraints that preserves the manifest $O(D, D)$, but any solution of the constraints "spontaneously breaks" this symmetry down to the usual $\mathrm{O}(\mathrm{D}-1,1)$ Lorentz symmetry, reproducing the standard D-dimensional fields, gauge invariances, and action. The $\mathrm{O}(\mathrm{d}, \mathrm{d})$ can then be restored manifestly by compactification, which weakens the constraints.

The left- and right-handed worldsheet currents (affine Lie algebra) form the defining representation of this $\mathrm{O}(\mathrm{D}, \mathrm{D})$. Through coupling quadratically to these currents, the metric and 2-form combine to form the coset $\mathrm{O}(\mathrm{D}, \mathrm{D}) / \mathrm{O}(\mathrm{D}-1,1)^{2}$. The action can be expressed in a manifestly $\mathrm{O}(\mathrm{D}, \mathrm{D})$ covariant form in terms of this field and the dilaton, which acts as the spacetime integration measure.

In more recent developments [14] the construction of such a double field theory was based on closed string field theory $[15,16]$. This work identified the constraints mentioned above as the strong version of the $L_{0}-\bar{L}_{0}=0$ level-matching condition of closed string fields. In its standard and seemingly unavoidable (weak) form, it applies to all fields and gauge parameters. In the strong version, which demands that all products of fields are also killed by $L_{0}-\bar{L}_{0}$, it provides the reduction to D dimensions. While the construction could be carried to cubic order in fluctuations without imposing the strong constraint, the full 
construction became tractable only once this constraint is imposed. In this case the string field gauge algebra is governed by the bracket anticipated in [11]: the C-bracket, which was shown [17] to be the duality-covariantized version of the Courant bracket of generalized geometry. This suggested the possibility of a compact explicit form of the doubled action in terms of the generalized metric $\mathcal{H}_{M N}$ and the duality invariant dilaton, a construction provided in [18]. The simplicity of the action allows quick confirmation that any solution of the strong constraint gives precisely the two-derivative action for the massless sector of closed string theory. An alternative form of the action in terms of the field $\mathcal{E}_{i j}=(g+b)_{i j}$ was given earlier in [19].

There is also a compelling generalization of Riemannian geometry for this dualitycovariant framework. A complete formulation has been given in [11] in a frame-like formalism, including torsions, curvatures (Riemann tensor), differential Bianchi identities, and a discussion of the ambiguity of some Lorentz connections and curvatures. In [20] this formalism has been related to the double field theory actions of $[18,19]$ and to a metriclike formulation. The metric-like approach has been examined in more detail in $[24,25]$ (in a "semi-covariant" approach that truncates connections) and in [21]. The fully "invariant" formulation in [23] provides a unifying framework for the metric- and frame-like formalisms. This includes an index-free definition of the torsion and Riemann tensor, a complete algebraic Bianchi identity with torsion, and a discussion of the absence of an uncontracted differential Bianchi identity. This geometry is related to (and an extension of) the "generalized geometry" of Hitchin and Gualtieri [26-28].

Formulations including the coupling to vector multiplets, relevant for heterotic and type I strings, were also given in $[11,12]$ and worked out in the generalized metric formulation in [30]. The $\mathcal{N}=1$ supersymmetric form is contained in the superspace results of $[11,12]$ and was worked out independently in explicit component form in [34]. (See also [35] for supersymmetric double field theory without vector multiplets.) The RamondRamond sector of type-II superstrings is given in [31, 32], and its supersymmetric extension in [33].

Double field theory formulations where the strong constraint is somewhat relaxed have been given for massive IIA supergravity in [29], for flux compactifications in [37, 38], and explored in some generality in [39, 40]. See also [41, 42] for the geometric role of nongeometric fluxes in double field theory. Global aspects of double field theory are discussed in [22] where a formula for large gauge transformations was proposed and examined in detail. There are numerous other developments in double field theory and the closely related M-theory (see [43-45]); for a recent review with further references see [46].

One of the most intriguing features of the theory is the absence of a satisfactory duality-covariant generalized Riemann tensor. In the geometric formalism the covariant constraints do not suffice to determine all components of the connections in terms of physical fields, resulting in a Riemann tensor with some undetermined components. In fact, the undetermined components of the generalized Riemann tensor are such that this tensor encodes nothing more than the Ricci curvature and scalar curvature [11, 21, 23].

It has been known for some time that $\alpha^{\prime}$ corrections to the massless effective field theory preserve the T-duality symmetry of the two-derivative action [47]. This has been 
verified explicitly in [48] to first order in $\alpha^{\prime}$ in a reduction down to just one dimension. The $\alpha^{\prime}$ corrections to the action of bosonic closed strings include Riemann-squared terms. In the absence of a duality-covariant Riemann tensor, it has been hard to imagine how one could describe $\alpha^{\prime}$ corrections in a manifestly duality invariant way. For example, it was seen in [21] that certain structures in Riemann-squared cannot be written in terms of the generalized metric. This lack of a suitable duality-covariant Riemann tensor is a clear indication that some symmetries of the theory must receive $\alpha^{\prime}$ corrections. While the one-dimensional results of [48] suggest that gauge symmetries could be corrected, the more accepted viewpoint has been that $\alpha^{\prime}$ corrections to the T-duality transformations are required. These, however, have been hard to determine, even for the case of compactifications over a single circle and to first order in $\alpha^{\prime}$ [49].

On the other hand, the string field theory based analysis [14] is by construction duality covariant (although background dependent), suggesting again that duality need not be corrected. It was noted in [23], moreover, that the gauge symmetry brackets calculated to lowest order in derivatives in [14] receive computable $\alpha^{\prime}$ corrections. These corrections have been determined, appear to agree with the results to be presented here, and will be considered elsewhere as supporting evidence for the connection to string theory. It was simpler, however, to approach the construction by extending the current algebra methods developed in [10-12] and this is what we will do in this paper.

\subsection{Outline and summary}

In this paper the main technical tool is a modified worldsheet theory that amounts to a certain consistent truncation of string theory. We will have $\mathrm{D}+\mathrm{D}$ bosonic worldsheet fields $X^{M}(M=1,2, \ldots, 2 D)$ of one handedness, instead of the familiar fields $X^{i}(z)$ and $X^{i}(\bar{z})$, with $i=1, \ldots, D$. In this formulation there is a chirality condition setting momenta equal to $z$-derivatives of coordinates: $P^{M}=X^{\prime M} \equiv Z^{M}$. There is also a constraint the strong constraint - that must be satisfied by the functions of $X^{M}$ that are used to describe background fields. These fields and their products must be annihilated by the differential operator $\eta^{M N} \partial_{M} \partial_{N}$, where $\partial_{M}=\partial / \partial X^{M}$ and $\eta$ is the $\mathrm{O}(\mathrm{D}, \mathrm{D})$ metric. This simplified version of the string truncates the $\alpha^{\prime}$ corrections of the full string theory, which is evident from the fact that all operator products terminate. We will see this as we obtain the equations of motion for the background fields. Analysis indicates that this truncation duplicates string theory to cubic order in fields.

In this paper we use the quantum mechanical approach to string theory, not the quantum field theory approach. Hence "quantum" in this context will always refer to the JWKB approximation in orders of $\alpha^{\prime}$. Our main goal, of course, is the construction of a classical double field theory, a space-time field theory which includes $\alpha^{\prime}$ corrections to the twoderivative theory. Perhaps this double field theory is the string field theory that results from the modified worldsheet theory.

We extend the current algebra methods of [10-12] to a full-fledged discussion of the worldsheet conformal field theory, including propagators

$$
\left\langle X^{M}\left(z_{1}\right) X^{N}\left(z_{2}\right)\right\rangle=\eta^{M N} \ln \left(z_{1}-z_{2}\right),
$$


and the associated operator product expansions in section 2. Note the appearance in the above right-hand side of the $\mathrm{O}(\mathrm{D}, \mathrm{D})$ metric, at the place where the familiar theory uses the space-time metric. A major simplification is that the strong constraint implies that there are no singular terms in the operator product $A(X) B(X)$ of any two $X$-dependent fields. In this world-sheet theory we consider explicitly three kinds of operators: scalars $f$, vectors $V$, and tensors $T$

$$
\begin{aligned}
f & =f(X) \\
V & =V^{M}(X) Z_{M} \\
T & =\frac{1}{2} T^{M N}(X) Z_{M} Z_{N}-\frac{1}{2}\left(\hat{T}^{M} Z_{M}\right)^{\prime} .
\end{aligned}
$$

The above are operators of conformal weight zero, one, and two, respectively. The tensor requires the two terms shown for the closure of the algebra of operator products. We refer to them as the two-index component and the one-index component (or pseudovector part) of the tensor.

We find it useful to treat operator products systematically in section 3. Given two operators $\mathcal{O}_{1}$ and $\mathcal{O}_{2}$, the product $\mathcal{O}_{1} \circ_{w} \mathcal{O}_{2}$, with $w \geq 0$ an integer, is an operator of weight $w$ that appears in the operator product of $\mathcal{O}_{1}\left(z_{1}\right) \mathcal{O}_{2}\left(z_{2}\right)$ as follows

$$
\mathcal{O}_{1}(1) \mathcal{O}_{2}(2)=\sum_{w=0}^{\infty} \frac{1}{\left(z_{12}\right)^{w_{1}+w_{2}-w}}\left(\mathcal{O}_{1} \circ_{w} \mathcal{O}_{2}\right)(2)
$$

Here and in the following we use the short-hand notation $z_{1} \equiv 1$, etc. The product $\mathcal{O}_{1} \circ_{0} \mathcal{O}_{2}$ is a scalar and will be written as the inner product $\left\langle\mathcal{O}_{1} \mid \mathcal{O}_{2}\right\rangle$. We examine various infinite classes of identities satisfied by these products. In general the products do not have definite symmetry properties, but there are symmetry relations.

Vector operators $\Xi=\xi^{M}(X) Z_{M}$ generate gauge transformations (section 4). The components $\xi^{M}(X)$ of the operator comprise $\mathrm{D}+\mathrm{D}$ gauge parameters $\xi^{i}$ and $\tilde{\xi}_{i}$. The operator product expansion $\Xi_{1}\left(z_{1}\right) \Xi_{2}\left(z_{2}\right)$ of two such vector operators, with parameters $\xi_{1}^{M}$ and $\xi_{2}^{M}$, defines fundamental structures of the theory. We get the inner product $\left\langle\Xi_{1} \mid \Xi_{2}\right\rangle$ as the residue of the second-order pole. This is a symmetric, bilinear scalar operator that takes the form

$$
\left\langle\Xi_{1} \mid \Xi_{2}\right\rangle=\xi_{1}^{M} \xi_{2}^{N} \eta_{M N}-\left(\partial_{N} \xi_{1}^{M}\right)\left(\partial_{M} \xi_{2}^{N}\right) .
$$

The first term is familiar from the classical theory and the second term is the $\alpha^{\prime}$ correction, arising from a quantum contribution in the OPE. Since we do not write explicitly $\alpha^{\prime}$ factors, corrections are recognized by the increased number of space-time derivatives. We get a vector operator $\left[\Xi_{1}, \Xi_{2}\right]_{C}$ as the residue of the first-order pole. Its components take the form

$$
\left[\Xi_{1}, \Xi_{2}\right]_{C}^{M}=\xi_{[1}^{N} \partial_{N} \xi_{2]}^{M}-\frac{1}{2} \xi_{1}^{K} \stackrel{\leftrightarrow}{\partial}{ }^{M} \xi_{2 K}+\frac{1}{2}\left(\partial_{K} \xi_{1}^{L}\right) \stackrel{\leftrightarrow}{\partial}^{M}\left(\partial_{L} \xi_{2}^{K}\right)
$$

(In this paper we use the (anti)symmetrization convention $[a b]=a b-b a$, and $A \overleftrightarrow{\partial} B=$ $A \partial B-(\partial A) B$.) The first term on the right-hand side is the Lie bracket of vector fields. 
Together with the next term it defines the "classical" C -bracket, the duality covariantized version of the Courant bracket of generalized geometry. The last term, with three derivatives, is the new nontrivial correction. The strong constraint implies that no higher derivative correction to the bracket can be written that is, as required, linear in each of the gauge parameters. This correction is therefore unique. Moreover, the bracket is fully consistent: its Jacobiator is a trivial gauge parameter, just as it was for the classical C bracket. A trivial gauge parameter does not generate gauge transformations and takes the form of the $z$-derivative $f^{\prime}$ of a scalar operator $f$. The quantum C-bracket given above defines the algebra of gauge transformations in the theory we construct here.

Associated to Courant structures of generalized geometry there are Dorfman structures that are often more convenient. For us, C-type operators have D-type counterparts. Amusingly, $\mathrm{C}$ operators arise by presenting the operator product expansion symmetrically in $z_{1}$ and $z_{2}$, while their $\mathrm{D}$ counterparts arise by presenting the expansion with operators based at $z_{2}$. The vector operator $\left[\Xi_{1}, \Xi_{2}\right]_{D}$ is the quantum $\mathrm{D}$ bracket, whose classical version is the duality covariantized Dorfman bracket.

Very nontrivially, the above corrections do not vanish upon reduction from $\mathrm{D}+\mathrm{D}$ to $\mathrm{D}$ dimensions, as done by setting $\tilde{\partial}^{i}$ derivatives to zero. For the inner product we get

$$
\left\langle\Xi_{1} \mid \Xi_{2}\right\rangle=\xi_{1}^{i} \tilde{\xi}_{2 i}+\xi_{2}^{i} \tilde{\xi}_{1 i}-\partial_{i} \xi_{1}^{j} \partial_{j} \xi_{2}^{i}
$$

The last term is the quantum correction. For the $\mathrm{C}$ bracket the vector part is not corrected, but the one-form part is:

$$
\left(\left[\Xi_{1}, \Xi_{2}\right]_{C}\right)_{i}=\ldots+\frac{1}{2}\left(\partial_{k} \xi_{1}^{\ell}\right) \stackrel{\leftrightarrow}{\partial}_{i}\left(\partial_{\ell} \xi_{2}^{k}\right),
$$

where the dots denote the contributions from the "classical terms". Therefore our results go beyond generalized geometry in that the familiar inner product and the Courant bracket are deformed.

Gauge transformations $\delta_{\xi} \mathcal{O}$ of any operator $\mathcal{O}$ are defined by the commutator $\delta_{\xi} \mathcal{O}=$ $\left[\int \Xi, \mathcal{O}\right]$, and are readily evaluated with the use of operator products. For a vector operator $V$, for example,

$$
\delta_{\xi} V^{M}=\xi^{P} \partial_{P} V^{M}+\left(\partial^{M} \xi_{P}-\partial_{P} \xi^{M}\right) V^{P}-\left(\partial^{M} \partial_{K} \xi^{L}\right) \partial_{L} V^{K} .
$$

The last term is the quantum correction. In $\mathrm{D}$ dimensions, the quantum correction vanishes for the transformation $\delta_{\xi} V^{i}$ of a vector but does not vanish for the transformation $\delta_{\xi} V_{i}$ of the one-form (see (4.44)). In mathematical language this represents a deformation of generalized Lie derivatives.

With the gauge structure defined, the fields of the theory are introduced using a pair of tensor operators. We start with $\frac{1}{2} Z^{2} \equiv \frac{1}{2} \eta^{M N} Z_{M} Z_{N}$, the analog of the Virasoro operator $T_{\sigma}$ that in the undoubled flat-space theory is proportional to $X^{\prime i} P_{i}$. We then introduce in section 5 the dilaton in a tensor $\mathcal{S}$ defined to be

$$
\mathcal{S} \equiv \frac{1}{2}\left(Z^{2}-\phi^{\prime \prime}\right)
$$


The second term is consistent with the general form in (1.2) since $\phi^{\prime \prime}=\left(Z^{M} \partial_{M} \phi\right)^{\prime}$ (recall $\left.Z=X^{\prime}\right)$. This dilaton improvement is needed for consistency of gauge transformations. As it turns out, the gauge transformation of the dilaton receives no quantum corrections (see $(5.5))$.

The products also satisfy useful distributive type identities. Products of the dilatonbased tensor $\mathcal{S}$ with a tensor $T$ lead to convenient definitions

$$
\mathcal{S} \circ_{0} T=\frac{1}{2} \operatorname{tr} T, \quad \mathcal{S} \circ_{1} T=\operatorname{div} T .
$$

The trace of a tensor is a scalar with leading term $\eta^{M N} T_{M N}$. The divergence of a tensor is a vector with leading term $\partial_{N} T^{M N}$. Both have nontrivial $\alpha^{\prime}$ contributions that can be seen in (5.17).

A second tensor operator $\mathcal{T}$ is used in section 6 to introduce the gravitational fields, metric and two-form. This operator is the analog of the Virasoro operator $T_{\tau}$ that in the undoubled flat-space theory is proportional to $\left(P_{i}\right)^{2}+\left(X^{\prime i}\right)^{2}$. In toroidal backgrounds, this operator is a quadratic form on currents with the generalized metric used to contract indices. In our formulation we start with a double metric $\mathcal{M}^{M N}$ that will turn out to be related but not equal to the generalized metric $\mathcal{H}^{M N}$. While off-shell the latter squares to one, the former is unconstrained. The tensor operator $\mathcal{T}$ takes the form

$$
\mathcal{T} \equiv \frac{1}{2} \mathcal{M}^{M N} Z_{M} Z_{N}-\frac{1}{2}\left(\widehat{\mathcal{M}}^{M} Z_{M}\right)^{\prime} .
$$

The second term, needed for consistency with gauge transformations, contains a field $\widehat{\mathcal{M}}^{M}$, to be determined in terms of the double metric and the dilaton. The gauge transformation of the double metric $\mathcal{M}^{M N}$ receives $\alpha^{\prime}$ and $\alpha^{\prime 2}$ corrections (see (6.39)).

Having introduced the dilaton and the double metric on the weight-two tensor operators $\mathcal{S}$ and $\mathcal{T}$, we make the usual assumption that the equations of motion of these fields are the conditions that $\mathcal{S}$ and $\mathcal{T}$ form the Virasoro algebra:

$$
\begin{aligned}
& \mathcal{S}(1) \mathcal{S}(2)=\frac{D}{z_{12}^{4}}+\frac{2 \mathcal{S}(2)}{z_{12}^{2}}+\frac{\mathcal{S}^{\prime}(2)}{z_{12}}+\text { finite } \\
& \mathcal{S}(1) \mathcal{T}(2)=\frac{2 \mathcal{T}(2)}{z_{12}^{2}}+\frac{\mathcal{T}^{\prime}(2)}{z_{12}}+\text { finite } \\
& \mathcal{T}(1) \mathcal{T}(2)=\frac{D}{z_{12}^{4}}+\frac{2 \mathcal{S}(2)}{z_{12}^{2}}+\frac{\mathcal{S}^{\prime}(2)}{z_{12}}+\text { finite }
\end{aligned}
$$

Remarkably, the operator product $\mathcal{S S}$ (first line) works out automatically without imposing any condition on the dilaton. This is required, since the dilaton equation of motion involves the double metric, which does not appear in $\mathcal{S}$. For the $\mathcal{S} \mathcal{T}$ operator product (second line) the terms on the right-hand side appear as expected, but the vanishing of the quartic and cubic poles give nontrivial conditions. In the notation of (1.10) these correspond to

$$
\operatorname{tr} \mathcal{T}=0 \text { and } \operatorname{div} \mathcal{T}=0 .
$$

The first equation is the $\alpha^{\prime}$-corrected equation of motion of the dilaton. The second equation determines the auxiliary field $\widehat{\mathcal{M}}^{M}$ in terms of double metric and the dilaton. For the 
$\mathcal{T} \mathcal{T}$ operator product (third line), we prove that the only nontrivial conditions are getting a constant quartic pole and the correct value for the quadratic pole. In terms of products,

$$
\langle\mathcal{T} \mid \mathcal{T}\rangle=\text { constant }, \quad \mathcal{T} \circ_{2} \mathcal{T}=2 \mathcal{S}
$$

The second condition is a tensor equation and its two-index part is the double metric equation of motion. In terms of the matrix $\mathcal{M}^{M N}$ it takes the form $\mathcal{M}^{2}=1+2 \mathcal{V}$, where $\mathcal{V}$ is quadratic in $\mathcal{M}$ and contains from two up to six derivatives. While the generalized metric squares to the identity, the double metric squares to the identity plus higher derivatives terms. We view this as a most significant departure from the classical theory, forced by $\alpha^{\prime}$ corrections. We prove that the first equation in (1.14) as well as the one-index part of the second equation are redundant.

The construction of the action is done in terms of the tensor operators $\mathcal{S}$ and $\mathcal{T}$, with the latter constrained to have zero divergence. These operators encode the double metric $\mathcal{M}^{M N}$ and the dilaton. We examine the properties of divergence-free tensors and introduce an "overline" projector that acting on a weight-two tensor $T$ gives a tensor $\bar{T}$ with $\operatorname{div} \bar{T}=0$. Using this projection we define a $\star$-product mapping into 2 -tensors such that $T_{1} \star T_{2}=T_{2} \star T_{1}$ is divergenceless. We are then able to write a manifestly gauge invariant and $\mathrm{O}(\mathrm{D}, \mathrm{D})$ invariant action

$$
S=\int e^{\phi}\left[\langle\mathcal{T} \mid \mathcal{S}\rangle-\frac{1}{6}\langle\mathcal{T} \mid \mathcal{T} \star \mathcal{T}\rangle\right]
$$

This action is cubic in the double metric (with no quadratic term!) and contains up to six derivatives. We show by variation that the expected equations of motion arise. This uses a key property of the star product: the complete symmetry of $\int e^{\phi}\left\langle\bar{T}_{1} \mid \bar{T}_{2} \star \bar{T}_{3}\right\rangle$ under the exchange of any pair of $\bar{T}$ 's. The dilaton equation of motion also emerges correctly, but takes a bit more effort since dilaton variations affect the overline projection and thus $\delta_{\phi} \mathcal{T}$ is not divergence free.

We work out explicitly the above action in section 7 , including all terms with up to two derivatives and confirm that the generalized metric form of the two-derivative action emerges. This reassuring confirmation provides an explicit test for many of our formulae. The above action almost certainly encodes Riemann-squared and Riemann-cubed corrections to the two-derivative action, but we will leave a direct verification of this for future work. For sure, we have constructed a completely consistent and exactly gauge invariant $\alpha^{\prime}$-deformation of the low-energy effective action. The action contains bounded powers of $\alpha^{\prime}$, at least when written in terms of the gravitational variable $\mathcal{M}$ and the dilaton. It thus seems unlikely that this is the full string effective field theory of the massless sector. We believe, instead, that this theory is a consistent truncation of string theory in which some of the stringy non-locality has been eliminated.

Our paper concludes with some perspectives on the results and discussion of open questions. 


\section{Doubled conformal field theory}

\subsection{Double dimensions}

We first describe the construction in double dimensions, then show how it reduces to the usual D dimensions. We introduce the gauge-invariant constant metric $\eta_{M N}$ of $\mathrm{O}(\mathrm{D}, \mathrm{D})$, which we use implicitly to raise/lower and contract $\mathrm{O}(\mathrm{D}, \mathrm{D})$ indices $M, N$. We also have $2 D$ chiral fields $X^{M}(z)$ representing the doubled coordinates. Then the $\mathrm{D}+\mathrm{D}$ dimensional formalism is described by the constraints

$$
\begin{aligned}
\text { Halving: } \quad \text { strong: } & \left(\partial^{M} A\right)\left(\partial_{M} B\right)=\partial^{M} \partial_{M} A=0, \\
\text { chirality: } & P^{M}=X^{M} \equiv Z^{M} \Rightarrow A(X)^{\prime}=Z^{M} \partial_{M} A .
\end{aligned}
$$

The first line is the duality-invariant strong constraint on fields or gauge parameters $A, B$, which are by definition functions of $X^{M}$. The constraint states that they, as well as their products, must be annihilated by $\partial^{M} \partial_{M}$. The chirality condition halves the number of oscillators in the theory by setting $P_{M}$, the canonical conjugate to $X^{M}$, equal to $X_{M}^{\prime}$. This current is denoted as $Z_{M}$ and appears each time we take $z$-derivatives (denoted by prime) of $X$-dependent operators.

We will also have Virasoro operators $\mathcal{S}$ and $\mathcal{T}$ that must have zero expectation values on physical states:

$$
\begin{array}{ll}
\text { "Virasoro": } & \mathcal{S}: \frac{1}{2} Z^{2}+\mathcal{O}\left(\alpha^{\prime}\right)=0 \\
& \mathcal{T}: \frac{1}{2} \mathcal{M}_{M N}(X) Z^{M} Z^{N}+\mathcal{O}\left(\alpha^{\prime}\right)=0 .
\end{array}
$$

The explicit construction of these operators will be discussed later, and only leading terms have been shown above. The background field $\mathcal{M}$ is the double metric, an extension of the generalized metric, and will play an important role in our theory. It should be emphasized that neither the strong constraint nor the chirality condition acquire $\alpha^{\prime}$ corrections.

We use the Hamiltonian formalism: the above constraints can be imposed at fixed $\tau$ (but will be preserved at all $\tau$ ). The halving constraints will be used immediately for reduction to the usual D X's. The Virasoro constraints will have the usual interpretation in D dimensions, but not in $\mathrm{D}+\mathrm{D}$ : because of the chirality constraint, only half of the energymomentum tensor should survive, yet we still impose two sets of similar constraints.

(Note that by "chiral", as referring to the $X^{M}$, we mean left-handed only, i.e., no "antichiral". Chiral bosons were described in Lagrangian language in $[1,6]$. In nonunitary gauges, such actions can be reduced to the usual $\phi \square \phi[1]$ or to $\phi \partial_{\sigma}\left(\partial_{\sigma}-\partial_{\tau}\right) \phi[5]$, resulting in a second nonchiral set of modes that must be removed as usual by the first-class constraints implied by the original gauge invariance, which must be preserved by the interactions. Bosons of both chiralities, D left $+\mathrm{D}$ right, were used in $[8,9]$, but T-duality was considered only for constant backgrounds, i.e., $\mathrm{d}=\mathrm{D}$, and thus all fields were compactification scalars.)

As will be elaborated in section 4, gauge transformations of an operator $T$ (inducing the transformation of the fields contained in $T$ ) are to be computed by the commutator

$$
\delta_{\xi} T=\left[\int \Xi, T\right], \quad \Xi=\xi_{M}(X) Z^{M},
$$


where $\xi_{M}$ are the gauge parameters. (Here " $\int$ " means " $\int d z / 2 \pi i$ ". This is essentially an integral over all $\sigma$ for constant $\tau$. In radial quantization it's an integral enclosing the origin. We'll use " $\oint$ " for closed contours not enclosing the origin.)

In previous work the focus was on equal- "time" $(\tau)$ commutation relations and only Poisson brackets were used. Here we find it convenient to introduce operator products, and therefore time dependence. We therefore choose the Hamiltonian

$$
H=\int d \sigma \mathcal{S}=\int d \sigma \frac{1}{2} Z^{2}
$$

We'll see later that the quantum corrections to $\mathcal{S}$ are a total derivative, so $H$ has no corrections. This Hamiltonian is background independent, thus very different from the familiar background-dependent D-dimensional Hamiltonian. We also have the equal- $\tau$ commutation relations

$$
\left[Z^{M}\left(\tau, \sigma_{1}\right), Z^{N}\left(\tau, \sigma_{2}\right)\right]=-i \eta^{M N} \delta^{\prime}\left(\sigma_{2}-\sigma_{1}\right) .
$$

The Heisenberg equation of motion for the operators $Z_{M}$ then takes the form

$$
i \partial_{\tau} Z^{M}(\tau, \sigma)=\left[Z^{M}(\tau, \sigma), H\right]=i \partial_{\sigma} Z^{M}(\tau, \sigma)
$$

so that $Z^{M}$ is a chiral field:

$$
\left(\partial_{\sigma}-\partial_{\tau}\right) Z^{M}=0 .
$$

The $X^{M}$ are thus chiral fields as well. We therefore have the propagator (back in the complex plane)

$$
\left\langle X^{M}(1) X^{N}(2)\right\rangle=\eta^{M N} \ln z_{12},
$$

where $z_{12}=z_{1}-z_{2}$. An $\alpha^{\prime}$ is needed on the right-hand side for proper dimensions. For simplicity, however, we will set $\alpha^{\prime}=1$. Note that the sign of $\alpha^{\prime}$ is arbitrary: we can freely replace $\eta \rightarrow-\eta$, since it's the indefinite metric of $\mathrm{O}(\mathrm{D}, \mathrm{D})$ anyway. From the above propagator and the identification of $Z$ with $X^{\prime}$ follow the operator products

$$
\begin{gathered}
Z_{M}(1) Z_{N}(2)=\frac{1}{z_{12}^{2}} \eta_{M N}+\text { finite }, \\
Z_{M}(1) A(X(2))=\frac{1}{z_{12}} \partial_{M} A(2)+\text { finite } .
\end{gathered}
$$

A remarkable simplification occurs due to the strong constraint: There are no singular terms in the OPE of fields. Indeed, on general grounds

$$
A(1) B(2)=: A(1) e^{\overleftarrow{\partial}_{M}\left\langle X^{M}(1) X^{N}(2)\right\rangle \partial_{N}} B(2):
$$

as seen, e.g., by Fourier transformation of the fields

$$
A(X(1)) \equiv \int d k_{1} e^{i k_{1} \cdot X(1)} \tilde{A}\left(k_{1}\right), \quad B(X(2)) \equiv \int d k_{2} e^{i k_{2} \cdot X(2)} \tilde{B}\left(k_{2}\right),
$$

and using the identity

$$
e^{i k_{1} \cdot X(1)} e^{i k_{2} \cdot X(2)}=: e^{i k_{1} \cdot X(1)} e^{i k_{2} \cdot X(2)}: e^{-k_{1 M} k_{2 N}\left\langle X^{M}(1) X^{N}(2)\right\rangle} .
$$


Using the propagator (2.8) and then the strong constraint, (2.10) gives

$$
A(1) B(2)=: A(1) e^{\overleftarrow{\partial}^{M} \ln \left(z_{12}\right) \partial_{M}} B(2):=: A(1) B(2):
$$

The result is conceptually clear: the propagator couples coordinates to their duals and strongly constrained fields never depend on both a coordinate and its dual. Since $X$ 's without derivatives occur only as arguments of fields, it follows from (2.13) that no explicit $(\ln z)$ 's will appear in our contractions. This situation is similar to the treatment of the twistor superstring formalism for $\mathrm{N}=4$ super Yang-Mills as a closed string with chiral worldsheet fields [13]. There the absence of ln's corresponds to the fact that the theory describes only particles and not true strings.

\subsection{Halving}

For reduction to D dimensions, we use the strong constraint to reduce the dependence of fields to half the coordinates, thus essentially eliminating half the zero-modes. (For this paper we do not compactify, so these constraints eliminate winding modes.) We then use the chirality constraint to eliminate half the oscillator modes: writing the metric as

$$
\eta_{M N}=\left(\begin{array}{cc}
0 & \delta_{m}^{n} \\
\delta_{n}^{m} & 0
\end{array}\right),
$$

in terms of the usual D-valued spacetime indices $m$, we have

$$
\begin{aligned}
\text { chirality: } & Z^{M} \rightarrow\left(X^{\prime m}, P_{m}\right) \\
\text { strong: } & X^{M} \rightarrow\left(X^{m}, 0\right)
\end{aligned}
$$

where the latter refers to the arguments of fields, the only place $X$ doesn't appear as $Z$. Solving the halving constraints in terms of the usual D coordinates, the Virasoro constraints can then be recognized as the usual (in Hamiltonian formalism).

With the above conditions, we have $H=\int d \sigma X^{\prime m} P_{m}$ and the associated action $S_{H}$ in Hamiltonian form is given by

$$
S_{H}=\int d^{2} \sigma P_{m}\left(\partial_{\tau}-\partial_{\sigma}\right) X^{m}
$$

whose counterpart in Lagrangian language has the singular form

$$
S_{L} \sim \lim _{\epsilon \rightarrow 0} \frac{1}{\epsilon} \int d^{2} \sigma \frac{1}{2}\left[\left(\partial_{\tau}-\partial_{\sigma}\right) X\right]^{2} .
$$

Using the worldsheet metric, the usual string action in D dimensions (without dilaton) takes the Hamiltonian form

$$
S_{H} \sim \int d^{2} \sigma\left(\dot{X}^{m} P_{m}-\frac{\sqrt{-g}}{g_{11}} \frac{1}{2} \mathcal{M}_{M N} Z^{M} Z^{N}-\frac{g_{01}}{g_{11}} \frac{1}{2} Z^{2}\right)
$$

Then the action (2.16) corresponds to the singular gauge

$$
\frac{\sqrt{-g}}{g_{11}}=0 \quad, \quad \frac{g_{01}}{g_{11}}=1
$$

a fact that may eventually be used to explain that the theory is some kind of $\alpha^{\prime}$ truncation of the full string theory (as stated at the end of the introduction). 


\section{$3 \quad$ Differential double geometry}

\subsection{Operators and contour integrals}

Although we will focus in the following sections on operators of lower conformal weight, we provide here a general pedagogical discussion, relating the different applications and their future use. We thus consider general operators consisting of functions of $X$ (evaluated at some value of $z$ ), carrying arbitrary numbers of $(\mathrm{D}+\mathrm{D})$-valued indices, all contracted with $Z$ 's and their $z$-derivatives. We define the conformal weight " $w$ " of such an operator (eigenvalue of " $\tilde{w}$ ") as the number of $Z$ 's plus the number of primes $(')$ :

$$
\tilde{w}(Z)=\tilde{w}\left({ }^{\prime}\right)=1, \quad \tilde{w}(X)=0 .
$$

Any of the operators to be considered has definite weight, but may consist of general linear combinations of terms of that weight, which differ by how that weight comes from $Z$ 's vs. primes. (This definition of weight agrees with the conformal field theory definition of weight when operators are on-shell.) The lowest weight operators, which play a central role in the rest of this paper, are

$$
\begin{array}{ll}
w=0, \text { scalars: } \quad f=f(X), \\
w=1, \text { vectors: } \quad V=V^{M}(X) Z_{M}, \\
w=2, \text { tensors: } \quad T=\frac{1}{2} T^{M N}(X) Z_{M} Z_{N}-\frac{1}{2}\left(\hat{T}^{M} Z_{M}\right)^{\prime} .
\end{array}
$$

(In oscillator language, these correspond to $1, a_{1}^{\dagger}$, and $\left(a_{1}^{\dagger}\right)^{2} \oplus a_{2}^{\dagger}$, respectively.)

We now examine identities for commutators that follow directly from consideration of contour integrals for operator products. The basic identity is that the commutator of an integrated operator (over all $\sigma$ for fixed $\tau$ ) with another operator equals the integral of the former over a contour enclosing the latter in the operator product:

$$
\left[\int A, B(1)\right]=\oint_{1} d 2 A(2) B(1),
$$

where $A$ and $B$ are arbitrary operators, expressed in terms of the currents $Z$ and functions of $X$ (fields). In the following sections we'll examine relevant special cases; for now we look at general properties.

The charge $\int A$ generates symmetry transformations $\delta_{A}$ on "covariant" operators $B$ as

$$
\delta_{A} B=\left[\int A, B\right],
$$

for symmetry parameters and fields appearing as functions in the operators $A$ and $B$, respectively. As always, symmetry transformations define a Lie derivative: in particular, in the case of quantum mechanics the representation of the Lie derivative/infinitesimal symmetry transformation on a field (denoted by $\delta_{A}$ ) can be obtained by an operator commutator (with the field represented by an operator $B$ ). Of course, the operator $B$ must 
contain enough terms so that these transformations close on the fields contained therein, and the operators $A$ must contain enough terms so that their algebra closes.

In the following sections we will evaluate operator products for the relevant fields. We will focus on two particular symmetries, to be analyzed in detail in the following sections:

1) When the symmetry parameter is an $O(D, D)$ vector, multiplying a single current $Z$, it describes "gauge" symmetries, specifically those that reduce to D-dimensional coordinate transformations and the gauge transformations of the 2-form field [10].

2) When the parameter is a symmetric second-rank $O(D, D)$ tensor, multiplying two currents $Z$, it describes worldsheet conformal (coordinate) transformations. It is then natural to multiply the second rank tensor by a single (scalar) world sheet parameter $\lambda(z)$.

\subsection{Bilinear operator products}

In this section we will introduce families of bilinear (quadratic) products of operators starting from the operator product expansion of two operators. Consider operators $\mathcal{O}_{1}$ and $\mathcal{O}_{2}$ of weights $w_{1}$ and $w_{2}$ respectively:

$$
w_{1}=\tilde{w}\left(\mathcal{O}_{1}\right), \quad w_{2}=\tilde{w}\left(\mathcal{O}_{2}\right)
$$

Their OPE is now written as

$$
\mathcal{O}_{1}(1) \mathcal{O}_{2}(2)=\sum_{w=-\infty}^{w_{1}+w_{2}} \frac{1}{z_{12}^{w}}\left(\mathcal{O}_{1} \circ_{w_{1}+w_{2}-w} \mathcal{O}_{2}\right)(2)
$$

The above expansion defines products $o_{w}$ with $w$ an integer greater than or equal to zero. The subscript on the product indicates the weight of the operator, independently of the weights of $\mathcal{O}_{1}$ and $\mathcal{O}_{2}$ :

$$
\tilde{w}\left(\mathcal{O}_{1} \circ_{w} \mathcal{O}_{2}\right)=w .
$$

Note that the expansion in conformal weight is associated with the change in power of $z$, as follows from Taylor expansion and the propagators of the previous section. We can write the above OPE as

$$
\begin{aligned}
\mathcal{O}_{1}(1) \mathcal{O}_{2}(2) & =\sum_{w=0}^{\infty} \frac{1}{\left(z_{12}\right)^{w_{1}+w_{2}-w}}\left(\mathcal{O}_{1} \circ_{w} \mathcal{O}_{2}\right)(2) \\
& =\frac{1}{\left(z_{12}\right)^{w_{1}+w_{2}}} \mathcal{O}_{1} \circ_{0} \mathcal{O}_{2}(2)+\frac{1}{\left(z_{12}\right)^{w_{1}+w_{2}-1}} \mathcal{O}_{1} \circ_{1} \mathcal{O}_{2}(2)+\ldots
\end{aligned}
$$

In practice, the explicit forms of all these products are evaluated by use of the free propagators introduced in the previous section, the various terms coming from the possible combinations and permutations of these propagators.

Of particular interest is the scalar product $\circ_{0}$ of weight zero, which we write as a bracket:

$$
\left\langle\mathcal{O}_{1} \mid \mathcal{O}_{2}\right\rangle \equiv \mathcal{O}_{1} \circ_{0} \mathcal{O}_{2}
$$


Note that this product is defined even when the operators have different weight. In an explicit computation, the leading term in $\alpha^{\prime}$ contracts as many indices on the fields as possible with $\eta$ 's, the rest with derivatives:

$$
\begin{aligned}
\mathcal{O} & =\frac{1}{w_{\mathcal{O}} !} \mathcal{O}^{M_{1} \ldots M_{w_{\mathcal{O}}}} Z_{M_{1}} \cdots Z_{M_{w_{\mathcal{O}}}}+\ldots \\
\Rightarrow\left\langle\mathcal{O}_{>} \mid \mathcal{O}_{<}\right\rangle & =\frac{1}{w_{<} !\left(w_{>}-w_{<}\right) !}\left(\mathcal{O}_{>}\right)^{M_{1} \ldots M_{w_{>}}} \partial_{M_{1}} \cdots \partial_{M_{w_{>}-w_{<}}}\left(\mathcal{O}_{<}\right)_{M_{w_{>}-w_{<}+1} \ldots M_{w_{>}}}+\ldots
\end{aligned}
$$

where $>$ and $<$ refer to the higher and lower weights.

The products satisfy a couple of useful identities associated with differentiation:

$$
\text { derivative: } \begin{aligned}
\mathcal{O}_{1}^{\prime} \circ_{w} \mathcal{O}_{2} & =\left(w-w_{1}-w_{2}\right) \mathcal{O}_{1} \circ_{w} \mathcal{O}_{2}, \\
\left(\mathcal{O}_{1} \circ_{w} \mathcal{O}_{2}\right)^{\prime} & =\mathcal{O}_{1}^{\prime} \circ_{w+1} \mathcal{O}_{2}+\mathcal{O}_{1} \circ_{w+1} \mathcal{O}_{2}^{\prime} .
\end{aligned}
$$

The first follows by differentiating (3.6) or (3.8) with respect to $z_{1}$ and recalling that $\tilde{w}\left(\mathcal{O}^{\prime}\right)=\tilde{w}(\mathcal{O})+1$. The second follows by differentiation with respect to $z_{2}$ and use of the first identity.

Since all our operators are Grassmann even we have the equality $\mathcal{O}_{1}(1) \mathcal{O}_{2}(2)=$ $\mathcal{O}_{2}(2) \mathcal{O}_{1}(1)$ of operator products, and therefore the products satisfy certain symmetry properties. For the weight zero product, it follows from (3.8) that

$$
\left\langle\mathcal{O}_{1} \mid \mathcal{O}_{2}\right\rangle=(-1)^{w_{1}+w_{2}}\left\langle\mathcal{O}_{2} \mid \mathcal{O}_{1}\right\rangle
$$

More systematically, we can compare OPE's about $z_{1}$ and about $z_{2}$ using Taylor expansion with the relation $z_{1}=z_{2}+z_{12}$. The result is that the symmetry property of the products takes the form

$$
\text { symmetry: } \quad \mathcal{O}_{2} \circ_{w} \mathcal{O}_{1}=(-1)^{w_{1}+w_{2}-w} e^{-\mathcal{L}_{\mathcal{O}_{1}} \circ_{w}} \mathcal{O}_{2}
$$

where we have defined a (linear) operator $\mathcal{L}$ that acts on products to give products:

$$
\mathcal{L}\left(\mathcal{O}_{1} \circ_{w} \mathcal{O}_{2}\right) \equiv\left(\mathcal{O}_{1} \circ_{w-1} \mathcal{O}_{2}\right)^{\prime}
$$

The right-hand side is indeed a sum of products because of the second derivative identity. One can then verify that the iterated action of this operator gives

$$
(\mathcal{L})^{w^{\prime}}\left(\mathcal{O}_{1} \circ_{w} \mathcal{O}_{2}\right) \equiv\left(\mathcal{O}_{1} \circ_{w-w^{\prime}} \mathcal{O}_{2}\right)^{\left(w^{\prime}\right)}, \quad \text { and } \quad \circ_{w}=0 \quad \text { for } w<0 .
$$

The superscript $\left(w^{\prime}\right)$ means $z$-differentiation $w^{\prime}$ times. We have, for example

$$
\mathcal{O}_{2} \circ_{2} \mathcal{O}_{1}=(-1)^{w_{1}+w_{2}}\left(\mathcal{O}_{1} \circ_{2} \mathcal{O}_{2}-\left(\mathcal{O}_{1} \circ_{1} \mathcal{O}_{2}\right)^{\prime}+\frac{1}{2}\left(\mathcal{O}_{1} \circ_{0} \mathcal{O}_{2}\right)^{\prime \prime}\right)
$$

We say that this product has exchange parity $(-1)^{w_{1}+w_{2}}$, up to $z$-derivatives.

For higher-weight products, it is useful to define truly symmetric products. This can be done by explicit symmetrization or antisymmetrization, as appropriate, and modified 
further by adding lower-weight products of the same exchange symmetry, acted by $z$ derivatives to raise the weight. Since $\circ_{1}$, like $\circ_{0}$, does not include lower-weight products of the same symmetry, their definitions are unambiguous:

$$
\begin{aligned}
\mathcal{O}_{1} \bullet_{0} \mathcal{O}_{2} & \equiv \mathcal{O}_{1} \circ_{0} \mathcal{O}_{2}=\left\langle\mathcal{O}_{1} \mid \mathcal{O}_{2}\right\rangle \\
\mathcal{O}_{1} \bullet_{1} \mathcal{O}_{2} & \equiv \frac{1}{2}\left[\mathcal{O}_{1} \circ_{1} \mathcal{O}_{2}-(-1)^{w_{1}+w_{2}} \mathcal{O}_{2} \circ_{1} \mathcal{O}_{1}\right]
\end{aligned}
$$

For the rest, several alternative possibilites suggest themselves:

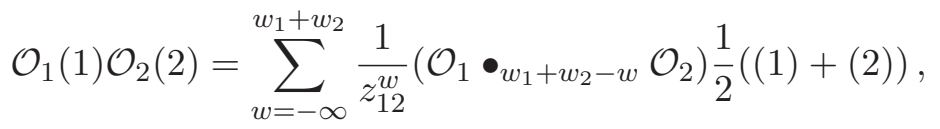

$$
\begin{aligned}
& \mathcal{O}_{1}(1) \mathcal{O}_{2}(2)=\sum_{w=-\infty}^{w_{1}+w_{2}} \frac{1}{z_{12}^{w}}\left(\mathcal{O}_{1} \bullet_{w_{1}+w_{2}-w} \mathcal{O}_{2}\right)\left(\frac{1}{2}\left(z_{1}+z_{2}\right)\right), \\
& \mathcal{O}_{1} \bullet{ }_{w} \mathcal{O}_{2} \equiv \frac{1}{2}\left[\mathcal{O}_{1} \circ{ }_{w} \mathcal{O}_{2}+(-1)^{w_{1}+w_{2}-w} \mathcal{O}_{2} \circ_{w} \mathcal{O}_{1}\right]
\end{aligned}
$$

where we use the notation

$$
\mathcal{O} \frac{1}{2}((1)+(2)) \equiv \frac{1}{2}\left(\mathcal{O}\left(z_{1}\right)+\mathcal{O}\left(z_{2}\right)\right) .
$$

All of these have definite exchange symmetry and satisfy a derivative identity

$$
\begin{aligned}
\mathcal{O}_{1} \bullet \bullet_{w} \mathcal{O}_{2} & =(-1)^{w_{1}+w_{2}-w} \mathcal{O}_{2} \bullet \mathcal{O}_{1} \\
\left(\mathcal{O}_{1} \bullet{ }_{w} \mathcal{O}_{2}\right)^{\prime} & =\mathcal{O}_{1}^{\prime} \bullet \bullet_{w+1} \mathcal{O}_{2}+\mathcal{O}_{1} \bullet{ }_{w+1} \mathcal{O}_{2}^{\prime} .
\end{aligned}
$$

Moreover, the three versions agree with the definitions of $\bullet_{0}$ and $\bullet_{1}$ in (3.17). The $\circ$ products can be expressed in terms of the $\bullet$ products as follows:

$$
\mathcal{O}_{1} \circ \mathcal{O}_{2}=\left[1+\tanh \left(\frac{1}{2} \mathcal{L}\right)\right] f(\mathcal{L}) \mathcal{O}_{1} \bullet \mathcal{O}_{2},
$$

where $f(\mathcal{L})=f(-\mathcal{L})$ and $f(0)=1$. The function $f$ takes the following forms for our three cases:

$$
\begin{aligned}
& \text { (1) } f(\mathcal{L})=\cosh ^{2} \frac{1}{2} \mathcal{L} \\
& \text { (2) } f(\mathcal{L})=\cosh \frac{1}{2} \mathcal{L} \\
& \text { (3) } f(\mathcal{L})=1,
\end{aligned}
$$

as easily verified by Taylor expansion about $z_{2}$. All these (anti)symmetrized products differ from the asymmetric ones only by total $z$-derivative terms, which play an auxiliary role. A particularly convenient choice of them will lead to a unique symmetric product, the star-product $\star$, defined with the help of the dilaton in section 6.2.

In the following we will make extensive use of the symmetry and derivative identities (3.13) and (3.11), usually without reference, except for a few early examples and some 
exceptional cases. This should be obvious: for any expression $A^{\prime} \circ B$ we use the derivative identity to remove the prime; for any expression where we wish to reorder a product we use the symmetry identity. For convenient reference, we have collected the most frequently used identities in the appendix.

The operator product expansion in terms of o products can be used to evaluate commutators, such as $\left[\int \lambda \mathcal{O}_{1}, \mathcal{O}_{2}\right]$. Here $\lambda(z)$ is a worldsheet parameter that depends on $z$, but not on $X(z)$, so it does not contribute propagators. We then find

$$
\left[\int \lambda \mathcal{O}_{1}, \mathcal{O}_{2}\right]=\sum_{w=1}^{w_{1}+w_{2}} \frac{1}{(w-1) !} \lambda^{(w-1)} \mathcal{O}_{1} \circ_{w_{1}+w_{2}-w} \mathcal{O}_{2}
$$

where the integration around the position $z_{2}$ of the second operator picks out just the singular part of the operator product. In here we used the integration identity

$$
\oint_{z_{2}} \frac{d z_{1}}{2 \pi i} \frac{1}{z_{12}^{n+1}} A\left(z_{1}\right)=\frac{1}{n !} A^{(n)}\left(z_{2}\right), \quad n \geq 0 .
$$

For example, we have

$$
\begin{aligned}
\oint_{z_{2}} \frac{d z_{1}}{2 \pi i} \frac{1}{z_{12}^{3}} 2 A\left(z_{1}\right) & =A^{\prime \prime}\left(z_{2}\right)=\left(Z^{M} \partial_{M} A\right)^{\prime}=\left(Z^{M}\right)^{\prime} \partial_{M} A+Z^{M}\left(\partial_{M} A\right)^{\prime} \\
& =Z^{M} \partial_{M} A+Z^{M} Z^{N} \partial_{N} \partial_{M} A
\end{aligned}
$$

\subsection{Cubic relations}

Although we have used operator product expansions in place of commutators, commutators are equivalent to just the singular parts of OPE's. However, OPE's of more than two operators can be unwieldy. In particular, Jacobi identities are easier than associativity identities, which require keeping finite terms after the first product, contributing to infinite sums.

Two important identities are the distributivity identity

$$
\text { distributivity: } \quad\left[\int A, B(1) C(2)\right]=\left[\int A, B(1)\right] C(2)+B(1)\left[\int A, C(2)\right] \text {, }
$$

which follows from

$$
\begin{aligned}
{\left[\int A, B(1) C(2)\right] } & =\oint_{1,2} d 3 A(3) B(1) C(2) \\
& =\oint_{1} d 3 A(3) B(1) C(2)+\oint_{2} d 3 A(3) B(1) C(2) \\
& =\left[\int A, B(1)\right] C(2)+B(1)\left[\int A, C(2)\right]
\end{aligned}
$$

and the Jacobi identity

$$
\text { Jacobi: }\left[\int A_{[1},\left[\int A_{2]}, B\right]\right]=\left[\left[\int A_{1}, \int A_{2}\right], B\right],
$$

which follows from distributivity upon integrating $B(1)$ about $z_{2}$. 
The distributivity identity proves the symmetry invariance of field equations, since we will derive the field equations through preservation of the Virasoro operator algebra. The Jacobi identity proves the closure of the symmetry transformations of the background fields we will introduce below:

$$
\left[\int A_{1}, \int A_{2}\right]=\int A_{12} \Rightarrow\left[\delta_{A_{1}}, \delta_{A_{2}}\right]=-\delta_{A_{12}} .
$$

The explicit action of $\delta_{A}$ on various fields, and the explicit form of $A_{12}$ in terms of $A_{1}$ and $A_{2}$, as evaluated by the above operator commutators, is a subject of the following sections.

We can derive various identities for these infinite classes of products by applying these identities, and expanding in powers of $z$, including the implicit ones now appearing as derivatives on $\lambda$. For the distributivity identity, which we write as

$$
\left[\int \lambda \mathcal{O}_{1}, \mathcal{O}_{2} \mathcal{O}_{3}\right]-\mathcal{O}_{2}\left[\int \lambda \mathcal{O}_{1}, \mathcal{O}_{3}\right]=\left[\int \lambda \mathcal{O}_{1}, \mathcal{O}_{2}\right] \mathcal{O}_{3}
$$

we find

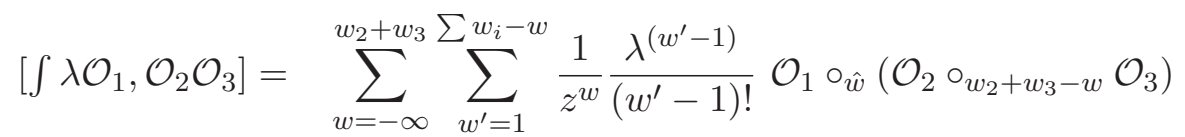

$$
\begin{aligned}
& \mathcal{O}_{2}\left[\int \lambda \mathcal{O}_{1}, \mathcal{O}_{3}\right]=\sum_{w^{\prime}=1}^{w_{1}+w_{3}} \sum_{w=-\infty}^{\sum w_{i}-w^{\prime}} \frac{1}{z^{w}} \frac{\lambda^{\left(w^{\prime}-1\right)}}{\left(w^{\prime}-1\right) !} \mathcal{O}_{2} \circ_{\hat{w}}\left(\mathcal{O}_{1} \circ_{w_{1}+w_{3}-w^{\prime}} \mathcal{O}_{3}\right) \\
& {\left[\int \lambda \mathcal{O}_{1}, \mathcal{O}_{2}\right] \mathcal{O}_{3}=\sum_{w^{\prime \prime}=0}^{\infty} \sum_{w^{\prime}=w^{\prime \prime}+1}^{w_{1}+w_{2}+w^{\prime \prime}}\left(\begin{array}{c}
w^{\prime}-1 \\
w^{\prime \prime}
\end{array}\right) \sum_{w=-\infty}^{\sum w_{i}-w^{\prime}} \frac{1}{z^{w}} \frac{\lambda^{\left(w^{\prime}-1\right)}}{\left(w^{\prime}-1\right) !}} \\
& \times\left(\mathcal{O}_{1} \circ_{w_{1}+w_{2}+w^{\prime \prime}-w^{\prime}} \mathcal{O}_{2}\right) \circ_{\hat{w}} \mathcal{O}_{3},
\end{aligned}
$$

where $z=z_{23}$, all operators and $\lambda$ 's are evaluated at $z_{3}$, and

$$
\hat{w} \equiv \sum w_{i}-w-w^{\prime}, \quad \sum w_{i}=w_{1}+w_{2}+w_{3} .
$$

(For the last line in (3.31) the third sum arises because we need to Taylor expand $\lambda(2)$ about $z_{3}$.)

We then compare terms of fixed order $w$ and $w^{\prime}$ in derivatives of $\lambda$ and powers of $z$. Paying attention to the limits of summation we find

$$
\begin{array}{r}
\mathcal{O}_{1} \circ_{\hat{w}}\left(\mathcal{O}_{2} \circ_{w_{2}+w_{3}-w} \mathcal{O}_{3}\right)-\mathcal{O}_{2} \circ_{\hat{w}}\left(\mathcal{O}_{1} \circ_{w_{1}+w_{3}-w^{\prime}} \mathcal{O}_{3}\right) \\
=\sum_{w^{\prime \prime}=1}^{w^{\prime}}\left(\begin{array}{c}
w^{\prime}-1 \\
w^{\prime \prime}-1
\end{array}\right)\left(\mathcal{O}_{1} \circ_{w_{1}+w_{2}-w^{\prime \prime}} \mathcal{O}_{2}\right) \circ_{\hat{w}} \mathcal{O}_{3}
\end{array}
$$

where always

$$
-\infty \leq w+w^{\prime} \leq \sum w_{i}, \quad 1 \leq w^{\prime},
$$

which means that we get identities for any $w$ satisfying

$$
w \leq-1+\sum w_{i}=w_{\max }
$$


We get one identity for $w=w_{\max }$, two identities for $w=w_{\max }-1$, three identities for $w=w_{\max }-2$ and so forth and so on.

For Jacobi, we examine

$$
\left[\int \lambda_{1} \mathcal{O}_{1},\left[\int \lambda_{2} \mathcal{O}_{2}, \mathcal{O}_{3}\right]\right]-\left[\int \lambda_{2} \mathcal{O}_{2},\left[\int \lambda_{1} \mathcal{O}_{1}, \mathcal{O}_{3}\right]\right]=\left[\left[\int \lambda_{1} \mathcal{O}_{1}, \int \lambda_{2} \mathcal{O}_{2}\right], \mathcal{O}_{3}\right]
$$

The manipulations and results are almost the same as for distributivity, only now only singular terms contribute, so

$$
1 \leq w, 1 \leq w^{\prime}
$$

and things are antisymmetric in $w$ and $w^{\prime}$ (when 1's and 2's are switched). The result is the same as for distributivity, except for the restriction on the lower limit of $w$ to singular terms.

\section{Vector gauge symmetry}

In this section we will examine the quantum corrections to generalized brackets and (Lie) derivatives introduced in previous papers for the gauge symmetries. We begin by considering general properties of current algebra that should generalize to other string models. For the vector operator

$$
\Xi(z)=\xi^{M}(X(z)) Z_{M}(z),
$$

with gauge parameter $\xi^{M}(X)$, we define the gauge transformation $\delta_{\xi} B$ of the arbitrary operator $B$ of weight $\tilde{w}(B)$ by

$$
\delta_{\xi} B \equiv\left[\int \Xi, B\right]=\Xi \circ_{B} B, \quad \circ_{B} \equiv \circ_{\tilde{w}(B)} .
$$

The equality after the definition follows by using (3.6) for $\Xi$ and $B$ :

$$
\Xi(1) B(2)=\text { regular }+\frac{1}{z_{12}}\left(\Xi \circ_{B} B\right)(2)+\frac{1}{z_{12}^{2}}\left(\Xi \circ_{B-1} B\right)(2)+\ldots
$$

The gauge transformation $\delta_{\xi}$ vanishes if the gauge parameter is "gauge for gauge", $\xi^{M}=$ $\partial^{M} \zeta$, since

$$
\int \Xi=\int Z^{M} \partial_{M} \zeta=\int \zeta^{\prime}=0 .
$$

It is a fundamental property that all products $\circ$ are gauge covariant:

$$
\delta_{\xi}\left(A \circ_{w} B\right)=\left(\delta_{\xi} A\right) \circ_{w} B+A \circ_{w}\left(\delta_{\xi} B\right) .
$$

This follows from the distributive identity

$$
\left[\int \Xi, A(1) B(2)\right]=\left[\int \Xi, A\right](1) B(2)+A(1)\left[\int \Xi, B\right](2),
$$

and use of (3.6) for each term to find

$$
\left[\int \Xi, A \circ_{w} B\right]=\left[\int \Xi, A\right] \circ_{w} B+A \circ_{w}\left[\int \Xi, B\right],
$$

which is equivalent to (4.5). Taking a $z$-derivative is also a covariant operation,

$$
\delta_{\xi}\left(A^{\prime}\right)=\left(\delta_{\xi} A\right)^{\prime},
$$


as we check using the derivative identities:

$$
\left(\Xi \circ_{A} A\right)^{\prime}=\Xi^{\prime} \circ_{A+1} A+\Xi \circ_{A+1} A^{\prime}=0+\Xi \circ_{A^{\prime}} A^{\prime} .
$$

All versions of $\bullet$ products are also gauge covariant: they are built from $\circ$ products and $z$-derivatives of $\circ$ products. So is the $\mathcal{L}$ operator, in the sense that $\delta_{\xi} \mathcal{L}=\mathcal{L} \delta_{\xi}$ holds when acting on (sums of) bilinear products of operators.

In the following we will discuss the operator product of currents that give us inner brackets, $\mathrm{C}$ and $\mathrm{D}$ brackets, and outer products, all of them with $\alpha^{\prime}$ corrections. They will be evaluated explicitly, and the $\mathrm{C}$ bracket Jacobiator will be shown to be a trivial vector. We also evaluate the gauge transformations of scalars, vectors, and tensors.

\subsection{Inner and outer products, brackets}

The key ingredients for the theory we are to construct arise in a simple manner from the OPE expansion $\Xi_{1}(1) \Xi_{2}(2)$ of two currents $\Xi_{1}=\xi_{1}^{M} Z_{M}$ and $\Xi_{2}=\xi_{2}^{M} Z_{M}$. Indeed, the operators in this expansion define the inner product, the various brackets, and a set of useful products.

The brackets in generalized geometry come in Courant and Dorfman varieties. Their double field theory versions, without $\alpha^{\prime}$ corrections, are the $\mathrm{C}$ bracket of [11] and the D-bracket [17]. The $\mathrm{C}$ bracket when restricted from $\mathrm{D}+\mathrm{D}$ dimensions to $\mathrm{D}$ dimensions becomes the Courant bracket [17]. Similarly, upon reduction, the D bracket becomes the Dorfman bracket.

The $\mathrm{C}$ and $\mathrm{D}$ varieties of brackets arise by doing the OPE of two currents in slightly different ways. In the $\mathrm{C}$ case the normal ordered operators are averaged over the two points, while in the $\mathrm{D}$ case the operators are located at the position of the second current. In the following, the "quantum" contributions to the OPE give the $\alpha^{\prime}$ corrected brackets, as well as corrected inner products and other products. We call these the new brackets and products. Upon reduction to D dimensions they give new versions of the Courant and Dorfman brackets, as well as a new inner product.

We thus have two forms of the OPE:

$$
\begin{aligned}
& \Xi_{1}(1) \Xi_{2}(2) \equiv\left[\frac{1}{z_{12}^{2}}\left\langle\Xi_{1} \mid \Xi_{2}\right\rangle+\frac{1}{z_{12}}\left[\Xi_{1}, \Xi_{2}\right]_{C}+: \Xi_{1} \Xi_{2}:_{C}\right] \frac{1}{2}((1)+(2))+\mathcal{O}\left(z_{12}\right), \\
& \Xi_{1}(1) \Xi_{2}(2) \equiv\left[\frac{1}{z_{12}^{2}}\left\langle\Xi_{1} \mid \Xi_{2}\right\rangle+\frac{1}{z_{12}}\left[\Xi_{1}, \Xi_{2}\right]_{D}+: \Xi_{1} \Xi_{2}:_{D}\right](2)+\mathcal{O}\left(z_{12}\right) .
\end{aligned}
$$

In our previous notation, we thus have

$$
\left[V_{1}, V_{2}\right]_{D} \equiv V_{1} \circ_{1} V_{2}, \quad\left[V_{1}, V_{2}\right]_{C} \equiv V_{1} \bullet_{1} V_{2}, \quad: V_{1} V_{2}:_{D} \equiv V_{1} \circ_{2} V_{2}, \quad: V_{1} V_{2}:_{C} \equiv V_{1} \bullet_{2} V_{2},
$$

as well as the previously defined $\langle\mid\rangle \equiv \circ_{0}=\bullet_{0}$, where we have made a particular choice of the ambiguous $\bullet_{2}$.

The two above expansions are simply related by

$$
A \frac{1}{2}((1)+(2))=\left(A+\frac{1}{2} z_{12} A^{\prime}+\frac{1}{4} z_{12}^{2} A^{\prime \prime}\right)(2)+\mathcal{O}\left(z_{12}^{3}\right)
$$


and thus the inner product, at the second-order pole, is the same for the two, while the other terms are related by

$$
\begin{aligned}
& {\left[\Xi_{1}, \Xi_{2}\right]_{D}=\left[\Xi_{1}, \Xi_{2}\right]_{C}+\frac{1}{2}\left\langle\Xi_{1} \mid \Xi_{2}\right\rangle^{\prime}} \\
& : \Xi_{1} \Xi_{2}:_{D}=: \Xi_{1} \Xi_{2}:_{C}+\frac{1}{2}\left[\Xi_{1}, \Xi_{2}\right]_{D}^{\prime} .
\end{aligned}
$$

The $\mathrm{C}$ form is more useful for symmetry: clearly

$$
\left\langle\Xi_{1} \mid \Xi_{2}\right\rangle=\left\langle\Xi_{2} \mid \Xi_{1}\right\rangle, \quad\left[\Xi_{1}, \Xi_{2}\right]_{C}=-\left[\Xi_{2}, \Xi_{1}\right]_{C}, \quad: \Xi_{1} \Xi_{2}:_{C}=: \Xi_{2} \Xi_{1}:_{C} .
$$

The $\mathrm{D}$ bracket, as opposed to the $\mathrm{C}$ bracket, is not antisymmetric in its inputs. One readily sees that the $\mathrm{C}$ bracket is obtained by antisymmetrization of the $\mathrm{D}$ bracket

$$
\left[\Xi_{1}, \Xi_{2}\right]_{C} \equiv \frac{1}{2}\left[\Xi_{[1}, \Xi_{2]}\right]_{D}
$$

The brackets can be also viewed as current algebra commutators. For example, consider the single commutator $\left[\int \Xi_{1}, \Xi_{2}\right]$. We can use the OPE in (4.10) to see that this selects the D bracket

$$
\left[\int \Xi_{1}, \Xi_{2}\right]=\left[\Xi_{1}, \Xi_{2}\right]_{D}=\left[\Xi_{1}, \Xi_{2}\right]_{D}^{M} Z_{M}
$$

Clearly the D-bracket then defines a distributive "D-derivative",

$$
\left[\int \Xi_{3}, \Xi_{1}(1) \Xi_{2}(2)\right]=\left[\int \Xi_{3}, \Xi_{1}(1)\right] \Xi_{2}(2)+\Xi_{1}(1)\left[\int \Xi_{3}, \Xi_{2}(2)\right]
$$

as follows from the distributivity identity of the previous subsection. This is a special case of the distributivity of the Lie derivative/gauge transformation $\delta_{\xi}$.

Of course, the algebra of integrated currents, and thus gauge transformations, closes. We can now express this algebra in terms of the new brackets: from (4.16)

$$
\left[\int \Xi_{1}, \int \Xi_{2}\right]=\int\left[\Xi_{1}, \Xi_{2}\right]_{D}=\int\left[\Xi_{1}, \Xi_{2}\right]_{C},
$$

using the fact that the 2 brackets differ only by a total derivative. We can thus identify

$$
\left[\int \Xi_{1}, \int \Xi_{2}\right]=\int \Xi_{12} \quad \Rightarrow \quad \Xi_{12}=\left[\Xi_{1}, \Xi_{2}\right]_{C}
$$

without loss of generality, so that $\Xi_{12}$ preserves the antisymmetry of $\int \Xi_{12}$. This defines the algebra of gauge transformations:

$$
\left[\delta_{\xi_{1}}, \delta_{\xi_{2}}\right]=-\left[\delta_{\xi_{2}}, \delta_{\xi_{1}}\right]=-\delta_{\xi_{12}}, \quad \xi_{12}^{M}=\left[\Xi_{1}, \Xi_{2}\right]_{C}^{M}
$$

All these objects will be computed explicitly in the following subsection. 


\subsection{Evaluation}

We now evaluate the OPE of two currents for the theory under consideration. The possible contractions give:

$$
\begin{aligned}
\Xi_{1}(1) \Xi_{2}(2)=: \xi_{1}^{M} & (1) Z_{M}(1) \xi_{2}^{N}(2) Z_{N}(2) \\
& +\xi_{1}^{M}(1)\left\langle Z_{M}(1) \xi_{2}^{N}(2)\right\rangle Z_{N}(2)+\xi_{2}^{M}(2)\left\langle Z_{M}(2) \xi_{1}^{N}(1)\right\rangle Z_{N}(1) \\
& +\xi_{1}^{M}(1) \xi_{2}^{N}(2)\left\langle Z_{M}(1) Z_{N}(2)\right\rangle+\left\langle Z_{M}(2) \xi_{1}^{N}(1)\right\rangle\left\langle Z_{N}(1) \xi_{2}^{M}(2)\right\rangle:
\end{aligned}
$$

The last term, with a double contraction, is the quantum correction. Using (2.9) to evaluate the above contractions we find

$$
\begin{aligned}
\Xi_{1}(1) \Xi_{2}(2)=:\left(\xi_{1}^{M}\right. & \left.Z_{M}\right)(1)\left(\xi_{2}^{N} Z_{N}\right)(2) \\
& +\frac{1}{z_{12}}\left(\xi_{1}^{M}(1)\left(\partial_{M} \xi_{2}^{N} Z_{N}\right)(2)-\xi_{2}^{M}(2)\left(\partial_{M} \xi_{1}^{N} Z_{N}\right)(1)\right) \\
& +\frac{1}{z_{12}^{2}}\left(\xi_{1}^{M}(1) \xi_{2 M}(2)-\partial_{M} \xi_{1}^{N}(1) \partial_{N} \xi_{2}^{M}(2)\right): .
\end{aligned}
$$

As we will see, the second line will contribute to the usual Lie bracket/commutator. A contribution from the first term on the third line modifies it to the classical C- or D-bracket. A contribution from the last term gives the quantum correction. We will use the following expansion of a normal-ordered product of operators

$$
A\left(z_{1}\right) B\left(z_{2}\right)=\left[A B-\frac{1}{2} z_{12} A \overleftrightarrow{\partial} B-\frac{1}{2} z_{12}^{2} A^{\prime} B^{\prime}+\mathcal{O}\left(z_{12}^{3}\right)\right] \frac{1}{2}((1)+(2))
$$

(As usual, normal ordering is assumed for operators evaluated at the same point.) Here the $z$ derivative $\stackrel{\leftrightarrow}{\partial}$ is defined to act as $A \stackrel{\leftrightarrow}{\partial} B \equiv A B^{\prime}-A^{\prime} B$. We now use this equation to expand the right-hand side of (4.22) and we obtain a result that can be put in the form of the top equation in (4.10).

The residue of the second order pole defines a new symmetric inner product given by

$$
\text { Inner product: }\left\langle\Xi_{1} \mid \Xi_{2}\right\rangle=\xi_{1}^{M} \xi_{2}^{N} \eta_{M N}-\left(\partial_{N} \xi_{1}^{M}\right)\left(\partial_{M} \xi_{2}^{N}\right) \text {. }
$$

The first term is the familiar one and the second is the $\alpha^{\prime}$ correction, arising from a quantum contribution in the OPE. This correction vanishes if any of the $\xi$ 's is trivial $\left(\xi^{M}=\partial^{M} \chi\right)$ and the whole inner product vanishes if both $\xi$ 's are trivial. Equivalently, $\left\langle A^{\prime} \mid B^{\prime}\right\rangle=0$, recalling that $\left(A^{\prime}\right)^{M}=\partial^{M} A$. Using the strong constraint, the new inner product can also be written as

$$
\left\langle\Xi_{1} \mid \Xi_{2}\right\rangle=\xi_{1}^{M} \xi_{2}^{N} \eta_{M N}+\frac{1}{2} K_{1}^{M N} K_{2 M N},
$$

where $K_{M N}$ is the "field strength" of the gauge parameter:

$$
K_{M N} \equiv \partial_{[M} \xi_{N]} \equiv \partial_{M} \xi_{N}-\partial_{N} \xi_{M}
$$

Reducing to $\mathrm{D}$ dimensions by setting $\tilde{\partial}^{i}$ derivatives to zero gives, with $\left(\tilde{\xi}_{1 i}, \xi_{1}^{i}\right)$ and $\left(\tilde{\xi}_{2 i}, \xi_{2}^{i}\right)$ the one-form and vector components of $\xi_{1}^{M}$ and $\xi_{2}^{M}$, respectively:

$$
\left\langle\Xi_{1} \mid \Xi_{2}\right\rangle=\xi_{1}^{i} \tilde{\xi}_{2 i}+\xi_{2}^{i} \tilde{\xi}_{1 i}-\partial_{i} \xi_{1}^{j} \partial_{j} \xi_{2}^{i}
$$

The last term is the quantum correction. 
The first-order pole contains the corrected C-bracket, a skew bracket that we write compactly as:

$$
\left[\Xi_{1}, \Xi_{2}\right]_{C}=\left[\Xi_{1}, \Xi_{2}\right]_{L}-\frac{1}{2}\left\langle\Xi_{1}|\overleftrightarrow{\partial}| \Xi_{2}\right\rangle
$$

Here $\stackrel{\leftrightarrow}{\partial}$ translates as $\partial=Z^{M} \partial_{M}$, the bracket $[,]_{L}$ is the commutator/Lie bracket

$$
\left[\Xi_{1}, \Xi_{2}\right]_{L} \equiv\left(\xi_{[1}^{N} \partial_{N} \xi_{2]}^{M}\right) Z_{M}
$$

and the correction to the C-bracket is produced by the correction of the inner product. More explicitly the above formula reads

C bracket: $\left[\Xi_{1}, \Xi_{2}\right]_{C}^{M}=\xi_{[1}^{N} \partial_{N} \xi_{2]}^{M}-\frac{1}{2} \xi_{1}^{K} \stackrel{\leftrightarrow}{\partial}{ }^{M} \xi_{2 K}+\frac{1}{2}\left(\partial_{K} \xi_{1}^{L}\right) \stackrel{\leftrightarrow}{\partial}{ }^{M}\left(\partial_{L} \xi_{2}^{K}\right)$.

The last term, with three derivatives, is the new correction.

Upon reduction from $\mathrm{D}+\mathrm{D}$ to $\mathrm{D}$ dimensions the vector part of the bracket is not corrected, but the one-form part is

$$
\begin{aligned}
& \left(\left[\Xi_{1}, \Xi_{2}\right]_{C}\right)^{i}=\xi_{[1}^{k} \partial_{k} \xi_{2]}^{i}, \\
& \left(\left[\Xi_{1}, \Xi_{2}\right]_{C}\right)_{i}=\xi_{[1}^{k} \partial_{k} \tilde{\xi}_{2] i}+\frac{1}{2}\left(\xi_{1}^{k} \overleftrightarrow{\leftrightarrow}_{i} \tilde{\xi}_{2 k}+\tilde{\xi}_{1 k} \overleftrightarrow{\leftrightarrow}_{i} \xi_{2}^{k}\right)+\frac{1}{2}\left(\partial_{k} \xi_{1}^{\ell}\right) \overleftrightarrow{\partial}_{i}\left(\partial_{\ell} \xi_{2}^{k}\right) .
\end{aligned}
$$

The last term with three derivatives is the quantum correction.

Finally, the regular term in the OPE defines a tensor operator of weight two. The two-index part defines an outer ("star" *) product constructed from the two $\xi$ 's. The oneindex part defines a product (b), built from the two $\xi$ 's as well. As will be explained in the next section, the two-index part defines a tensor by itself, but the one-index part does not. Thus the (b) product, which enters the tensor as total derivative, is less interesting. We have

$$
: \Xi_{1} \Xi_{2}:_{C}=\Xi_{1} * \Xi_{2}-\left(\Xi_{1} b \Xi_{2}\right)_{C}^{\prime} .
$$

Both products are symmetric, a property they inherit from the OPE,

$$
\Xi_{1} * \Xi_{2}=\Xi_{2} * \Xi_{1}, \quad\left(\Xi_{1} b \Xi_{2}\right)_{C}=\left(\Xi_{2} b \Xi_{1}\right)_{C} .
$$

Explicitly,

Outer product: $\Xi_{1} * \Xi_{2} \equiv \frac{1}{2}\left[\xi_{(1}^{M} \xi_{2)}^{N}+\partial^{P} \xi_{(1}^{(M} \partial^{N)} \xi_{2) P}-\frac{1}{2} \partial^{M} \xi_{(1}^{P} \partial^{N} \xi_{2) P}\right.$

$$
\left.+\frac{1}{2} \partial^{M} \partial^{P} \xi_{(1}^{Q} \partial^{N} \partial_{Q} \xi_{2) P}\right] Z_{M} Z_{N} .
$$

We also give the b-product for completeness:

$$
\left(\Xi_{1} b \Xi_{2}\right)_{C} \equiv \frac{1}{2} \xi_{(1}^{K} \partial_{K} \xi_{2)}^{M} Z_{M}
$$

Being bilinear and symmetric, these two products (as well as the inner product) can be written in terms of squares. For example,

$$
\Xi_{1} * \Xi_{2}=\frac{1}{2}\left[\left(\Xi_{1}+\Xi_{2}\right) *\left(\Xi_{1}+\Xi_{2}\right)-\Xi_{1} * \Xi_{1}-\Xi_{2} * \Xi_{2}\right]
$$


so we need only define that:

$$
\Xi * \Xi=\left[\xi^{M} \xi^{N}+\partial^{P} \xi^{(M} \partial^{N)} \xi_{P}-\frac{1}{2} \partial^{M} \xi^{P} \partial^{N} \xi_{P}+\frac{1}{2} \partial^{M} \partial^{P} \xi^{Q} \partial^{N} \partial_{Q} \xi_{P}\right] Z_{M} Z_{N} .
$$

We now turn to the $\mathrm{D}$ form of the OPE. Using the relation to the $\mathrm{C}$ form,

$$
\left[\Xi_{1}, \Xi_{2}\right]_{D}=\left[\Xi_{1}, \Xi_{2}\right]_{C}+\frac{1}{2}\left\langle\Xi_{1} \mid \Xi_{2}\right\rangle^{\prime}=\left[\Xi_{1}, \Xi_{2}\right]_{L}+\left\langle\Xi_{2}|\partial| \Xi_{1}\right\rangle
$$

In components,

$$
\text { D bracket: }\left[\Xi_{1}, \Xi_{2}\right]_{D}^{M}=\xi_{[1}^{K} \partial_{K} \xi_{2]}^{M}+\partial^{M} \xi_{1}^{K} \xi_{2 K}-\partial^{M} \partial_{K} \xi_{1}^{L} \partial_{L} \xi_{2}^{K} .
$$

Since the extra term in : $\Xi_{1} \Xi_{2}:_{D}$ is a total derivative, " $\star$ " is unchanged, but we have a different bilinear, symmetric, auxiliary product:

$$
: \Xi_{1} \Xi_{2}:_{D} \equiv \Xi_{1} * \Xi_{2}-\left(\Xi_{1} b \Xi_{2}\right)_{D}^{\prime}
$$

where

$$
\left(\Xi_{1} b \Xi_{2}\right)_{D}=\left(\Xi_{1} b \Xi_{2}\right)_{C}-\frac{1}{2}\left[\Xi_{1}, \Xi_{2}\right]_{D}
$$

In components

$$
\left(\Xi_{1} b \Xi_{2}\right)_{D} \equiv\left[\xi_{2}^{K} \partial_{K} \xi_{1}^{M}-\frac{1}{2} \partial^{M} \xi_{1}^{K} \xi_{2 K}+\frac{1}{2} \partial^{M} \partial_{K} \xi_{1}^{L} \partial_{L} \xi_{2}^{K}\right] Z_{M}
$$

As for any gauge transformation, one can view the $\mathrm{D}$ bracket as defining a (generalized) Lie derivative. For a current $V=V^{M} Z_{M}$

$$
\mathbf{L}_{\xi} V \equiv[\Xi, V]_{D} \quad \rightarrow \quad \mathbf{L}_{\xi} V^{M}=\xi^{P} \partial_{P} V^{M}+\left(\partial^{M} \xi_{P}-\partial_{P} \xi^{M}\right) V^{P}-\partial^{M} \partial_{K} \xi^{L} \partial_{L} V^{K}
$$

where the last term is the $\alpha^{\prime}$ correction to the generalized Lie derivative introduced in [11]. Upon reduction to $\mathrm{D}$ dimensions, the Lie derivative of a vector receives no correction but the Lie derivative of a one-form does

$$
\begin{aligned}
& \left(\mathbf{L}_{\xi} V\right)^{i}=\xi^{k} \partial_{k} V^{i}-V^{k} \partial_{k} \xi^{i} \\
& \left(\mathbf{L}_{\xi} V\right)_{i}=\xi^{k} \partial_{k} V_{i}+\partial_{i} \xi^{p} V_{p}+\left(\partial_{i} \tilde{\xi}_{p}-\partial_{p} \tilde{\xi}_{i}\right) V^{p}-\partial_{i} \partial_{k} \xi^{p} \partial_{p} V^{k} .
\end{aligned}
$$

The last term on the second line is the correction.

\subsection{Jacobiator and N-tensor}

The C-bracket, while antisymmetric, is not a Lie bracket, since it does not satisfy a Jacobi identity. The failure to satisfy a Jacobi identity is measured by the Jacobiator $J_{C}\left(\Xi_{1}, \Xi_{2}, \Xi_{3}\right)$ defined by

$$
\begin{aligned}
J_{C}\left(\Xi_{1}, \Xi_{2}, \Xi_{3}\right) & \equiv\left[\left[\Xi_{1}, \Xi_{2}\right]_{C}, \Xi_{3}\right]_{C}+\left[\left[\Xi_{2}, \Xi_{3}\right]_{C}, \Xi_{1}\right]_{C}+\left[\left[\Xi_{3}, \Xi_{1}\right]_{C}, \Xi_{2}\right]_{C} \\
& =-\left(\left[\Xi_{1},\left[\Xi_{2}, \Xi_{3}\right]_{C}\right]_{C}+\left[\Xi_{2},\left[\Xi_{3}, \Xi_{1}\right]_{C}\right]_{C}+\left[\Xi_{3},\left[\Xi_{1}, \Xi_{2}\right]_{C}\right]_{C}\right) \\
& =-\frac{1}{2}\left[\Xi_{[1},\left[\Xi_{2}, \Xi_{3}\right]_{C}\right]_{C}
\end{aligned}
$$


where the antisymmetrization on the last line is over the three indices, making the Jacobiator manifestly antisymmetric on the three currents $\Xi_{1}, \Xi_{2}, \Xi_{3}$. In this section we calculate this Jacobiator. As it turns out, the above C-Jacobiator is actually proportional to the D-Jacobiator, defined by

$$
J_{D}\left(\Xi_{1}, \Xi_{2}, \Xi_{3}\right) \equiv-\frac{1}{2}\left[\Xi_{[1},\left[\Xi_{2}, \Xi_{3}\right]_{D}\right]_{D} .
$$

While the $\mathrm{D}$ bracket is not antisymmetric, the above Jacobiator is.

To motivate the answer for this calculation let us consider the rewriting:

$$
\left[\Xi_{[1},\left[\Xi_{2}, \Xi_{3]}\right]_{D}\right]_{D}=\left[\int \Xi_{[1},\left[\int \Xi_{2}, \Xi_{3]}\right]\right] .
$$

The right-hand side is a current that when integrated must give zero since $\left[\int \Xi_{[1},\left[\int \Xi_{2}, \int \Xi_{3]}\right]\right]=0$ trivially. Therefore this current must be a total derivative of a scalar $N$ built from the three currents

$$
\left[\Xi_{[1},\left[\Xi_{2}, \Xi_{3]}\right]_{D}\right]_{D}=4 N^{\prime}=4 Z^{M} \partial_{M} N
$$

where the coefficient was adjusted for later convenience. Note that the nontriviality of the Jacobiator does not imply the violation of the usual type of Jacobi identities for operator commutators, where the same operators appear in all terms, in contrast to the right-hand side of (4.47), where the choice of currents to be integrated varies from term to term.

$J_{C}$ and $J_{D}$ can be calculated conveniently at the same time. We first relate $J_{C}$ to $J_{D}$. Using twice the fact that the C-bracket is the antisymmetric part of the D-bracket (see (4.15)), we find:

$$
\left[\Xi_{[1},\left[\Xi_{2}, \Xi_{3]}\right]_{C}\right]_{C}=\left[\Xi_{[1},\left[\Xi_{2}, \Xi_{3]}\right]_{D}\right]_{C}=\frac{1}{2}\left(\left[\Xi_{[1},\left[\Xi_{2}, \Xi_{3]}\right]_{D}\right]_{D}-\left[\left[\Xi_{[1}, \Xi_{2}\right]_{D}, \Xi_{3]}\right]_{D}\right) .
$$

Then using the distributivity (4.17) of the D-bracket

$$
\begin{aligned}
& {\left[\Xi_{[1},\left[\Xi_{2}, \Xi_{3]}\right]_{D}\right]_{D}=\left[\left[\Xi_{[1}, \Xi_{2}\right]_{D}, \Xi_{3]}\right]_{D}+\left[\Xi_{[2},\left[\Xi_{1}, \Xi_{3]}\right]_{D}\right]_{D} } \\
\Rightarrow & {\left[\left[\Xi_{[1}, \Xi_{2}\right]_{D}, \Xi_{3]}\right]_{D}=2\left[\Xi_{[1},\left[\Xi_{2}, \Xi_{3]}\right]_{D}\right]_{D}, }
\end{aligned}
$$

a curious relation, since the $(+2)$ would be replaced by a $(-1)$ for an antisymmetric bracket (it shows that similar looking definitions of the D Jacobiator can be quite different). Back in (4.49) we find the anticipated relation between Jacobiators

$$
\left[\Xi_{[1},\left[\Xi_{2}, \Xi_{3}\right]_{C}\right]_{C}=-\frac{1}{2}\left[\Xi_{[1},\left[\Xi_{2}, \Xi_{3]}\right]_{D}\right]_{D} \Rightarrow J_{C}=-\frac{1}{2} J_{D} .
$$

We then again express the C-Jacobiator in terms of the D-Jacobiator using (4.15) for the inner C-bracket and the first of (4.13) for the outer C bracket:

$$
\left[\Xi_{[1},\left[\Xi_{2}, \Xi_{3]}\right]_{C}\right]_{C}=\left[\Xi_{[1},\left[\Xi_{2}, \Xi_{3]}\right]_{D}\right]_{C}=\left[\Xi_{[1},\left[\Xi_{2}, \Xi_{3]}\right]_{D}\right]_{D}-\frac{1}{2}\left\langle\Xi_{[1} \mid\left[\Xi_{2}, \Xi_{3]}\right]_{D}\right\rangle^{\prime} .
$$

Again, using (4.15) we can replace the $\mathrm{D}$ by a $\mathrm{C}$ inside the inner product, so that we have found

$$
-2 J_{C}=-2 J_{D}-\frac{1}{2}\left\langle\Xi_{[1} \mid\left[\Xi_{2}, \Xi_{3]}\right]_{C}\right\rangle^{\prime} \Rightarrow J_{C}=J_{D}+\frac{1}{4}\left\langle\left[\Xi_{[1}, \Xi_{2}\right]_{C} \mid \Xi_{3]}\right\rangle^{\prime} .
$$


It follows from this equation and (4.51) that

$$
J_{C}\left(\Xi_{1}, \Xi_{2}, \Xi_{3}\right)=\frac{1}{12}\left\langle\left[\Xi_{[1}, \Xi_{2}\right]_{C} \mid \Xi_{3]}\right\rangle^{\prime}=N^{\prime},
$$

where $N$ can be written as

$$
N\left(\Xi_{1}, \Xi_{2}, \Xi_{3}\right)=\frac{1}{6}\left(\left\langle\left[\Xi_{1}, \Xi_{2}\right]_{C} \mid \Xi_{3}\right\rangle+\left\langle\left[\Xi_{2}, \Xi_{3}\right]_{C} \mid \Xi_{1}\right\rangle+\left\langle\left[\Xi_{3}, \Xi_{1}\right]_{C} \mid \Xi_{2}\right\rangle\right) .
$$

This result takes exactly the same form as that classical C-bracket Jacobiator [17], the only change is that now we use the $\alpha^{\prime}$ corrected brackets and inner product. More explicitly,

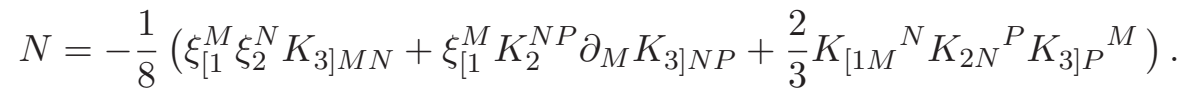

Note also that $J_{D}=-2 N^{\prime}$ and that is consistent with (4.48). The N-tensor was introduced in [11] as a field strength. In D dimensions, it reduces to the Nijenhuis tensor, that appears in the computation of the Jacobiator for the Courant bracket.

\subsection{Gauge transformations}

We have already seen examples of the three different kinds of covariant operators listed in (3.2): scalars, vectors, and tensors. The gauge transformations of the first two have already been treated:

$$
\begin{aligned}
\delta_{\xi} f & =\xi^{M} \partial_{M} f, \\
\delta_{\xi} V^{M} & \equiv[\Xi, V]_{D}^{M}=\xi^{P} \partial_{P} V^{M}+\left(\partial^{M} \xi_{P}-\partial_{P} \xi^{M}\right) V^{P}-\partial^{M} \partial_{K} \xi^{L} \partial_{L} V^{K} .
\end{aligned}
$$

For the tensor we have $\delta_{\xi} T=\left[\int \Xi, T\right]$ which means that

$$
\frac{1}{2}\left(\delta_{\xi} T^{M N}\right) Z_{M} Z_{N}-\frac{1}{2}\left(\left(\delta_{\xi} \hat{T}^{M}\right) Z_{M}\right)^{\prime}=\left[\int \Xi, \frac{1}{2} T^{M N} Z_{M} Z_{N}-\frac{1}{2}\left(\hat{T}^{M} Z_{M}\right)^{\prime}\right] .
$$

The computation of the first contribution on the right-hand side gives

$$
\left[\int \Xi, T Z Z\right]=\left(\delta_{\xi} T^{M N}\right) Z_{M} Z_{N}-\left[\left(\Delta_{\xi} \hat{T}^{M}\right) Z_{M}\right]^{\prime},
$$

where the gauge transformation of the two index tensor gets determined to be

$$
\begin{aligned}
\delta_{\xi} T^{M N}= & \xi^{P} \partial_{P} T^{M N}+\left(\partial^{M} \xi_{P}-\partial_{P} \xi^{M}\right) T^{P N}+\left(\partial^{N} \xi_{P}-\partial_{P} \xi^{N}\right) T^{M P} \\
& -\frac{1}{2}\left[\partial^{N} T_{Q}^{P} \partial_{P} \partial^{[Q} \xi^{M]}+2 \partial_{Q} T^{K M} \partial^{N} \partial_{K} \xi^{Q}+(M \leftrightarrow N)\right] \\
& -\frac{1}{4} \partial_{K} \partial^{(M} T^{P Q} \partial^{N)} \partial_{P} \partial_{Q} \xi^{K}
\end{aligned}
$$

and the extra piece, showing the necessity of the pseudovector part, is found to be

$$
\Delta_{\xi} \hat{T}^{M}=-T_{P Q} \partial^{P} \partial^{[Q} \xi^{M]}-\frac{1}{2} \partial_{K} T^{P Q} \partial^{M} \partial_{P} \partial_{Q} \xi^{K} .
$$

The second contribution on the right-hand side of (4.58) gives

$$
\left[\int \Xi,(\hat{T} Z)^{\prime}\right]=\left[\int \Xi, \hat{T} Z\right]^{\prime}=\left([\Xi, \hat{T}]_{D}^{M} Z_{M}\right)^{\prime},
$$


where $[\Xi, \hat{T}]_{D}$ is the transformation the pseudovector $\hat{T}$ would have if it were a true vector. All in all we have

$$
\delta_{\xi} \hat{T}^{M}=[\Xi, \hat{T}]_{D}^{M}+\Delta_{\xi} \hat{T}^{M}
$$

and therefore

$$
\delta_{\xi} \hat{T}^{M}=[\Xi, \hat{T}]_{D}^{M}-T_{P Q} \partial^{P} \partial^{[Q} \xi^{M]}-\frac{1}{2} \partial_{K} T^{P Q} \partial^{M} \partial_{P} \partial_{Q} \xi^{K}
$$

This completes our determination of the gauge transformation of the tensor $T$. Note that : $V W$ : is a particular case of tensor $T$. Also, $\delta_{\xi} T^{M N}$ depends only on $T^{M N}$ while $\delta_{\xi} \hat{T}^{M}$ depends both on $\hat{T}^{M}$ and $T^{M N}$. This means that $T^{M N}$ and $\left(T^{M N}, \hat{T}^{M}\right)$ are both representations, but $\hat{T}^{M}$ by itself is not. $T$ is "not fully reducible", as e.g., the adjoint representation of the Poincaré group.

\section{Dilaton and double volume}

In this section we introduce and study the Virasoro tensor operator $\mathcal{S}$ that involves the dilaton field. Virasoro operators are tensor operators that generate conformal symmetries. This kind of symmetry transformations takes the form discussed earlier in (3.4) and the following paragraphs. Thus associated with a tensor operator $T$ we have the operator

$$
\Lambda(1)=\lambda(1) T(1)=\lambda\left(z_{1}\right) \frac{1}{2}\left[T^{M N}(X) Z_{M} Z_{N}-\left(\hat{T}^{M} Z_{M}\right)^{\prime}\right]\left(z_{1}\right),
$$

obtained by multiplying the tensor by a world sheet parameter $\lambda(z)$. The corresponding symmetry transformation $\delta_{\lambda} B$ of any operator $B$ is defined by the commutator

$$
\delta_{\lambda} B=\left[\int \Lambda, B\right] .
$$

The closure of this symmetry algebra, with one or more tensors involved, is quite nontrivial and requires conditions that can be interpreted as field equations for the components of the tensor operators. This will be the subject of the next section, where we introduce a second Virasoro operator $\mathcal{T}$ that encodes the gravitational variables of the theory.

\subsection{Virasoro operator $\mathcal{S}$}

As mentioned earlier, the worldsheet Hamiltonian is given by $\int \frac{1}{2} Z^{2}$. The two-dimensional energy-momentum tensor $\frac{1}{2} Z^{2}$ can have a total-derivative "improvement term". Such a term is implied by the coupling of the dilaton to the worldsheet curvature and is proportional to $\partial_{ \pm}^{2} \phi[2-4]$. We therefore take the tensor operator $\mathcal{S}$ to be given by

$$
\mathcal{S} \equiv \frac{1}{2}\left(Z^{2}-\phi^{\prime \prime}\right) \quad \Rightarrow \quad T^{M N}=\eta^{M N}, \hat{T}^{M}=\partial^{M} \phi,
$$

where we indicated the tensor components to the right.

The gauge transformations calculated in the previous section allow us to determine the dilaton gauge transformation. We see from (4.60) that our choice $T^{M N}=\eta^{M N}$ is 
consistent as the right-hand side of that equation vanishes for such $T^{M N}$. Equation (4.64) then gives us

$$
\begin{aligned}
\delta_{\xi}\left(\partial^{M} \phi\right)=\partial^{M}\left(\delta_{\xi} \phi\right) & =[\Xi, \partial \phi]_{D}^{M}+\eta_{P Q} \partial^{P} \partial^{M} \xi^{Q} \\
& =\xi^{K} \partial_{K} \partial^{M} \phi+\partial^{M} \xi^{K} \partial_{K} \phi+\partial^{M}(\partial \cdot \xi) \\
& =\partial^{M}\left(\xi^{K} \partial_{K} \phi+\partial \cdot \xi\right)
\end{aligned}
$$

where we used (4.43). We then conclude that

$$
\delta_{\xi} \phi=\xi \cdot \partial \phi+\partial \cdot \xi,
$$

unmodified from the classical result. This means that $e^{\phi}$ transforms as the "volume element" (measure) for spacetime integration. For example, as in conventional gravity, it can be used to define the divergence of a vector without using a metric. We'll see next that this also allows $T$ to be reduced by fixing $\hat{T}$ in terms of $T^{M N}$.

(We have dropped a constant that can be added to the right-hand side of (5.5), given that only the derivative of the gauge transformation is determined. The associated transformation $\delta \phi=\gamma$, with constant $\gamma$ and independent of $\Xi$, leaves field equations invariant but scales the action through the $e^{\phi}$ factor in the measure, showing that dilaton shifts change the coupling constant.)

Without the dilaton improvement term the following OPE holds for the operator $\frac{1}{2} Z^{2}$ :

$$
\frac{1}{2} Z^{2}(1) \frac{1}{2} Z^{2}(2)=\frac{D}{z_{12}^{4}}+\frac{Z^{2}(2)}{z_{12}^{2}}+\frac{\left(\frac{1}{2} Z^{2}\right)^{\prime}(2)}{z_{12}}+\text { finite. }
$$

Using this result, some additional calculation (with repeated use of the strong constraint) gives the remarkable fact that the OPE of the improved operators $\mathcal{S}$ is exactly the same:

$$
\mathcal{S}(1) \mathcal{S}(2)=\frac{D}{z_{12}^{4}}+\frac{2 \mathcal{S}(2)}{z_{12}^{2}}+\frac{\mathcal{S}^{\prime}(2)}{z_{12}}+\text { finite }
$$

Next we consider products $\mathcal{S}(1) \mathcal{O}(2)$, expanded about $z_{2}$, for arbitrary operators $\mathcal{O}$. We first note that the least singular terms, $1 / z_{12}$ and $1 / z_{12}^{2}$, are completely classical: they are determined from terms with a single propagator contracting with $\frac{1}{2} Z^{2}$. If we used two propagators contracting with $\frac{1}{2} Z^{2}$ this leaves no $z_{1}$ dependence except in the $z_{12}$ 's, so nothing to expand about $z_{2}$. But then the only term less singular than $1 / z_{12}^{3}$ is killed by the strong constraint. For the $\phi^{\prime \prime}$ term, we contract $\phi$, Taylor expand about $z_{2}$, and take the $\partial_{1}^{2}$ from $\partial_{1}^{2} \phi(1)$ to act last. This gives terms of the form

$$
\begin{aligned}
& \partial_{1}^{2}\left[\frac{1}{z_{12}^{n}}\left(\partial_{M} \ldots \partial_{N} \phi\right)(1) \mathcal{O}^{M \ldots N}(2)\right] \\
& \quad=\partial_{1}^{2}\left[\frac{1}{z_{12}^{n}}\left(\partial_{M} \ldots \partial_{N} \phi\right) \mathcal{O}^{M \ldots N}(2)+\frac{1}{z_{12}^{n-1}}\left(\partial_{M} \ldots \partial_{N} \phi\right)^{\prime}(2) \mathcal{O}^{M \ldots N}(2)+\ldots\right] .
\end{aligned}
$$

But $\partial_{1}^{2}$ on any negative power of $z$ will yield terms at least as singular as $1 / z^{3}$. This is true for any number of propagators: $\phi$ has no classical contribution to the $1 / z_{12}$ and $1 / z_{12}^{2}$ terms either. We then have

$$
\frac{1}{2} Z^{2}(1) \mathcal{O}(2)=\ldots+\frac{w_{\mathcal{O}} \mathcal{O}(2)}{\left(z_{12}\right)^{2}}+\frac{\mathcal{O}^{\prime}(2)}{z_{12}}+\text { finite },
$$


which imply

$$
\mathcal{S} \circ_{w_{\mathcal{O}}+1} \mathcal{O}=\mathcal{O}^{\prime}, \quad \mathcal{S} \circ_{w_{\mathcal{O}}} \mathcal{O}=w_{\mathcal{O}} \mathcal{O} .
$$

For the two next least divergent terms we make the definitions of the quantum generalizations of the trace and divergence:

$$
\operatorname{div}(\mathcal{O}) \equiv \mathcal{S} \circ_{w_{\mathcal{O}}-1} \mathcal{O}, \quad \frac{1}{2} \operatorname{tr}(\mathcal{O}) \equiv \mathcal{S} \circ_{w_{\mathcal{O}}-2} \mathcal{O}
$$

The divergence lowers the weight by one, the trace lowers the weight by two. We can apply the derivative identities to the above general expressions for $\mathcal{S} \circ$. For the latter cases we find

$$
\begin{aligned}
\operatorname{tr}\left(\mathcal{O}^{\prime}\right) & =(\operatorname{tr} \mathcal{O})^{\prime}+6 \operatorname{div} \mathcal{O}, \\
\operatorname{div}\left(\mathcal{O}^{\prime}\right) & =(\operatorname{div} \mathcal{O})^{\prime}+2 w_{\mathcal{O}} \mathcal{O},
\end{aligned}
$$

while the $\circ_{w_{\mathcal{O}}+1}$ identity is trivial and the $\circ_{w_{\mathcal{O}}}$ identity shows that the expression for $\circ_{w_{\mathcal{O}}+1}$ is implied by that for $\circ_{w_{\mathcal{O}}}$.

For the tensor $T$ the trace gives a scalar. For the vector $V$ the divergence gives a scalar and the trace gives zero. For the scalar $f$ both the trace and the divergence give zero. Thus

$$
\operatorname{tr} V=\operatorname{tr} f=\operatorname{div} f=0 .
$$

The derivative identities then specialize:

$$
\begin{aligned}
\operatorname{tr}\left(V^{\prime}\right) & =6 \operatorname{div} V \\
\operatorname{div}\left(V^{\prime}\right) & =2 V+(\operatorname{div} V)^{\prime} \\
\operatorname{div}\left(f^{\prime}\right) & =0 .
\end{aligned}
$$

We can write these products collectively as the OPE

$$
\mathcal{S}(1) \mathcal{O}(2)=\text { finite }+\frac{1}{z_{12}} \mathcal{O}^{\prime}+\frac{1}{z_{12}^{2}} w_{\mathcal{O}} \mathcal{O}+\frac{1}{z_{12}^{3}} \operatorname{div}(\mathcal{O})+\frac{1}{z_{12}^{4}} \frac{1}{2} \operatorname{tr}(\mathcal{O})+\ldots,
$$

so that conformal transformations take the form

$$
\left[\int \lambda \mathcal{S}, \mathcal{O}\right]=\lambda \mathcal{O}^{\prime}+w_{\mathcal{O}} \lambda^{\prime} \mathcal{O}+\frac{1}{2} \lambda^{\prime \prime} \operatorname{div}(\mathcal{O})+\frac{1}{12} \lambda^{\prime \prime \prime} \operatorname{tr}(\mathcal{O})+\ldots,
$$

where the first two terms are the usual (free, "on-shell") universal terms.

Straightforward calculation gives the covariants

$$
\begin{aligned}
\operatorname{tr} T & =\eta^{M N} T_{M N}-3\left(T^{M N} \partial_{M} \partial_{N} \phi+\partial \cdot \hat{T}+\hat{T} \cdot \partial \phi\right), \\
(\operatorname{div} T)^{M} & =\partial_{N} T^{M N}+T^{M N} \partial_{N} \phi-\frac{1}{2} T^{N P} \partial_{N} \partial_{P} \partial^{M} \phi-\hat{T}^{M}-\frac{1}{2} \partial^{M}(\partial \cdot \hat{T}+\hat{T} \cdot \partial \phi), \\
\operatorname{div} V & =\partial \cdot V+V \cdot \partial \phi,
\end{aligned}
$$

as well as the trivial cases

$$
\operatorname{tr}(\mathcal{S})=2 D, \quad \operatorname{div}(\mathcal{S})=0
$$


This explicit expression for the "divergence" of a vector also identifies $e^{\phi}$ as the integration measure, taking the place of " $\sqrt{-g}$ ":

$$
\operatorname{div} V=e^{-\phi} \partial \cdot\left(e^{\phi} V\right) .
$$

The case of a trivial tensor is of some interest. Such tensor is the $z$ derivative of a vector operator $V$ :

$$
T=V^{\prime}=\left(Z_{M} V^{M}\right)^{\prime} \Rightarrow \hat{T}^{M}=-2 V^{M}, \quad T_{M N}=0 .
$$

\subsection{Projection to divergence free tensors}

Before introduction of the dilaton, we found that operators of $w>1$ were not fully reducible. We'll see now a further reduction, the separation of the "divergence" and "divergenceless" pieces. In the case of weight-two tensors $T$, this allows us to treat $T^{M N}$ and $\hat{T}^{M}$ separately.

We therefore look for a solution to the constraint $\operatorname{div} \overline{\mathcal{O}}=0$ by projecting out the div piece of $\mathcal{O}$. The solution is not unique; we look for a solution by taking $z$-derivatives of iterated divergences

$$
\overline{\mathcal{O}}=\left(\sum_{n=0}^{w_{\mathcal{O}}} c_{n} \mathcal{A}^{n}\right) \mathcal{O}, \quad \mathcal{A}^{n} \mathcal{O} \equiv\left(\operatorname{div}^{n} \mathcal{O}\right)^{(n)}
$$

Here, for example $\mathcal{A}^{2} \mathcal{O}=(\operatorname{div} \operatorname{div} \mathcal{O})^{\prime \prime}$ and $\mathcal{A}^{0} \mathcal{O}=\mathcal{O}$. Note that the sum can be taken to $\infty$ since div vanishes on a scalar. Using the $\operatorname{div}\left(\mathcal{O}^{\prime}\right)$ identity, we find by induction

$$
\operatorname{div} \mathcal{A}^{n}=\mathcal{A}^{n} \operatorname{div}+2\left(n w_{\mathcal{O}}-\frac{n(n+1)}{2}\right) \mathcal{A}^{n-1} \operatorname{div} .
$$

This allows the constraint to be solved as (using recursion or differential equation)

$$
\overline{\mathcal{O}}=\overline{\overline{\mathcal{O}}}=g(\mathcal{A}) \mathcal{O}, \quad g(x)=\sum_{n=0}^{w_{\mathcal{O}}} \frac{\left[2\left(w_{\mathcal{O}}-1\right)-n\right] !}{n !\left[2\left(w_{\mathcal{O}}-1\right)\right] !}(-x)^{n}
$$

These polynomials are essentially the Neumann polynomials, or the leading terms in the modified Bessel functions of the second kind:

$$
\begin{aligned}
\frac{4}{(a+1) !} x^{a / 2+1} O_{a+1}(2 \sqrt{x}) & =\sum_{n=0}^{[(a+1) / 2]} \frac{(a-n) !}{n ! a !} x^{n} \\
\frac{2}{a !} x^{(a+1) / 2} K_{a+1}(2 \sqrt{x}) & =\sum_{n=0}^{a} \frac{(a-n) !}{n ! a !} x^{n}+\ldots
\end{aligned}
$$

Similarly,

$$
\mathcal{A}^{n} \partial_{z}=\partial_{z} \mathcal{A}^{n}+2\left(n w_{\mathcal{O}^{\prime}}-\frac{n(n-1)}{2}\right) \partial_{z} \mathcal{A}^{n-1}
$$

implies

$$
\overline{\mathcal{O}^{\prime}}=0 \text {. }
$$


The result is that for arbitrary-weight operators (except vectors, $w_{\mathcal{O}}=1$ ) we can initially ignore all total $z$-derivative terms, as they will be fixed in terms of the rest by the - operation.

In particular, this applies to all products $\mathcal{O}_{1} \circ_{w} \mathcal{O}_{2}$ for $w>1$. This means we can replace all "•" products defined previously by a new product with nicer properties: from the symmetry condition on $\circ$, we see that - automatically (anti)symmetrizes it,

$$
\mathcal{O}_{1} \bullet{ }_{w} \mathcal{O}_{2} \equiv \overline{\mathcal{O}_{1} \circ_{w} \mathcal{O}_{2}}=(-1)^{w_{1}+w_{2}-w} \mathcal{O}_{2} \bullet_{w} \mathcal{O}_{1}
$$

We then define the Div operation through the following relation

$$
\overline{\mathcal{O}} \equiv \mathcal{O}-\frac{1}{2\left(w_{\mathcal{O}}-1\right)}(\operatorname{Div}(\mathcal{O}))^{\prime},
$$

which implies that

$$
\operatorname{Div}\left(\mathcal{O}^{\prime}\right)=2 w_{\mathcal{O}} \mathcal{O}, \quad \operatorname{Div}(\overline{\mathcal{O}})=0,
$$

as well as

$$
\operatorname{Div} \mathcal{O}=h(\mathcal{A}) \operatorname{div} \mathcal{O}, \quad h(x)=\sum_{n=0}^{2 w_{\mathcal{O}}-3} \frac{\left[\left(2 w_{\mathcal{O}}-3\right)-n\right] !}{(n+1) !\left(2 w_{\mathcal{O}}-3\right) !}(-x)^{n}
$$

Note that $h(\mathcal{A})$ is an invertible finite polynomial. This means that div determines Div, and vice versa. In particular,

$$
\operatorname{div} \mathcal{O}=0 \quad \Leftrightarrow \quad \operatorname{Div} \mathcal{O}=0
$$

so the two constraints are freely interchangeable. The advantage of Div over div is that on $\mathcal{O}^{\prime}$, div gives $2 w_{\mathcal{O}} \mathcal{O}+(\operatorname{div} \mathcal{O})^{\prime}$, while Div gives simply the first term. This allows $\operatorname{Div} \mathcal{O}=0$ to be more easily solved than $\operatorname{div} \mathcal{O}=0$, although the solution is the same.

In particular,

$$
\bar{T}=T-\frac{1}{2}(\operatorname{Div} T)^{\prime}, \quad \operatorname{Div} T=\operatorname{div} T-\frac{1}{2}\left(\operatorname{div}^{2} T\right)^{\prime},
$$

so that

$$
\bar{T}=T-\frac{1}{2}(\operatorname{div} T)^{\prime}+\frac{1}{4}\left(\operatorname{div}^{2} T\right)^{\prime \prime} .
$$

Thus, since the terms subtracted affect only the pseudovector part

$$
\bar{T}^{M N}=T^{M N} .
$$

Using the explicit expressions for the divergence of a tensor and of a vector we find

$$
\begin{aligned}
(\operatorname{Div} T)^{M}= & -\hat{T}^{M}+\left(\partial_{N} T^{M N}+T^{M N} \partial_{N} \phi\right)-\frac{1}{2} T^{N P} \partial_{N} \partial_{P} \partial^{M} \phi \\
& -\frac{1}{2} \partial^{M}\left\{\partial_{N} \partial_{P} T^{N P}+T^{N P}\left[\partial_{N} \partial_{P} \phi+\left(\partial_{N} \phi\right)\left(\partial_{P} \phi\right)\right]\right\}
\end{aligned}
$$


The expression for $\widehat{\bar{T}}$ is equal to the value of $\hat{T}$ for which $\operatorname{Div} T=0$. From the above equation we get

$$
\operatorname{Div} T=0 \Rightarrow \hat{T}^{M}=G^{M}\left(T^{M N}, \phi\right),
$$

where we have introduced the vector function

$$
\begin{aligned}
G^{M}\left(T^{M N}, \phi\right) \equiv\left(\partial_{N} T^{M N}\right. & \left.+T^{M N} \partial_{N} \phi\right)-\frac{1}{2} T^{N P} \partial_{N} \partial_{P} \partial^{M} \phi \\
& -\frac{1}{2} \partial^{M}\left\{\partial_{N} \partial_{P} T^{N P}+T^{N P}\left[\partial_{N} \partial_{P} \phi+\left(\partial_{N} \phi\right)\left(\partial_{P} \phi\right)\right]\right\} .
\end{aligned}
$$

The tensor $\bar{T}$ is thus given by

$$
\bar{T}=\frac{1}{2} T^{M N} Z_{M} Z_{N}-\frac{1}{2}\left[G^{M}\left(T^{M N}, \phi\right) Z_{M}\right]^{\prime} .
$$

Note that the pseudovector part $\hat{T}^{M}$ of $T$ has dropped out of $\bar{T}$, while appearing in $\operatorname{Div} T$ in the simplest nontrivial way. The divergenceless tensor $\bar{T}$ has a pseudovector part but it is determined in terms of $T^{M N}$ and the dilaton through the function $G$.

Another useful evaluated expression is

$$
\begin{aligned}
\operatorname{tr}(\bar{T}) & =\operatorname{tr} T-3 \operatorname{div}^{2} T \\
& =\eta^{M N} T_{M N}-3\left[T^{M N} \partial_{M} \phi \partial_{N} \phi+\partial_{M}\left(\partial_{N} T^{M N}+2 T^{M N} \partial_{N} \phi\right)\right] .
\end{aligned}
$$

We also have the trivial case

$$
\operatorname{Div} \mathcal{S}=0 \quad \Rightarrow \quad \overline{\mathcal{S}}=\mathcal{S}
$$

\section{Double metric}

Having studied the properties of the tensor operator $\mathcal{S}$ encoding the dilaton background, we now introduce the second Virasoro (tensor) operator $\mathcal{T}$ that encodes the gravitational background. We take, in full generality

$$
T^{M N}=\mathcal{M}^{M N}, \quad \hat{T}^{M}=\widehat{\mathcal{M}}^{M} \Rightarrow \mathcal{T}=\frac{1}{2}\left[\mathcal{M}^{M N} Z_{M} Z_{N}-\left(\widehat{\mathcal{M}}^{M} Z_{M}\right)^{\prime}\right] .
$$

In here the field $\mathcal{M}^{M N}$ will be called the double metric. Nothing is assumed about it to begin. The field $\widehat{\mathcal{M}}^{M}$ is an additional degree of freedom that will eventually get determined in terms of the double metric and the dilaton.

\subsection{Field equations}

The field equations for $\mathcal{M}^{M N}, \widehat{\mathcal{M}}^{M}$, and the dilaton appear as enforcement of the Virasoro algebra for the operators $\mathcal{S}$ and $\mathcal{T}$. Since only singular terms contribute to commutators, we look at the table of products $T \circ_{w} T$ only for $w \leq 3$. The Virasoro algebra requires:

$$
\begin{aligned}
& w=\begin{array}{llll}
0 & 1 & 2 & 3
\end{array}
\end{aligned}
$$

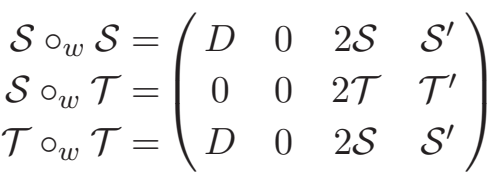


(Note that $\mathrm{O}_{2}$ is a quantum generalization of the anticommutator when applied to tensors $T$ : e.g., $\mathcal{S} \circ_{2} T=2 T$.) Ghost contributions, which we don't discuss in this paper, would cancel the $\langle\mathcal{S} \mid \mathcal{S}\rangle$ and $\langle\mathcal{T} \mid \mathcal{T}\rangle$ terms. The ghosts are not necessary for the classical field theory we are building, presumably because they do not couple to the background. The $\mathcal{S S}$ equations are satisfied off shell, as discussed earlier. The $\mathcal{S T}$ equations for $\circ_{3}$ and $\circ_{2}$ also hold off-shell (see (5.10)). The ones for $\circ_{1}$ and $\circ_{0}$ are, respectively,

$$
\begin{array}{rlrl}
\operatorname{div}(\mathcal{T}) & =0: & \widehat{\mathcal{M}}^{M} \\
\operatorname{tr}(\mathcal{T}) & =0: \quad \phi .
\end{array}
$$

The first equation fixes $\widehat{\mathcal{M}}^{M}=G^{M}\left(\mathcal{M}^{M N}, \phi\right)$, as defined in (5.36). The second equation can be viewed as the dilaton field equation. Let us now consider the $\mathcal{T} \mathcal{T}$ equations. By the symmetry identity, $\mathcal{T} \circ_{3} \mathcal{T}$ and $\mathcal{T} \circ_{1} \mathcal{T}$ are derivatives of $\mathcal{T} \circ_{2} \mathcal{T}$ and $\mathcal{T} \circ_{0} \mathcal{T}$, so only the latter are relevant. The $\circ_{2}$ condition $\mathcal{T} \circ_{2} \mathcal{T}=2 \mathcal{S}$ gives two equations and the $\circ_{0}$ condition just one:

$$
\begin{array}{rlrl}
\left(\mathcal{T} \circ_{2} \mathcal{T}\right)^{M N} & =2 \eta^{M N} & : & \mathcal{M}^{M N} \\
\left(\mathcal{T} \circ_{2} \mathcal{T}\right)^{M}=2 \partial^{M} \phi & : & \text { redundant } \\
\langle\mathcal{T} \mid \mathcal{T}\rangle & =D & : & \text { redundant }
\end{array}
$$

The first is a nontrivial equation for the field $\mathcal{M}^{M N}$; the last two are redundant to the first and those in (6.3), as we now show.

We first reorganize a bit the equations above. Since $\operatorname{div} \mathcal{T}=0$ we have $\overline{\mathcal{T}}=\mathcal{T}$. We can then let $\mathcal{T} \rightarrow \overline{\mathcal{T}}$ everywhere thus taking care of the first equation in (6.3). Note also that the vanishing of any tensor $T$ is equivalent to the vanishing of $\bar{T}$ and the vanishing of $\operatorname{div} T$ (or alternatively, the vanishing of $\bar{T}$ and $\operatorname{Div} T$ ). We do this with the $\mathcal{T} \circ_{2} \mathcal{T}=2 \mathcal{S}$ equation, recalling that $\overline{\mathcal{S}}=\mathcal{S}$. We then have

$$
\begin{array}{rll}
\operatorname{tr}(\overline{\mathcal{T}})=0 & : & \phi, \\
\overline{\overline{\mathcal{T}} \circ_{2} \overline{\mathcal{T}}}=2 \mathcal{S} & : & \mathcal{M}^{M N}, \\
\operatorname{div}\left(\overline{\mathcal{T}} \circ_{2} \overline{\mathcal{T}}\right)=0 & : & \text { redundant } \\
\langle\overline{\mathcal{T}} \mid \overline{\mathcal{T}}\rangle=D & : & \text { redundant }
\end{array}
$$

Consider again the distributivity identities, now for $\mathcal{O}_{1}=\mathcal{S}, \mathcal{O}_{2}=T_{1}, \mathcal{O}_{3}=T_{2}$, so $w_{i}=2$, and also $w=2$, but $\hat{w}=0$ or 1 . Then

$$
\mathcal{S} \circ_{\hat{w}}\left(T_{1} \circ_{2} T_{2}\right)-T_{1} \circ_{\hat{w}}\left(\mathcal{S} \circ_{\hat{w}} T_{2}\right)=\sum_{w^{\prime \prime}=1}^{4-\hat{w}}\left(\begin{array}{c}
3-\hat{w} \\
w^{\prime \prime}-1
\end{array}\right)\left(\mathcal{S} \circ_{4-w^{\prime \prime}} T_{1}\right) \circ_{\hat{w}} T_{2} .
$$

Using the $\mathcal{S} \circ$ and derivative identities,

$$
\begin{aligned}
\operatorname{tr}\left(T_{1} \circ_{2} T_{2}\right) & =T_{1} \circ_{0}\left(\operatorname{tr} T_{2}\right)+\left(\operatorname{tr} T_{1}\right) \circ_{0} T_{2}+6\left(\operatorname{div} T_{1}\right) \circ_{0} T_{2}+4\left\langle T_{1} \mid T_{2}\right\rangle, \\
\operatorname{div}\left(T_{1} \circ_{2} T_{2}\right) & =T_{1} \circ_{1}\left(\operatorname{div} T_{2}\right)+\left(\operatorname{div} T_{1}\right) \circ_{1} T_{2}+T_{1} \circ_{1} T_{2} .
\end{aligned}
$$


Noting that $T \circ_{1} T=\frac{1}{2}\langle T \mid T\rangle^{\prime}$ (symmetry identity) and setting $T_{1}=T_{2}=\bar{T}$, we get

$$
\begin{aligned}
\operatorname{tr}\left(\bar{T} \circ_{2} \bar{T}\right) & =2\langle\operatorname{tr} \bar{T} \mid \bar{T}\rangle+4\langle\bar{T} \mid \bar{T}\rangle, \\
\operatorname{div}\left(\bar{T} \circ_{2} \bar{T}\right) & =\frac{1}{2}\langle\bar{T} \mid \bar{T}\rangle^{\prime} .
\end{aligned}
$$

The first can be re-expressed using (5.39) and the second, to find

$$
\operatorname{tr}\left(\overline{\bar{T} \circ_{2} \bar{T}}\right)=2\langle\operatorname{tr} \bar{T} \mid \bar{T}\rangle+4\langle\bar{T} \mid \bar{T}\rangle .
$$

Substituting $\mathcal{T}$ for $T$ and applying the $\mathcal{M}$ and $\phi$ field equations, the last two equations become

$$
\begin{aligned}
\operatorname{div}\left(\overline{\mathcal{T}} \circ_{2} \overline{\mathcal{T}}\right) & =\frac{1}{2}\langle\overline{\mathcal{T}} \mid \overline{\mathcal{T}}\rangle^{\prime}, \\
4 D & =4\langle\overline{\mathcal{T}} \mid \overline{\mathcal{T}}\rangle,
\end{aligned}
$$

proving, as we wanted, that the last two equations in (6.5) are redundant.

\section{$6.2 \star$ product}

In this subsection we consider a number of properties that will allow us to write an action and vary it to determine its field equations. A useful star-product will be introduced. This product yields weight-two divergence-free tensors. It is also symmetric, and together with the inner product defines a scalar that is totally symmetric in its three tensor inputs.

The action will take the form $\int e^{\phi} L$ where $L$ is a scalar. As noted earlier, for an arbitrary vector $V$ integration by parts shows that

$$
\int e^{\phi} \operatorname{div} V=0
$$

It is convenient to introduce the equivalence symbol $\sim$ for objects that are the same under the integral

$$
A \sim B \quad \rightarrow \quad \int e^{\phi} A=\int e^{\phi} B
$$

We thus have

$$
\operatorname{div} V \sim 0
$$

Since $\operatorname{tr}\left(V^{\prime}\right)=6 \operatorname{div} V$ we also have

$$
\operatorname{tr}\left(V^{\prime}\right) \sim 0
$$

which states that the trace of a trivial tensor gives no contribution to the action. Since $\bar{T}=T+V^{\prime}$ for some $V$, we also have that

$$
\operatorname{tr}(T) \sim \operatorname{tr}(\bar{T}) .
$$

We now use the distributive identity (3.33) with $\mathcal{O}_{1}=\mathcal{S}, \mathcal{O}_{2}=\mathcal{O}, \mathcal{O}_{3}=T$, and $\hat{w}=0, w^{\prime}=3$ :

$$
\operatorname{div}\left(\mathcal{O} \circ_{1} T\right)=\langle\operatorname{div} \mathcal{O} \mid T\rangle+\langle\mathcal{O} \mid \operatorname{div} T\rangle+\left(w_{\mathcal{O}}-2\right)\langle\mathcal{O} \mid T\rangle
$$


where we also used the identifications (A.3). For the cases of the scalar, vector, and tensor, we get

$$
\begin{aligned}
\operatorname{div}\left(f \circ_{1} T\right) & =\langle f \mid \operatorname{div} T\rangle-2\langle f \mid T\rangle, \\
\operatorname{div}\left(V \circ_{1} T\right) & =\langle\operatorname{div} V \mid T\rangle+\langle V \mid \operatorname{div} T\rangle-\langle V \mid T\rangle, \\
\operatorname{div}\left(T_{1} \circ_{1} T_{2}\right) & =\left\langle\operatorname{div} T_{1} \mid T_{2}\right\rangle+\left\langle T_{1} \mid \operatorname{div} T_{2}\right\rangle .
\end{aligned}
$$

Applied to divergenceless tensors $\bar{T}$ we have

$$
\begin{aligned}
\operatorname{div}\left(f \circ_{1} \bar{T}\right) & =-2\langle f \mid \bar{T}\rangle, \\
\operatorname{div}\left(V \circ_{1} \bar{T}\right) & =\langle\operatorname{div} V \mid \bar{T}\rangle-\langle V \mid \bar{T}\rangle, \\
\operatorname{div}\left(\bar{T}_{1} \circ_{1} \bar{T}_{2}\right) & =0 .
\end{aligned}
$$

The first equation implies that

$$
\langle\bar{T} \mid f\rangle \sim 0 .
$$

The second equation, using the first, can be written as

$$
\langle V \mid \bar{T}\rangle=-\operatorname{div}\left[V \circ_{1} \bar{T}+\frac{1}{2}(\operatorname{div} V) \circ_{1} \bar{T}\right],
$$

which implies that $\langle\bar{T} \mid V\rangle \sim 0$. Thus, all in all,

$$
\langle\bar{T} \mid f\rangle \sim 0, \quad\langle\bar{T} \mid V\rangle \sim 0 .
$$

Thus divergenceless tensors have the remarkable property that their inner product against a scalar or a vector are zero under the integral. We now note that the overlap of a projected tensor $\bar{T}_{1}$ and an unprojected tensor $T_{2}$ picks up its projected part:

$$
\left\langle\bar{T}_{1} \mid T_{2}\right\rangle=\left\langle\bar{T}_{1} \mid \bar{T}_{2}+V^{\prime}\right\rangle \sim\left\langle\bar{T}_{1} \mid \bar{T}_{2}\right\rangle,
$$

where we used $\left\langle\bar{T} \mid V^{\prime}\right\rangle=\left\langle V^{\prime} \mid \bar{T}\right\rangle=-3\langle V \mid \bar{T}\rangle \sim 0$. The overline projection is an orthogonal projection.

We now show that there are two equivalent ways of forming a scalar in order to use it in the action. From (6.7) we have

$$
\operatorname{tr}\left(\bar{T}_{1} \circ_{2} \bar{T}_{2}\right)=\left\langle\bar{T}_{(1} \mid \operatorname{tr} \bar{T}_{2)}\right\rangle+4\left\langle\bar{T}_{1} \mid \bar{T}_{2}\right\rangle .
$$

The first term on the right-hand side is equivalent to zero under the integral on account of (6.19) so that

$$
\left\langle\bar{T}_{1} \mid \bar{T}_{2}\right\rangle \sim \frac{1}{4} \operatorname{tr}\left(\bar{T}_{1} \circ_{2} \bar{T}_{2}\right) .
$$

Recall now our definition of symmetric products $\bullet_{w}$ in (5.27). The case $w=2$, for which the output (regardless of the inputs) is a tensor, will be particularly useful. We will call this product a "star" product: $\star \equiv \bullet_{2}$. We thus have:

$$
\mathcal{O}_{1} \star \mathcal{O}_{2} \equiv \overline{\mathcal{O}_{1} \circ_{2} \mathcal{O}_{2}} \text {. }
$$


Using this notation and recalling (6.15), we see that (6.24) takes the form

$$
\left\langle\bar{T}_{1} \mid \bar{T}_{2}\right\rangle \sim \frac{1}{4} \operatorname{tr}\left(\bar{T}_{1} \star \bar{T}_{2}\right) .
$$

To perform the variation of the action we need to show that under the integral $\left\langle T_{1}\right| T_{2} \star$ $\left.T_{3}\right\rangle$ is totally symmetric when $\operatorname{div}\left(T_{i}\right)=0$. So we look at distributivity identities for three tensors $\left(w_{i}=2\right)$ with an inner product outside $(\hat{w}=0)$ and a $\circ_{2}$ inside. All these identities have a term with $\circ_{3}$ also; the ones with only one such term, appearing with the same coefficient, (and no $\left.\circ_{4}\right)$ are those with $\left(w, w^{\prime}\right)=(4,2),(3,3),(2,4)$. The former two are the simplest; taking their difference, we find

$$
\left\langle T_{2} \mid T_{1} \circ_{2} T_{3}\right\rangle-\left\langle T_{3} \mid T_{1} \circ_{2} T_{2}\right\rangle=\left\langle T_{2} \mid T_{1} \circ_{1} T_{3}\right\rangle-\left\langle T_{3} \mid T_{1} \circ_{1} T_{2}\right\rangle-\left\langle T_{1} \mid T_{2} \circ_{1} T_{3}\right\rangle+\left\langle T_{1} \mid\left\langle T_{2} \mid T_{3}\right\rangle\right\rangle .
$$

Applying this identity to divergenceless tensors, all terms on the right-hand side are equivalent to zero on account of (6.21) and therefore

$$
\left\langle\bar{T}_{1} \mid \bar{T}_{2} \circ_{2} \bar{T}_{3}\right\rangle \sim\left\langle\bar{T}_{3} \mid \bar{T}_{2} \circ_{2} \bar{T}_{1}\right\rangle .
$$

Now note that

$$
T_{1} \star T_{2}=\overline{T_{1} \circ_{2} T_{2}}=T_{1} \circ_{2} T_{2}+V^{\prime},
$$

for some vector $V$. Since $\left\langle\bar{T} \mid V^{\prime}\right\rangle \sim 0$, replacing $\circ_{2}$ with $\star$ has no effect on the above symmetry:

$$
\left\langle\bar{T}_{1} \mid \bar{T}_{2} \star \bar{T}_{3}\right\rangle \sim\left\langle\bar{T}_{3} \mid \bar{T}_{2} \star \bar{T}_{1}\right\rangle
$$

Since the product $\star$ is symmetric, this shows that $\left\langle\bar{T}_{1} \mid \bar{T}_{2} \star \bar{T}_{3}\right\rangle$ is totally symmetric. Note that the form in (6.28) is also totally symmetric because the product $\mathrm{O}_{2}$ is symmetric up to $z$-derivatives.

As a useful exercise we consider the explicit form of the star product of two projected tensors:

$$
\bar{T}_{1} \star \bar{T}_{2}=\bar{T}_{1} \circ_{2} \bar{T}_{2}-\frac{1}{2}\left(\operatorname{div}\left(\bar{T}_{1} \circ_{2} \bar{T}_{2}\right)\right)^{\prime}+\frac{1}{4}\left(\operatorname{div} \operatorname{div}\left(\bar{T}_{1} \circ_{2} \bar{T}_{2}\right)\right)^{\prime \prime} .
$$

We then note that $\operatorname{div}\left(\bar{T}_{1} \circ_{2} \bar{T}_{2}\right)=\bar{T}_{1} \circ_{1} \bar{T}_{2}$, because of (6.7), and that the last term above drops out by the last of (6.18). As a result, we have the simplified form

$$
\bar{T}_{1} \star \bar{T}_{2}=\bar{T}_{1} \circ_{2} \bar{T}_{2}-\frac{1}{2}\left(\bar{T}_{1} \circ_{1} \bar{T}_{2}\right)^{\prime} .
$$

When the two tensors are the same, further simplification is possible using the symmetry property,

$$
\bar{T} \star \bar{T}=\bar{T} \circ_{2} \bar{T}-\frac{1}{4}\langle\bar{T} \mid \bar{T}\rangle^{\prime \prime}
$$




\subsection{Action}

We use the double metric defined previously,

$$
\mathcal{T}=\frac{1}{2}\left[\mathcal{M}^{M N} Z_{M} Z_{N}-\left(\widehat{\mathcal{M}}^{M} Z_{M}\right)^{\prime}\right]
$$

with the condition that

$$
\operatorname{div} \mathcal{T}=0 \Rightarrow \overline{\mathcal{T}}=\mathcal{T}
$$

so that the identities for barred tensors can be used for $\mathcal{T}$. The tensor field $\mathcal{T}$ so constrained is only a function of $\mathcal{M}^{M N}$ and the dilaton. This means that $\widehat{\mathcal{M}}^{M}=G^{M}\left(\mathcal{M}^{M N}, \phi\right)$ is determined in terms of $\mathcal{M}^{M N}$ and the dilaton.

We now claim that the action is given by

$$
S=\int e^{\phi} L, \quad L=\left\langle\mathcal{T} \mid \mathcal{S}-\frac{1}{6} \mathcal{T} \star \mathcal{T}\right\rangle .
$$

Using (6.26) we also have the alternative form, equivalent up to total derivatives

$$
L=\frac{1}{2} \operatorname{tr}\left[\mathcal{T}-\frac{1}{12} \mathcal{T} \star(\mathcal{T} \star \mathcal{T})\right]
$$

This action is gauge invariant because the dilaton provides a measure and $L$ is a gauge scalar. This is clear by construction since we begin with tensors under gauge transformations and all our operations are covariant: the products, projections, inner products. The gauge transformations are simply

$$
\begin{aligned}
& \delta_{\xi} \mathcal{T}=\Xi \circ_{2} \mathcal{T}, \\
& \delta_{\xi} \mathcal{S}=\Xi \circ_{2} \mathcal{S} .
\end{aligned}
$$

The gauge transformed $\mathcal{T}$ is divergence free with respect to the divergence operator that uses the gauge transformed dilaton. The explicit form of the gauge transformations can be read from (4.60) and (5.5), and for completeness we give them here:

$$
\begin{aligned}
\delta_{\xi} \mathcal{M}^{M N}= & \xi^{P} \partial_{P} \mathcal{M}^{M N}+\left(\partial^{M} \xi_{P}-\partial_{P} \xi^{M}\right) \mathcal{M}^{P N}+\left(\partial^{N} \xi_{P}-\partial_{P} \xi^{N}\right) \mathcal{M}^{M P} \\
& -\frac{1}{2}\left[\partial^{M} \mathcal{M}^{P Q} \partial_{P}\left(\partial_{Q} \xi^{N}-\partial^{N} \xi_{Q}\right)+2 \partial_{Q} \mathcal{M}^{K M} \partial^{N} \partial_{K} \xi^{Q}+(M \leftrightarrow N)\right] \\
& -\frac{1}{4} \partial_{K} \partial^{\left(M^{M} \mathcal{M}^{P Q} \partial^{N)} \partial_{P} \partial_{Q} \xi^{K}\right.} \\
\delta_{\xi} \phi= & \xi \cdot \partial \phi+\partial \cdot \xi
\end{aligned}
$$

We now vary the action to derive the equations of motion. Consider first the variation $\delta_{\mathcal{M}}$ of double metric $\mathcal{M}_{M N}$. The only field to vary is $\mathcal{T}$ and $\delta_{\mathcal{M}} \mathcal{T}$ is still projected. The result is

$$
\delta_{\mathcal{M}} S=\int e^{\phi}\left\langle\delta_{\mathcal{M}} \mathcal{T} \mid \mathcal{S}-\frac{1}{2} \mathcal{T} \star \mathcal{T}\right\rangle
$$

using the total symmetry of $\int e^{\phi}\left\langle\bar{T}_{1} \mid \bar{T}_{2} \star \bar{T}_{3}\right\rangle$. Now note that $\left\langle\delta T_{1} \mid T_{2}\right\rangle$ gives $A T_{2}=0$ for some operator $A$ of the form

$$
A=I+\alpha^{\prime} A_{1}+\alpha^{\prime 2} A_{2}
$$


where $A_{1}$ is second-order in spacetime derivatives and $A_{2}$ is fourth-order. There are no higher-order terms because of the strong constraint: derivatives can be contracted only with the indices on $T$. (This includes derivatives acting on $\phi$.) For the same reason, $A$ is easily invertible, also terminating at fourth-order. Thus $A T_{2}=0$ implies $T_{2}=0$. In our case we have $\left\langle\delta_{\mathcal{M}} \mathcal{T} \mid T_{2}\right\rangle$, and since the bra is projected, the pseudovector part of $T_{2}$ drops out giving us the equation $\left(T_{2}\right)_{M N}=0$. If the tensor $T_{2}$ on the ket is itself divergenceless, then it also follows that $\hat{T}_{2}=0$, and thus simply that $T_{2}=0$. This is the case for us, so the field equations following from the $\delta_{\mathcal{M}}$ variation is

$$
\mathcal{T} \star \mathcal{T}=2 \mathcal{S}
$$

This implies our OPE field equation $\left(\mathcal{T} \circ_{2} \mathcal{T}-2 \mathcal{S}\right)^{M N}=0$, since all products are the same for the two-index part of the tensor.

Now we consider the $\phi$ variation. This is more subtle because the projection uses $\mathcal{S}$, which contains $\phi$, so the projection itself is varied. Consider an arbitrary tensor $T$ and its constrained projection $\bar{T}$. Using (5.33) we have that the variation of the projection is not projected

$$
\delta \bar{T}=\overline{\delta T}-\frac{1}{2}\left(\delta_{\phi} \operatorname{div} T\right)^{\prime}+\frac{1}{4}\left(\delta_{\phi} \operatorname{div}(\operatorname{div} T)\right)^{\prime \prime}+\frac{1}{4}\left(\operatorname{div}\left(\delta_{\phi} \operatorname{div} T\right)\right)^{\prime \prime} .
$$

While we could proceed without calculating the doubly primed terms (which will drop out) it is of interest to obtain a general formula for the variation of the projection. Taking dilaton variations of the products $\mathcal{S} \circ T$ and $\mathcal{S} \circ V$ one quickly shows that

$$
\delta_{\phi} \operatorname{tr} T=-6\langle\delta \phi \mid T\rangle, \quad \delta_{\phi} \operatorname{div} T=-\delta \phi \circ_{1} T, \quad \delta_{\phi} \operatorname{div} V=\langle V \mid \delta \phi\rangle,
$$

giving us

$$
\delta \bar{T}=\overline{\delta T}+\frac{1}{2}\left(\delta \phi \circ_{1} T\right)^{\prime}+\frac{1}{4}\left(\langle\operatorname{div} T \mid \delta \phi\rangle-\operatorname{div}\left(\delta \phi \circ_{1} T\right)\right)^{\prime \prime} .
$$

Since $\hat{T}_{M}$ drops out of $\bar{T}$, and thus from the full variation $\delta \bar{T}$, it is possible to rewrite the above right-hand side solely in terms of $\bar{T}$. For this we note that

$$
\delta \phi \circ_{1} T=\delta \phi \circ_{1} \bar{T}+\frac{1}{2} \delta \phi \circ_{1}(\operatorname{div} T)^{\prime}-\frac{1}{4} \delta \phi \circ_{1}(\operatorname{div} \operatorname{div} T)^{\prime \prime} .
$$

The last term vanishes by repeated use of the symmetry and derivative identities $\left(f \circ_{1} V^{\prime}=\right.$ $-\langle V \mid f\rangle^{\prime}$ which then implies $\left.f_{1} \circ_{1} f^{\prime \prime}=0\right)$, and we get

$$
\delta \phi \circ_{1} T=\delta \phi \circ_{1} \bar{T}-\frac{1}{2}\langle\operatorname{div} T \mid \delta \phi\rangle^{\prime} .
$$

Using this identity twice in (6.45) as well as (6.17) we finally find the desired variation formula:

$$
\delta \bar{T}=\overline{\delta T}+\frac{1}{2}\left(\delta \phi \circ_{1} \bar{T}\right)^{\prime}+\frac{1}{2}\langle\delta \phi \mid \bar{T}\rangle^{\prime \prime} .
$$

Applied to our divergence free $\mathcal{T}$ it reads

$$
\delta \mathcal{T}=\overline{\delta \mathcal{T}}+\frac{1}{2}\left(\delta \phi \circ_{1} \mathcal{T}\right)^{\prime}+\frac{1}{2}\langle\delta \phi \mid \mathcal{T}\rangle^{\prime \prime},
$$


The first term is the $\delta_{\mathcal{M}}$ we have already evaluated; we now consider only the latter two terms that comprise the $\delta_{\phi} \mathcal{T}$ variation

$$
\delta_{\phi} \mathcal{T}=\frac{1}{2}\left(\delta \phi \circ_{1} \mathcal{T}\right)^{\prime}+\frac{1}{2}\langle\delta \phi \mid \mathcal{T}\rangle^{\prime \prime} .
$$

The action is of the form

$$
\int e^{\phi}\left\langle\bar{T}_{1} \mid \bar{T}_{2}\right\rangle, \quad \bar{T}_{1}=\mathcal{T}, \quad \bar{T}_{2}=\mathcal{S}-\frac{1}{6} \mathcal{T} \star \mathcal{T} .
$$

From the above variation we see, since $\left\langle V^{\prime} \mid \bar{T}\right\rangle=\left\langle\bar{T} \mid V^{\prime}\right\rangle \sim 0$ in the integral, that the variation of the projection operator on $T_{1}$ or $T_{2}$ will vanish, as the integral of a total derivative. (In particular, $\delta \mathcal{S}=-\frac{1}{2}(\delta \phi)^{\prime \prime}$.) However, $\mathcal{T} \star \mathcal{T}$ is itself the projection of $\mathcal{T} \mathrm{O}_{2} \mathcal{T}$ : so we can ignore the projection to get $\star$ from $\mathrm{o}_{2}$, but not the projections implicit in the $\mathcal{T}$ 's inside the product: in fact, these are the only $\delta_{\phi}$ 's that contribute to the action, other than that of the measure $e^{\phi}$. We thus find

$$
\delta_{\phi} S=\int e^{\phi}\left[(\delta \phi) L-\frac{1}{3}\left\langle\mathcal{T} \mid \delta_{\phi} \mathcal{T} \star \mathcal{T}\right\rangle\right]
$$

where we have noted that the variations $\delta_{\phi} \mathcal{T}$ on bras are total derivatives, and applied the symmetry of the $\star$ product. The result is then

$$
\delta_{\phi} S=\int e^{\phi}\left[(\delta \phi) L+\frac{1}{6}\left\langle\mathcal{T} \mid\left(\delta \phi \circ_{1} \mathcal{T}\right) \star \mathcal{T}\right\rangle\right]
$$

where the double derivative term does not contribute because of the derivative identity $f^{\prime \prime} \circ_{2} T=0$.

From the distributive identity with $\mathcal{O}_{1}=V, \mathcal{O}_{2}=\bar{T}, \mathcal{O}_{3}=\bar{T}$, and $w=2, w^{\prime}=3$ we find

$$
\left\langle\bar{T} \mid V \circ_{2} \bar{T}\right\rangle=\left\langle V \mid \bar{T} \circ_{2} \bar{T}\right\rangle-2\left\langle\bar{T} \mid V \circ_{1} \bar{T}\right\rangle-2\langle\bar{T} \mid\langle V \mid \bar{T}\rangle\rangle .
$$

Because both vectors or scalars contracted with projected tensors are total derivatives under $\int e^{\phi}$, the last two terms in the above right-hand side can be dropped:

$$
\left\langle\bar{T} \mid V \circ_{2} \bar{T}\right\rangle \sim\left\langle V \mid \bar{T} \circ_{2} \bar{T}\right\rangle .
$$

Using (6.33) for the above right-hand side, we then have

$$
\left\langle\bar{T} \mid V \circ_{2} \bar{T}\right\rangle \sim \frac{1}{4}\left\langle V \mid\langle\bar{T} \mid \bar{T}\rangle^{\prime \prime}\right\rangle \sim \frac{1}{2}\langle V \mid\langle\bar{T} \mid \bar{T}\rangle\rangle,
$$

where the last step used the derivative identity and the symmetry property twice. Applying this to $\bar{T}=\mathcal{T}$ we get

$$
\left\langle\mathcal{T} \mid V \circ_{2} \mathcal{T}\right\rangle \sim \frac{1}{2}\langle V \mid\langle\mathcal{T} \mid \mathcal{T}\rangle\rangle .
$$

For further simplification, we use the first equation in (6.8), applied to $\mathcal{T}$ to get

$$
\operatorname{tr}(\mathcal{T} \star \mathcal{T})=2\langle\operatorname{tr} \mathcal{T} \mid \mathcal{T}\rangle+4\langle\mathcal{T} \mid \mathcal{T}\rangle
$$


By the double metric equation of motion, the left hand side is a constant. Under a derivative we can therefore replace $\langle\mathcal{T} \mid \mathcal{T}\rangle$ by $-\frac{1}{2}\langle\operatorname{tr} \mathcal{T} \mid \mathcal{T}\rangle$. Indeed, using $\langle V \mid f\rangle=V^{M} \partial_{M} f$, we see that $\langle\mathcal{T} \mid \mathcal{T}\rangle$ appears under a derivative in (6.57) and therefore,

$$
\left\langle\mathcal{T} \mid V \circ_{2} \mathcal{T}\right\rangle \sim-\frac{1}{4}\langle V \mid\langle\mathcal{T} \mid \operatorname{tr} \mathcal{T}\rangle\rangle
$$

With this result we can return to our variation (6.53), identify $V=\delta \phi \circ_{1} \mathcal{T}$ and then obtain

$$
\delta_{\phi} S=\int e^{\phi}\left[(\delta \phi) \frac{1}{3} \operatorname{tr} \mathcal{T}-\frac{1}{24}\left\langle\delta \phi \circ_{1} \mathcal{T} \mid\langle\mathcal{T} \mid \operatorname{tr} \mathcal{T}\rangle\right\rangle\right]
$$

where we simplified $L$ by using the double metric equation of motion.

In order to isolate the $\delta \phi$ factor in the second term, we consider another distributive identity $\left(\mathcal{O}_{1}=\mathcal{S}, \mathcal{O}_{2}=f_{2}, \mathcal{O}_{3}=V\right.$ with $\left.\hat{w}=0, w=0, w^{\prime}=3\right)$

$$
\operatorname{div}\left(f_{2} \circ_{1} V\right)=\left\langle f_{2} \mid \operatorname{div} V\right\rangle-\left\langle f_{2} \mid V\right\rangle \quad \rightarrow \quad\left\langle V \mid f_{2}\right\rangle \sim-\left\langle f_{2} \mid \operatorname{div} V\right\rangle
$$

Taking now $V=f_{1} \circ_{1} \bar{T}$ and noting that $\operatorname{div}\left(f_{1} \circ_{1} \bar{T}\right)=-2\left\langle f_{1} \mid \bar{T}\right\rangle$ (see (6.18)), we have

$$
\left\langle f_{1} \circ_{1} \bar{T} \mid f_{2}\right\rangle \sim 2\left\langle f_{2} \mid\left\langle f_{1} \mid \bar{T}\right\rangle\right\rangle
$$

The distributive identity $\left(\mathcal{O}_{1}=f_{1}, \mathcal{O}_{2}=f_{2}, \mathcal{O}_{3}=T\right.$ with $\left.\hat{w}=0, w=w^{\prime}=1\right)$

$$
\left\langle f_{1} \mid f_{2} \circ_{1} T\right\rangle-\left\langle f_{2} \mid f_{1} \circ_{1} T\right\rangle=0
$$

informs us that the left-hand side of (6.62) is symmetric under the exchange of the two functions, so that we have

$$
\left\langle f_{1} \circ_{1} \bar{T} \mid f_{2}\right\rangle \sim 2\left\langle f_{1} \mid\left\langle f_{2} \mid \bar{T}\right\rangle\right\rangle=2 f_{1}\left\langle f_{2} \mid \bar{T}\right\rangle
$$

where in the last equality we noted that the inner product of two functions is equal to their ordinary product (by the strong constraint there are no contractions in the operator product of two functions). This is our desired result. With the relevant choices of $f$ 's and tensor it reads:

$$
\left\langle\delta \phi \circ_{1} \mathcal{T} \mid\langle\mathcal{T} \mid \operatorname{tr} \mathcal{T}\rangle\right\rangle=2 \delta \phi\langle\mathcal{T} \mid\langle\mathcal{T} \mid \operatorname{tr} \mathcal{T}\rangle\rangle
$$

Back to the action variation (6.60), we can finally rewrite the second term with $\delta \phi$ separated out:

$$
\delta_{\phi} S=\frac{1}{3} \int e^{\phi}(\delta \phi)\left[\operatorname{tr} \mathcal{T}-\frac{1}{4}\langle\mathcal{T} \mid\langle\mathcal{T} \mid \operatorname{tr} \mathcal{T}\rangle\rangle\right]
$$

The result is that variation of $\phi$ gives an operator of the form $I+\ldots$ acting on $\operatorname{tr} \mathcal{T}$, so the $\phi$ field equation is the expected $\operatorname{tr} \mathcal{T}=0$. 


\subsection{Field equation evaluation}

We'll need some explicit evaluations of operator products of tensors. For two equal tensors the OPE gives

$$
\begin{aligned}
\langle T \mid T\rangle= & \frac{1}{2} T^{P Q} T_{P Q}-\partial_{P} T^{L K} \partial_{L} T_{K}^{P}+\frac{1}{4} \partial_{P} \partial_{Q} T^{K L} \partial_{K} \partial_{L} T^{P Q} \\
& -\frac{3}{2}\langle\hat{T} \mid \hat{T}\rangle-3 \partial_{P} \hat{T}^{K} T_{K}^{P}+\frac{3}{2} \partial_{P} \partial_{Q} \hat{T}^{K} \partial_{K} T^{P Q} \\
\left(T \circ_{2} T\right)_{M N}=\{ & T, T\}_{M N}-\frac{1}{2} \partial_{M} T^{P Q} \partial_{N} T_{P Q}+T^{P Q} \partial_{P} \partial_{Q} T_{M N} \\
& +2 \partial_{(N} T^{L K} \partial_{L} T_{M) K}-2 \partial_{Q} T_{M}^{P} \partial_{P} T_{N}{ }^{Q} \\
& +\partial_{M} \partial_{P} T^{L K} \partial_{N} \partial_{L} T_{K}^{P}-\partial_{(N} \partial_{K} T^{P Q} \partial_{P} \partial_{Q} T^{K}{ }_{M)} \\
& -\frac{1}{4} \partial_{M} \partial_{P} \partial_{Q} T^{K L} \partial_{N} \partial_{K} \partial_{L} T^{P Q} \\
& +\hat{T}^{K} \partial_{K} T_{M N}+\left(\partial_{(N} \hat{T}^{K}-\partial^{K} \hat{T}_{(N}\right) T_{M) K}-\partial_{(N} \partial_{P} \hat{T}^{K} \partial_{K} T^{P}{ }_{M)} \\
& +\frac{1}{2} \partial_{P}\left(\partial_{(M} \hat{T}_{Q}-\partial_{Q} \hat{T}_{(M}\right) \partial_{N)} T^{P Q}-\frac{1}{4} \partial_{(M} \partial_{P} \partial_{Q} \hat{T}^{K} \partial_{N)} \partial_{K} T^{P Q}
\end{aligned}
$$

where $\left\langle\hat{T}_{1} \mid \hat{T}_{2}\right\rangle$ means the inner product of two pseudovectors $\hat{T}^{M} Z_{M}$ treated as if they were vectors.

Note that the above imply the corresponding results for two different tensors, since for any bilinear product $\diamond$ we have

$$
\mathcal{O}_{(1} \diamond \mathcal{O}_{2)}=\left(\mathcal{O}_{1}+\mathcal{O}_{2}\right) \diamond\left(\mathcal{O}_{1}+\mathcal{O}_{2}\right)-\mathcal{O}_{1} \diamond \mathcal{O}_{1}-\mathcal{O}_{2} \diamond \mathcal{O}_{2}
$$

In practice, this means to just substitute $T_{1}$ and $T_{2}$ for the two $T$ 's in each term in the above equations in the two possible ways, then average to get $\left\langle T_{1} \mid T_{2}\right\rangle$ and $\left(T_{1} \circ_{2} T_{2}\right)_{M N}$. Note that $\left(T_{1} \circ_{2} T_{2}\right)_{M N}$ is symmetric under $1 \leftrightarrow 2$ because the lack of symmetry in $T_{1} \circ_{2} T_{2}$ only affects the pseudovector part.

Our full double-metric field equation $\left(\mathcal{M} \circ_{2} \mathcal{M}\right)_{M N}=2 \eta_{M N}$ is therefore

$$
\begin{aligned}
\left(\mathcal{M}^{2}\right)_{M N}= & \eta_{M N}+\frac{1}{4} \partial_{M} \mathcal{M}^{P Q} \partial_{N} \mathcal{M}_{P Q}-\frac{1}{2} \mathcal{M}^{P Q} \partial_{P} \partial_{Q} \mathcal{M}_{M N} \\
& -\partial_{(N} \mathcal{M}^{L K} \partial_{L} \mathcal{M}_{M) K}+\partial_{Q} \mathcal{M}_{M}{ }^{P} \partial_{P} \mathcal{M}_{N}{ }^{Q} \\
& -\frac{1}{2} \partial_{M} \partial_{P} \mathcal{M}^{L K} \partial_{N} \partial_{L} \mathcal{M}_{K}{ }^{P}+\frac{1}{2} \partial_{(N} \partial_{K} \mathcal{M}^{P Q} \partial_{P} \partial_{Q} \mathcal{M}^{K}{ }_{M)} \\
& +\frac{1}{8} \partial_{M} \partial_{P} \partial_{Q} \mathcal{M}^{K L} \partial_{N} \partial_{K} \partial_{L} \mathcal{M}^{P Q} \\
& -\frac{1}{2} G^{K} \partial_{K} \mathcal{M}_{M N}-\frac{1}{2}\left(\partial_{(N} G^{K}-\partial^{K} G_{(N}\right) \mathcal{M}_{M) K}+\frac{1}{2} \partial_{(N} \partial_{P} G^{K} \partial_{K} \mathcal{M}^{P}{ }_{M)} \\
& -\frac{1}{4} \partial_{P}\left(\partial_{(M} G_{Q}-\partial_{Q} G_{(M}\right) \partial_{N)} \mathcal{M}^{P Q}+\frac{1}{8} \partial_{(M} \partial_{P} \partial_{Q} G^{K} \partial_{N)} \partial_{K} \mathcal{M}^{P Q} .
\end{aligned}
$$

where $G^{M}=G^{M}(\mathcal{M}, \phi)$, as defined in (5.37). While $G^{M}$ has terms with one derivative and terms with three derivatives, the latter carry the index on a derivative $\partial^{M}$ and cannot 
contribute in the last term. The equation of motion has terms with zero, two, four, and six derivatives. There cannot be terms with more than six derivatives since the strong constraint does not allow one to write any such terms.

\section{Relation to generalized metric formulation}

In this section we will relate our formalism to the generalized metric. In particular, we confirm that for the two-derivative approximation the field equations reduce to the known double field theory equations in terms of the generalized metric $\mathcal{H}_{M N}$ and the dilaton [18]. We review the $\mathcal{H}$ equation and show that it arises from the $\mathcal{M}$ field equation. Then we show that $\operatorname{tr}(\mathcal{T})$ reproduces the generalized curvature scalar $\mathcal{R}(\mathcal{H}, \phi)$, which encodes the dilaton equation.

\subsection{Classical action}

Consider the action $S=\int e^{\phi} L$ with $L(\mathcal{M})$ a Lagrangian for an arbitrary matrix $\mathcal{M}_{M N}$, whose indices are raised and lowered with $\eta^{M N}$ :

$$
L=\frac{1}{8} \mathcal{M}^{M N} \partial_{M} \mathcal{M}^{K L} \partial_{N} \mathcal{M}_{K L}-\frac{1}{2} \mathcal{M}^{M N} \partial_{N} \mathcal{M}^{K L} \partial_{L} \mathcal{M}_{M K}-\mathcal{M}^{M N} \partial_{M} \partial_{N} \phi .
$$

If we were to set $\mathcal{M}$ equal to the (constrained) generalized metric $\mathcal{H}$, the resulting $L$ is the simplest form of the double field theory Lagrangian of [18]. This connection requires the identification

$$
\phi=-2 d .
$$

Varying with respect to the unconstrained $\mathcal{M}$ we find

$$
\delta_{\mathcal{M}} S=\int e^{\phi} \delta_{\mathcal{M}} L, \quad \delta_{\mathcal{M}} L=\delta \mathcal{M}^{M N} \mathcal{K}_{M N}(\mathcal{M}),
$$

where

$$
\begin{aligned}
\mathcal{K}_{M N}(\mathcal{M}) \equiv & \frac{1}{8} \partial_{M} \mathcal{M}^{K L} \partial_{N} \mathcal{M}_{K L}-\frac{1}{4}\left(\partial_{L}+\partial_{L} \phi\right)\left(\mathcal{M}^{L K} \partial_{K} \mathcal{M}_{M N}\right)-\partial_{M} \partial_{N} \phi \\
& -\frac{1}{4} \partial_{(M} \mathcal{M}^{K L} \partial_{L} \mathcal{M}_{N) K}+\frac{1}{4}\left(\partial_{L}+\partial_{L} \phi\right)\left(\mathcal{M}^{K L} \partial_{(M} \mathcal{M}_{N) K}+\mathcal{M}^{K}{ }_{(M} \partial_{K} \mathcal{M}^{L}{ }_{N)}\right)
\end{aligned}
$$

It is convenient to rewrite this expression in terms of the pseudovector part of $\mathcal{T}$,

$$
G^{M}(\mathcal{M}, \phi)=\partial_{L} \mathcal{M}^{L K}+\partial_{L} \phi \mathcal{M}^{L K}+\cdots,
$$

leaving out higher-derivative terms in (5.37) that are irrelevant for our present purposes. One finds

$$
\begin{aligned}
\mathcal{K}_{M N} \equiv & \frac{1}{8} \partial_{M} \mathcal{M}^{K L} \partial_{N} \mathcal{M}_{K L}-\frac{1}{4} \mathcal{M}^{L K} \partial_{K} \partial_{L} \mathcal{M}_{M N}-\frac{1}{4} G^{K} \partial_{K} \mathcal{M}_{M N}-\partial_{M} \partial_{N} \phi \\
& -\frac{1}{4} \partial_{(M} \mathcal{M}^{K L} \partial_{L} \mathcal{M}_{N) K}+\frac{1}{4} \mathcal{M}^{K L} \partial_{(M} \partial_{L} \mathcal{M}_{N) K}+\frac{1}{4} \partial_{L} \mathcal{M}_{(M}{ }^{K} \partial_{K} \mathcal{M}_{N)}{ }^{L} \\
& +\frac{1}{4} G^{K} \partial_{(M} \mathcal{M}_{N) K}+\frac{1}{4} \mathcal{M}^{K}{ }_{(M} \partial_{K} G_{N)}-\frac{1}{2} \mathcal{M}^{K}{ }_{M} \mathcal{M}^{L}{ }_{N} \partial_{K} \partial_{L} \phi
\end{aligned}
$$


It is useful to note that the above variation (7.3) also applies to the equivalent form $L^{\prime}$ of the Lagrangian that yields the same action as $L$ :

$$
\begin{aligned}
L^{\prime}=\mathcal{R}(\mathcal{M}, \phi) \equiv & \frac{1}{8} \mathcal{M}^{M N} \partial_{M} \mathcal{M}^{K L} \partial_{N} \mathcal{M}_{K L}-\frac{1}{2} \mathcal{M}^{M N} \partial_{N} \mathcal{M}^{K L} \partial_{L} \mathcal{M}_{M K} \\
& -2 \mathcal{M}^{M N} \partial_{M} \partial_{N} \phi-\partial_{M} \partial_{N} \mathcal{M}^{M N}-\mathcal{M}^{M N} \partial_{M} \phi \partial_{N} \phi-2 \partial_{M} \mathcal{M}^{M N} \partial_{N} \phi
\end{aligned}
$$

Note that $\mathcal{R}(\mathcal{H}, \phi)$ is the scalar curvature of [18]. The $\mathcal{K}(\mathcal{M})$ above also coincides with $\mathcal{K}(\mathcal{H})$ in [18] (eq. (4.49)), when $\mathcal{M}$ is replaced $\mathcal{H}$. Since $\mathcal{H}$ is a constrained field, its equation of motion is not the vanishing of $\mathcal{K}(\mathcal{H})$. Rather, the field equation is given by eq. (4.57) in [18] which, written out explicitly, reads

$$
\mathcal{K}(\mathcal{H})-\mathcal{H} \mathcal{K}(\mathcal{H}) \mathcal{H}=0
$$

Multiplying from the right with $\mathcal{H}$ and using $\mathcal{H}^{2}=1$ gives

$$
\mathcal{K}(\mathcal{H}) \mathcal{H}-\mathcal{H} \mathcal{K}(\mathcal{H})=0
$$

which is the form of the equation of motion that we will re-derive below.

\subsection{Double metric action and field equation}

Consider the action

$$
S=\int e^{\phi} L, \quad L=\frac{1}{2} \operatorname{tr}(\mathcal{T})-\frac{1}{6}\langle\mathcal{T} \mid \mathcal{T} \star \mathcal{T}\rangle
$$

to second order in derivatives. The Lagrangian can be easily computed, recalling that the $\star$ product projects onto divergence-free tensors, so that the pseudovector part of a star product is given by

$$
\left(T_{1} \star T_{2}\right)^{M}=G^{M}\left(\left(T_{1} \circ_{2} T_{2}\right)^{M N}, \phi\right),
$$

with the right-hand side defined in (5.37). The term $\operatorname{tr}(\mathcal{T})$ can be evaluated explicitly from the first equation in (5.17), using the determined expression $\hat{\mathcal{T}}^{M}=G^{M}\left(\mathcal{M}^{M N}, \phi\right)$. Similarly, the cubic term can be straightforwardly computed from the explicit form of the inner product and $\circ_{2}$ given in (6.67). One finds

$$
\begin{aligned}
L= & \frac{1}{2}\left[\operatorname{Tr}\left(\mathcal{M}-\frac{1}{3} \mathcal{M}^{3}\right)-3 \mathcal{M}^{M N} \partial_{M} \partial_{N} \phi-3 \partial_{N} G^{N}(\mathcal{M})-3 \partial_{N} \phi G^{N}(\mathcal{M})\right. \\
& +\frac{1}{12} \mathcal{M}^{M N} \partial_{M} \mathcal{M}^{P Q} \partial_{N} \mathcal{M}_{P Q}-\frac{1}{6} \mathcal{M}^{M N} \mathcal{M}^{P Q} \partial_{P} \partial_{Q} \mathcal{M}_{M N} \\
& -\frac{2}{3} \mathcal{M}^{M N} \partial_{M} \mathcal{M}^{L K} \partial_{L} \mathcal{M}_{K N}+\frac{1}{3} \mathcal{M}^{M N} \partial_{Q} \mathcal{M}_{M}{ }^{P} \partial_{P} \mathcal{M}_{N}{ }^{Q}+\frac{2}{3} \partial_{P} \mathcal{M}^{L K} \partial_{L}\left(\mathcal{M}^{2}\right)_{K}{ }^{P} \\
& -\frac{1}{6} \mathcal{M}^{M N} G^{K}(\mathcal{M}) \partial_{K} \mathcal{M}_{M N}+\partial_{P} G^{K}(\mathcal{M})\left(\mathcal{M}^{2}\right)_{K}{ }^{P} \\
& \left.+G^{M}(\mathcal{M}) G_{M}\left(\mathcal{M}^{2}\right)+\partial_{P} G^{K}\left(\mathcal{M}^{2}\right) \mathcal{M}_{K}{ }^{P}\right] .
\end{aligned}
$$


For brevity we have dropped the dilaton input from $G(\mathcal{M}, \phi)$ and $G\left(\mathcal{M}^{2}, \phi\right)$. It is now a straightforward though somewhat tedious calculation to verify that, up to total derivatives, the corresponding action reads

$$
\begin{aligned}
S= & \int e^{\phi}\left[\frac{1}{2} \eta^{M N}\left(\mathcal{M}-\frac{1}{3} \mathcal{M}^{3}\right)_{M N}+\frac{1}{2}\left(\mathcal{M}^{2}-1\right)^{M P} \mathcal{M}_{P}{ }^{N} \partial_{M} \partial_{N} \phi\right. \\
& \left.+\frac{1}{8} \mathcal{M}^{M N} \partial_{M} \mathcal{M}^{P Q} \partial_{N} \mathcal{M}_{P Q}-\frac{1}{2} \mathcal{M}^{M N} \partial_{N} \mathcal{M}^{K L} \partial_{L} \mathcal{M}_{K M}-\mathcal{M}^{M N} \partial_{M} \partial_{N} \phi\right]
\end{aligned}
$$

Since the last line coincides with (7.1), its variation equals $\mathcal{K}_{M N}$ determined in (7.4). Thus, the total variation with respect to $\mathcal{M}$ is given by

$0=\frac{1}{2}\left(\eta-\mathcal{M}^{2}\right)_{M N}+\frac{1}{2}\left(\mathcal{M}^{2}\right)_{(M}{ }^{K} \partial_{N)} \partial_{K} \phi+\frac{1}{2} \mathcal{M}_{M}{ }^{P} \mathcal{M}_{N}{ }^{Q} \partial_{P} \partial_{Q} \phi-\frac{1}{2} \partial_{M} \partial_{N} \phi+\mathcal{K}_{M N}(\mathcal{M})$.

Using the zeroth-order relation $\mathcal{M}^{2}=1$ in the second term we find

$$
0=\frac{1}{2}\left(\eta-\mathcal{M}^{2}\right)_{M N}+\frac{1}{2} \mathcal{M}_{M}^{P} \mathcal{M}_{N}{ }^{Q} \partial_{P} \partial_{Q} \phi+\frac{1}{2} \partial_{M} \partial_{N} \phi+\mathcal{K}_{M N}(\mathcal{M})
$$

Let us now show that this equation coming from the double metric action is the $\mathcal{T}$ equation $(\mathcal{T} \star \mathcal{T})_{M N}=2 \eta_{M N}$ from the OPE. We rewrite this in matrix notation as

$$
\mathcal{M}^{2}=1+2 \mathcal{V}(\mathcal{M}) \rightarrow \frac{1}{2}\left(1-\mathcal{M}^{2}\right)+\mathcal{V}(\mathcal{M})=0 .
$$

Equation (6.69) allows us to identify the two-derivative part $\mathcal{V}^{(2)}$ of $\mathcal{V}$ as

$$
\begin{aligned}
\mathcal{V}_{M N}^{(2)}(\mathcal{M})= & \frac{1}{8} \partial_{M} \mathcal{M}^{P Q} \partial_{N} \mathcal{M}_{P Q}-\frac{1}{4} \mathcal{M}^{P Q} \partial_{P} \partial_{Q} \mathcal{M}_{M N}-\frac{1}{2} \partial_{(M} \mathcal{M}^{K L} \partial_{L} \mathcal{M}_{N) K} \\
& +\frac{1}{2} \partial_{Q} \mathcal{M}_{M}{ }^{P} \partial_{P} \mathcal{M}_{N}{ }^{Q}-\frac{1}{4} G^{K} \partial_{K} \mathcal{M}_{M N}-\frac{1}{4}\left(\partial_{(M} G^{K}-\partial^{K} G_{(M}\right) \mathcal{M}_{N) K}
\end{aligned}
$$

where only the parts of $G^{K}$ with one derivative are included. Next we have to relate $\mathcal{V}_{M N}^{(2)}$ to $\mathcal{K}_{M N}$. Using (7.6) we find

$$
\begin{aligned}
\mathcal{V}_{M N}^{(2)}(\mathcal{M})= & \mathcal{K}_{M N}(\mathcal{M})-\frac{1}{4} G^{K} \partial_{(M} \mathcal{M}_{N) K}-\frac{1}{4} \partial_{(N} G^{K} \mathcal{M}_{M) K}-\frac{1}{4} \partial_{(M} \mathcal{M}^{K L} \partial_{L} \mathcal{M}_{N) K} \\
& -\frac{1}{4} \mathcal{M}^{K L} \partial_{L} \partial_{(M} \mathcal{M}_{N) K}+\partial_{M} \partial_{N} \phi+\frac{1}{2} \mathcal{M}^{K}{ }_{M} \mathcal{M}^{L}{ }_{N} \partial_{K} \partial_{L} \phi
\end{aligned}
$$

We now use $\mathcal{M}^{2}=1$ in the two-derivative terms, which implies in particular

$$
-\frac{1}{2} \mathcal{M}^{K L} \partial_{M} \mathcal{M}_{N K}=\frac{1}{2} \mathcal{M}_{N K} \partial_{M} \mathcal{M}^{K L}
$$


Acting here with $\partial_{L}$ implies

$$
\begin{aligned}
-\frac{1}{2} \mathcal{M}^{K L} \partial_{L} \partial_{M} \mathcal{M}_{N K}= & \frac{1}{2} \partial_{L} \mathcal{M}^{L K} \partial_{M} \mathcal{M}_{N K}+\frac{1}{2} \partial_{L} \mathcal{M}_{N K} \partial_{M} \mathcal{M}^{K L}+\frac{1}{2} \mathcal{M}_{N K} \partial_{M} \partial_{L} \mathcal{M}^{L K} \\
= & \frac{1}{2} G^{K} \partial_{M} \mathcal{M}_{N K}-\frac{1}{2} \partial_{L} \phi \mathcal{M}^{L K} \partial_{M} \mathcal{M}_{N K}+\frac{1}{2} \partial_{M} \mathcal{M}^{K L} \partial_{L} \mathcal{M}_{N K} \\
& +\frac{1}{2} \mathcal{M}_{N K} \partial_{M} G^{K}-\frac{1}{2} \mathcal{M}_{N K} \partial_{M}\left(\partial_{L} \phi \mathcal{M}^{L K}\right) \\
= & \frac{1}{2} G^{K} \partial_{M} \mathcal{M}_{N K}+\frac{1}{2} \partial_{M} \mathcal{M}^{K L} \partial_{L} \mathcal{M}_{N K} \\
& +\frac{1}{2} \mathcal{M}_{N K} \partial_{M} G^{K}-\frac{1}{2} \partial_{M} \partial_{N} \phi
\end{aligned}
$$

Using this in (7.18) many terms cancel and we finally get

$$
\mathcal{V}_{M N}^{(2)}(\mathcal{M})=\mathcal{K}_{M N}(\mathcal{M})+\frac{1}{2} \partial_{M} \partial_{N} \phi+\frac{1}{2} \mathcal{M}^{K}{ }_{M} \mathcal{M}^{L}{ }_{N} \partial_{K} \partial_{L} \phi
$$

Inserting this in (7.16) we obtain

$$
0=\frac{1}{2}\left(\eta-\mathcal{M}^{2}\right)_{M N}+\mathcal{K}_{M N}(\mathcal{M})+\frac{1}{2} \partial_{M} \partial_{N} \phi+\frac{1}{2} \mathcal{M}^{K}{ }_{M} \mathcal{M}^{L}{ }_{N} \partial_{K} \partial_{L} \phi
$$

which is in perfect agreement with (7.15), as we wanted to show.

We will now show that (7.16) implies the equation of motion and the constraint for the generalized metric $\mathcal{H}$. Indeed, multiplying by $\mathcal{M}$ from the left and subtracting the same equation but multiplied by $\mathcal{M}$ from the right we quickly see that

$$
\mathcal{V}(\mathcal{M}) \mathcal{M}-\mathcal{M V}(\mathcal{M})=0
$$

Next we do an $\alpha^{\prime}$ expansion by writing

$$
\mathcal{M}=\mathcal{H}+\Lambda(\mathcal{H}), \quad \text { with } \mathcal{H}^{2}=1,
$$

where $\Lambda(\mathcal{H})$ is first order in $\alpha^{\prime}$, containing two derivatives. To leading order (7.23) gives

$$
\mathcal{V}^{(2)}(\mathcal{H}) \mathcal{H}-\mathcal{H} \mathcal{V}^{(2)}(\mathcal{H})=0
$$

We quickly confirm that the difference in $(7.21)$ between $\mathcal{K}$ and $\mathcal{V}^{(2)}$ drops out from the above field equation. Thus,

$$
\mathcal{V}^{(2)}(\mathcal{H}) \mathcal{H}-\mathcal{H} \mathcal{V}^{(2)}(\mathcal{H})=\mathcal{K}(\mathcal{H}) \mathcal{H}-\mathcal{H} \mathcal{K}(\mathcal{H})=0
$$

The last equality is the field equation (7.9) for $\mathcal{H}$ in DFT. We have reproduced it correctly from the double-metric.

We now determine $\Lambda(\mathcal{H})$ in (7.24). Using the expansion in (7.16) gives

$$
\mathcal{H} \Lambda(\mathcal{H})+\Lambda(\mathcal{H}) \mathcal{H}=2 \mathcal{V}^{(2)}(\mathcal{H}) .
$$

This equation also contains the field equation (7.25): it is obtained by multiplying the above by $\mathcal{H}$ from the left, and subtracting the equation in which we multiply by $\mathcal{H}$ from 
the right. On the other hand solving for $\Lambda$ from (7.27) looks at first sight impossible, since it would appear to trivialize $\mathcal{V}^{(2)}$. But this is not the case if the solution involves the field equation. Indeed we can take

$$
\Lambda=\frac{1}{2}\left\{\mathcal{H}, \mathcal{V}^{(2)}(\mathcal{H})\right\}
$$

Then back on the left-hand side of (7.27) and using $\mathcal{H}^{2}=1$ we get

$$
\mathcal{V}^{(2)}+\mathcal{H} \mathcal{V}^{(2)} \mathcal{H}=2 \mathcal{V}^{(2)}+\mathcal{H}\left[\mathcal{V}^{(2)}, \mathcal{H}\right]=2 \mathcal{V}^{(2)},
$$

where in the last step we used the equation of motion. So we can write,

$$
\mathcal{M}(\mathcal{H})=\mathcal{H}+\frac{1}{2}\left\{\mathcal{H}, \mathcal{V}^{(2)}(\mathcal{H})\right\}+\mathcal{O}\left(\alpha^{2}\right)
$$

We note that this parameterization of $\mathcal{M}$ in terms of $\mathcal{H}$ has assumed the equation of motion for $\mathcal{H}$. It can therefore be used in the form $\mathcal{M}(\mathcal{H})=\mathcal{H}+\mathcal{H V}^{(2)}$.

\subsection{Dilaton equation}

We now analyze the dilaton equation

$$
\operatorname{tr}(\mathcal{T})=\eta^{M N} \mathcal{M}_{M N}-3 \partial_{M} \partial_{N} \mathcal{H}^{M N}-6 \mathcal{H}^{M N} \partial_{M} \partial_{N} \phi-6 \partial_{M} \mathcal{H}^{M N} \partial_{N} \phi-3 \mathcal{H}^{M N} \partial_{M} \phi \partial_{N} \phi,
$$

where we were allowed to replace $\mathcal{M}=\mathcal{H}$ in the $\mathcal{O}\left(\alpha^{\prime}\right)$ term. We will show that, in the two-derivative approximation, it gives rise to the scalar curvature of double field theory when written in terms of the generalized metric $\mathcal{H}$, and thus to the usual dilaton equation.

First, we insert (7.28),

$$
\eta^{M N} \mathcal{M}_{M N}=\eta^{M N} \Lambda_{M N}(\mathcal{H})=\mathcal{H}^{M N} \mathcal{V}_{M N}
$$

The tensor $\mathcal{V}^{(2)}$ is given in (7.17),

$$
\begin{aligned}
\mathcal{V}_{M N}^{(2)}= & \frac{1}{8} \partial_{M} \mathcal{H}^{P Q} \partial_{N} \mathcal{H}_{P Q}-\frac{1}{4} \mathcal{H}^{P Q} \partial_{P} \partial_{Q} \mathcal{H}_{M N}-\frac{1}{2} \partial_{(M} \mathcal{H}^{K L} \partial_{L} \mathcal{H}_{N) K} \\
& +\frac{1}{2} \partial_{Q} \mathcal{H}_{M}{ }^{P} \partial_{P} \mathcal{H}_{N}{ }^{Q}-\frac{1}{4} G^{K} \partial_{K} \mathcal{H}_{M N}-\frac{1}{4}\left(\partial_{(M} G^{K}-\partial^{K} G_{(M}\right) \mathcal{H}_{N) K},
\end{aligned}
$$

where we replaced everywhere $\mathcal{M}$ by $\mathcal{H}$. Thus,

$$
\begin{aligned}
\mathcal{H}^{M N} \mathcal{V}_{M N}^{(2)}=\frac{1}{8} \mathcal{H}^{M N} \partial_{M} \mathcal{H}^{K L} \partial_{N} \mathcal{H}_{K L}-\frac{1}{4} \mathcal{H}^{M N} \mathcal{H}^{P Q} \partial_{P} \partial_{Q} \mathcal{H}_{M N} \\
-\mathcal{H}^{M N} \partial_{M} \mathcal{H}^{K L} \partial_{L} \mathcal{H}_{N K}+\frac{1}{2} \mathcal{H}^{M N} \partial_{Q} \mathcal{H}_{M}{ }^{P} \partial_{P} \mathcal{H}_{N}{ }^{Q}
\end{aligned}
$$

A few terms dropped out by the constraint on $\mathcal{H}$, in particular all the $G$ terms. Using some identities following from $\mathcal{H}^{2}=1$,

$$
\begin{aligned}
-\frac{1}{4} \mathcal{H}^{K L} \mathcal{H}^{P Q} \partial_{K} \partial_{L} \mathcal{H}_{P Q} & =\frac{1}{4} \mathcal{H}^{K L} \partial_{K} \mathcal{H}^{P Q} \partial_{L} \mathcal{H}_{P Q}, \\
\frac{1}{2} \mathcal{H}^{P Q} \partial^{K} \mathcal{H}_{L P} \partial^{L} \mathcal{H}_{K Q} & =-\frac{1}{2} \mathcal{H}^{P L} \partial_{L} \mathcal{H}^{K Q} \partial_{K} \mathcal{H}_{P Q},
\end{aligned}
$$


one finds

$$
\eta^{M N} \mathcal{M}_{M N}=\mathcal{H}^{M N} \mathcal{V}_{M N}^{(2)}=3\left(\frac{1}{8} \mathcal{H}^{M N} \partial_{M} \mathcal{H}^{P Q} \partial_{N} \mathcal{H}_{P Q}-\frac{1}{2} \mathcal{H}^{M N} \partial_{M} \mathcal{H}^{K L} \partial_{L} \mathcal{H}_{N K}\right)
$$

Inserting now in (7.31) and re-expressing $\phi=-2 d$, we get

$$
\begin{aligned}
\operatorname{tr}(\mathcal{T})= & 3\left(\frac{1}{8} \mathcal{H}^{M N} \partial_{M} \mathcal{H}^{P Q} \partial_{N} \mathcal{H}_{P Q}-\frac{1}{2} \mathcal{H}^{M N} \partial_{M} \mathcal{H}^{K L} \partial_{L} \mathcal{H}_{N K}-\partial_{M} \partial_{N} \mathcal{H}^{M N}\right. \\
& \left.+4 \mathcal{H}^{M N} \partial_{M} \partial_{N} d+4 \partial_{M} \mathcal{H}^{M N} \partial_{N} d-4 \mathcal{H}^{M N} \partial_{M} d \partial_{N} d\right) \\
= & 3 \mathcal{R}(\mathcal{H}, d) .
\end{aligned}
$$

Thus, we get exactly the scalar curvature and so the dilaton field equation.

It is also instructive to verify that the equation $\operatorname{tr}(\mathcal{T})=0$ follows from the action $(7.10)$ upon using the $\mathcal{T}$ equation - instead of using the first-order solution of that equation. We thus vary (7.13) with respect to $\phi$, and we are allowed to use $\mathcal{M}^{2}=1$ in the two-derivative terms as a consequence of the $\mathcal{T}$ equation. We obtain

$$
\frac{1}{2} \operatorname{Tr}\left(\mathcal{M}-\frac{1}{3} \mathcal{M}^{3}\right)+\mathcal{R}(\mathcal{M}, \phi)=0 .
$$

Inserting now $\mathcal{M}^{2}=1+2 \mathcal{V}^{(2)}$ this becomes

$$
\operatorname{Tr} \mathcal{M}-\mathcal{M}^{M N} \mathcal{V}_{M N}^{(2)}+3 \mathcal{R}=0
$$

In $\mathcal{M}^{M N} \mathcal{V}_{M N}^{(2)}$ we may use $\mathcal{M}^{2}=1$, which with (7.34) yields

$$
-\mathcal{M}^{M N} \mathcal{V}_{M N}^{(2)}=-3\left(\frac{1}{8} \mathcal{M}^{M N} \partial_{M} \mathcal{M}^{P Q} \partial_{N} \mathcal{M}_{P Q}-\frac{1}{2} \mathcal{M}^{M N} \partial_{N} \mathcal{M}^{K L} \partial_{L} \mathcal{M}_{K M}\right)
$$

Back in (7.39) this gives

$\eta^{M N} \mathcal{M}_{M N}-6 \mathcal{M}^{M N} \partial_{M} \partial_{N} \phi-3 \partial_{M} \partial_{N} \mathcal{M}^{M N}-6 \partial_{M} \mathcal{M}^{M N} \partial_{N} \phi-3 \mathcal{M}^{M N} \partial_{M} \phi \partial_{N} \phi=0$,

which is exactly the dilaton equation $\operatorname{tr}(\mathcal{T})=0$ as following from the OPE.

\section{Prospects}

This paper provides a different approach to $\alpha^{\prime}$ corrections of low-energy string actions. Traditionally these corrections have been gleaned from the string theory S-matrix, and then terms are constructed for the low-energy theory that reproduce such S-matrix results. In this paper $\alpha^{\prime}$ corrections are seen as required by a modified gauge structure. They are predicted, or at least constrained by a symmetry principle. We want to emphasize that our use of the double field theory approach does not mean that the results are only valid for compactified theories. The $\alpha^{\prime}$ information obtained is background independent. 
$\alpha^{\prime}$-geometry. In string theory $\alpha^{\prime}$ corrections are part of the (target space) classical theory. More precisely, classical closed string field theory includes $\alpha^{\prime}$ corrections of all orders. For the massless sector, the $\alpha^{\prime}$ corrections parameterize the way in which the string theory differs from the two-derivative Einstein action coupled to a two-form and a dilaton. It therefore has been reasonable to expect that the appropriate geometry of string theory should be an $\alpha^{\prime}$-deformation of Riemannian geometry. The incorporation of T-duality has forced on us a doubled geometry that can be viewed as a mild extension of generalized geometry. This is the case even for the two-derivative theory. This geometry has an inner product, a C-bracket and generalized Lie derivatives, that upon reduction from $\mathrm{D}+\mathrm{D}$ to $\mathrm{D}$ dimensions give the inner product, the Courant bracket and the Lie derivatives of generalized geometry. The $\alpha^{\prime}$ corrections are nontrivial deformations of the geometry. The inner product and C-bracket acquire a correction that is in fact linear in $\alpha^{\prime}$. Gauge transformations or generalized Lie derivatives acquire a linear correction for a vector field and a linear plus quadratic correction for a two-tensor. This is a "complete" deformation: the bracket is fully consistent (has a trivial Jacobiator) without higher-order corrections, and the commutator of generalized Lie derivatives gives precisely the Lie derivative along the C-bracket of the input gauge parameters. We want to emphasize that the C-bracket does not allow higher-order $\alpha^{\prime}$ corrections consistent with linearity in its arguments, so the correction we have is unique. This indicates that the above represents a first step in the construction of the $\alpha^{\prime}$-geometry. Intriguingly, the corrections to all these structures do not vanish when reduced from the doubled manifold to D dimensions. Therefore, they define an apparently unknown deformation of the Courant bracket and other structures in generalized geometry.

Double metric $\mathcal{M}$. The generalized metric $\mathcal{H}$ of the doubled manifold was a dualitycovariant gravitational field variable for the two-derivative theory. Surprisingly, the relevant OPE's indicated that the constraint $\mathcal{H}^{2}=1$ satisfied by this metric cannot be preserved when considering $\alpha^{\prime}$ corrections. We were thus led to consider a double metric $\mathcal{M}$, an unconstrained extension of the generalized metric. Just like ordinary metrics, $\mathcal{M}$ does not satisfy an algebraic constraint. But even more is true: we do not need to assume $\mathcal{M}$ is invertible to define the action, yet it is invertible on-shell as a consequence of its field equation $\mathcal{M}^{2}=1+\ldots$. The straightforward emergence of $\mathcal{M}$ and the simplicity of the action suggests that $\mathcal{M}$ is a natural variable for the fundamental description of gravitational degrees of freedom in string theory.

A new consistent truncation of string theory? We have constructed an $\alpha^{\prime}$ deformation of the low-energy effective action. In terms of the gravitational variable $\mathcal{M}$ and the dilaton, the action and field equations contain bounded powers of $\alpha^{\prime}$. In terms of $(g, b, \phi)$, the equations of motion and the action presumably contain terms to all orders in $\alpha^{\prime}$. The obvious question is: is this the exact effective action of string theory for the massless sector? It seems not: the four-point and higher point amplitudes in this theory are not expected to contain the poles associated with the massive string states. The theory is, however, fully consistent: all $\alpha^{\prime}$ dependent gauge symmetries are exact invariances. This indicates that this theory is a consistent truncation of string theory in which some 
of the stringy non-locality has been eliminated. The higher-derivative contributions that remain can be perhaps traced to those in the three-closed-string vertex. With a suitable off-shell definition of the vertex (not the one used in closed string field theory, in fact) the massless field three-vertices contain only finite number of derivatives (two, four, and six for the case of three gravitons). The theory we have may be the consistent completion of such cubic theory.

A new worldsheet theory? We extended double field theory consistently to higher order(s) in $\alpha^{\prime}$. The method is a "complete" result for a system related to the usual string theory, but employing free, chiral bosons. Further investigation is required to determine how this chiral string relates to the usual string beyond the classical level. It would be useful to have a derivation of this theory obtained by gauge fixing of the standard first-quantized action. Such gauge-fixing would teach us how to introduce ghost fields, which are needed beyond the classical level discussed in this paper. In this theory the strong constraint ensures that the OPE of fields is nonsingular. Thus the derivation of the field equations from conformal invariance is greatly simplified, as compared to the usual calculation of beta functions [4]. It also suggests a new string field theory based on the BRST operator for this chiral Virasoro algebra.

Covariant derivatives, torsions, and curvatures. For further clarification of the geometry, the inclusion of the Lorentz current algebra will allow for true covariant derivatives in a vielbein formalism (also required for supersymmetry) [36]: $\alpha^{\prime}$ corrections to torsions, curvatures, and local Lorentz transformations will then automatically follow by the same methods used in this paper. The corresponding expressions should exist in terms of the generalized metric used here and generalized Christoffel symbols as an extension of the methods in [23] (eqs. (1.5) and (1.6)). Some components of Riemann would still be undetermined, since suitable generalized constraints are still going to fail to fix the connection completely. But just as in the case of the two-derivative theory, the contractions that give the scalar curvature may eliminate all undetermined components. If this is so, the action density would simply be the "scalar curvature" associated with the $\alpha^{\prime}$-corrected Riemann.

Relation to conventional field theory. It is of great interest to see how the theory given here is related to one that has a metric $g$ and a two-form field $b$ with conventional gauge transformations - of course, by sacrificing manifest T-duality. This assumes that the $\alpha^{\prime}$-deformation of our gauge structures can be trivialized using T-duality violating and gauge non-covariant field redefinitions. This seems very plausible, but should be investigated. Naively, one may try to identify the conventional fields $g$ and $b$ via the generalized metric $\mathcal{H}$, as the latter is naturally parametrized in terms of these fields. As noted at the end of section 7.2 , however, writing $\mathcal{M}$ in terms of $\mathcal{H}$ was only possible on-shell. Therefore, from this starting point one cannot derive off-shell gauge transformations of $g$ and $b$, nor an off-shell action for these fields. Perhaps it will be possible to identify $g$ and $b$ directly as components of the double metric $\mathcal{M}$, which would also contain a number of auxiliary field degrees of freedom, but this remains an open question. Some progress may be possible in a perturbative analysis around a constant background, giving a relation between dou- 
ble metric fluctuations and the (background-dependent) field variables in closed string field theory. Partial results along these lines have been obtained, and we hope to report on them in the near future. A related question is the appearance of higher-order curvature terms in the action. To this end we note that the cubic term of the action is essentially the same as the most singular $\left(1 / z^{6}\right)$ term in the OPE of three operators $\mathcal{T}$ 's at three different $z$ 's. But this is the same calculation that gives the three-point function of the corresponding vertex operators (the operators minus their vacuum pieces, with the ghosts cancelling the $1 / z^{6}$ ). The result of the latter is the cubic pieces of $R+R^{2}+R^{3}$ (where " $R$ " stands for the Riemann tensor). Our action is expected to yield the T-duality covariantization of this.

Relation to conventional string theory. We have dealt with genuine string theory, which is evident from our starting point, where the equations of motion came from the closure of the Virasoro algebra and the action was written to give such equations of motion. The formalism, however, allows one to define other gauge invariant terms that could be added to the action, at the price of changing the field equations and perhaps losing the connection to string theory. Such alternative actions may be of some interest. On the other hand, the existence of these higher-derivative gauge-invariant terms could allow the construction of those $\alpha^{\prime}$ contributions that turn the present theory into one that reproduces the dual amplitudes of string theory.

Other. The action and field equations found here are unusual in that they contain both dynamics and algebraic constraints from the same field (no Lagrange multipliers or auxiliary fields). This is similar to the decomposition of gauge fields into gauge, auxiliary, and dynamical components in a lightcone gauge, but here the decomposition is local and Lorentz covariant. It would be interesting to see if this new concept can be extended to other systems. An obvious avenue of extension of the current results is to superstrings, whose classical treatment was begun in [10-12]. It may also be interesting to consider the inclusion of higher weight operators describing higher spin fields.

\section{Acknowledgments}

$\mathrm{OH}$ and BZ would like to thank Ashoke Sen for early discussions on the problem of $\alpha^{\prime}$ corrections and T-duality. WS was supported in part by National Science Foundation Grant No. PHY-0969739. OH was supported by the DFG Transregional Collaborative Research Centre TRR 33 and the DFG cluster of excellence "Origin and Structure of the Universe".

\section{A Quadratic identities}

$$
\begin{aligned}
\mathcal{O}_{2} \circ_{w} \mathcal{O}_{1} & =(-1)^{w_{1}+w_{2}-w} e^{-\mathcal{L}_{\mathcal{O}_{1}} \circ_{w}} \mathcal{O}_{2} \\
\mathcal{O}_{1}^{\prime} \circ_{w} \mathcal{O}_{2} & =\left(w-w_{1}-w_{2}\right) \mathcal{O}_{1} \circ_{w} \mathcal{O}_{2}, \\
\left(\mathcal{O}_{1} \circ_{w} \mathcal{O}_{2}\right)^{\prime} & =\mathcal{O}_{1}^{\prime} \circ_{w+1} \mathcal{O}_{2}+\mathcal{O}_{1} \circ_{w+1} \mathcal{O}_{2}^{\prime}
\end{aligned}
$$




$$
\begin{aligned}
\mathcal{S} \circ_{w_{\mathcal{O}}+1} \mathcal{O} & =\mathcal{O}^{\prime} \\
\mathcal{S} \circ_{w_{\mathcal{O}}} \mathcal{O} & =w_{\mathcal{O}} \mathcal{O} \\
\mathcal{S} \circ_{w_{\mathcal{O}}-1} \mathcal{O} & \equiv \operatorname{div}(\mathcal{O}) \\
\mathcal{S} \circ_{w_{\mathcal{O}}-2} \mathcal{O} & \equiv \frac{1}{2} \operatorname{tr}(\mathcal{O})
\end{aligned}
$$

\section{Examples:}

$$
\begin{aligned}
\operatorname{tr}\left(\mathcal{O}^{\prime}\right) & =(\operatorname{tr} \mathcal{O})^{\prime}+6 \operatorname{div} \mathcal{O}, \\
\operatorname{div}\left(\mathcal{O}^{\prime}\right) & =(\operatorname{div} \mathcal{O})^{\prime}+2 w_{\mathcal{O}} \mathcal{O} \\
\operatorname{tr} V=\operatorname{tr} f & =\operatorname{div} f=0
\end{aligned}
$$

$\operatorname{tr}\left(V^{\prime}\right)=6 \operatorname{div} V, \quad \operatorname{div}\left(V^{\prime}\right)=2 V+(\operatorname{div} V)^{\prime}, \quad \operatorname{div}\left(f^{\prime}\right)=0$,

$\operatorname{div}\left(f^{\prime \prime}\right)=2 f^{\prime}, \quad \operatorname{tr}\left(f^{\prime \prime}\right)=0$

\section{B Cubic identities}

$$
\begin{aligned}
\mathcal{O}_{1} \circ_{\hat{w}}\left(\mathcal{O}_{2} \circ_{w_{2}+w_{3}-w} \mathcal{O}_{3}\right)-\mathcal{O}_{2} \circ_{\hat{w}}\left(\mathcal{O}_{1} \circ_{w_{1}+w_{3}-w^{\prime}} \mathcal{O}_{3}\right) \\
=\sum_{w^{\prime \prime}=1}^{w^{\prime}}\left(\begin{array}{l}
w^{\prime}-1 \\
w^{\prime \prime}-1
\end{array}\right)\left(\mathcal{O}_{1} \circ_{w_{1}+w_{2}-w^{\prime \prime}} \mathcal{O}_{2}\right) \circ_{\hat{w}} \mathcal{O}_{3} \\
\hat{w}+w+w^{\prime}=w_{1}+w_{2}+w_{3}, \quad w^{\prime} \geq 1
\end{aligned}
$$

\section{Examples:}

- $w_{1}=w_{2}=w_{3}=2$ :

- $w=2, w^{\prime}=4, \hat{w}=0$

$$
\begin{aligned}
T_{1} \circ_{0}\left(T_{2} \circ_{2} T_{3}\right)=T_{2} \circ_{0}\left(T_{1} \circ_{0} T_{3}\right)+\left(T_{1} \circ_{0} T_{2}\right) \circ_{0} T_{3} \\
+3\left(T_{1} \circ_{1} T_{2}\right) \circ_{0} T_{3}+3\left(T_{1} \circ_{2} T_{2}\right) \circ_{0} T_{3}+\left(T_{1} \circ_{3} T_{2}\right) \circ_{0} T_{3}
\end{aligned}
$$

- $w=2, w^{\prime}=3, \hat{w}=1$

$$
T_{1} \circ_{1}\left(T_{2} \circ_{2} T_{3}\right)=T_{2} \circ_{1}\left(T_{1} \circ_{1} T_{3}\right)+\left(T_{1} \circ_{3} T_{2}\right) \circ_{1} T_{3}+2\left(T_{1} \circ_{2} T_{2}\right) \circ_{1} T_{3}+\left(T_{1} \circ_{1} T_{2}\right) \circ_{1} T_{3}
$$

The two equations above with the first tensor taken to be $\mathcal{S}$ give

$$
\begin{aligned}
& \frac{1}{2} \operatorname{tr}\left(T_{1} \circ_{2} T_{2}\right)=\frac{1}{2} T_{1} \circ_{0}(\left.\operatorname{tr} T_{2}\right)+\frac{1}{2}\left(\operatorname{tr} T_{1}\right) \circ_{0} T_{2} \\
&+3\left(\operatorname{div} T_{1}\right) \circ_{0} T_{2}+6 T_{1} \circ_{0} T_{2}+T_{1}^{\prime} \circ_{0} T_{2} \\
& \operatorname{div}\left(T_{1} \circ_{2} T_{2}\right)=T_{1} \circ_{1}\left(\operatorname{div} T_{2}\right)+T_{1}^{\prime} \circ_{1} T_{2}+4 T_{1} \circ_{1} T_{2}+\left(\operatorname{div} T_{1}\right) \circ_{1} T_{2}
\end{aligned}
$$


- $w=5, w^{\prime}=1$

$$
0=T_{2} \circ_{0}\left(T_{1} \circ_{3} T_{3}\right)+\left(T_{1} \circ_{3} T_{2}\right) \circ_{0} T_{3}
$$

- $w=4, w^{\prime}=1$

$$
T_{1} \circ_{1}\left(T_{2} \circ_{0} T_{3}\right)=T_{2} \circ_{1}\left(T_{1} \circ_{3} T_{3}\right)+\left(T_{1} \circ_{3} T_{2}\right) \circ_{1} T_{3}
$$

- $w=4, w^{\prime}=2$

$$
T_{1} \circ_{0}\left(T_{2} \circ_{0} T_{3}\right)=T_{2} \circ_{0}\left(T_{1} \circ_{2} T_{3}\right)+\left(T_{1} \circ_{2} T_{2}\right) \circ_{0} T_{3}+\left(T_{1} \circ_{3} T_{2}\right) \circ_{0} T_{3}
$$

- $w=2, w^{\prime}=4$

$$
\begin{aligned}
T_{1} \circ_{0}\left(T_{2} \circ_{2} T_{3}\right)= & T_{2} \circ_{0}\left(T_{1} \circ_{0} T_{3}\right)+\left(T_{1} \circ_{0} T_{2}\right) \circ_{0} T_{3} \\
& +3\left(T_{1} \circ_{1} T_{2}\right) \circ_{0} T_{3}+3\left(T_{1} \circ_{2} T_{2}\right) \circ_{0} T_{3}+\left(T_{1} \circ_{3} T_{2}\right) \circ_{0} T_{3}
\end{aligned}
$$

- $w=3, w^{\prime}=3$

$$
\begin{gathered}
T_{1} \circ_{0}\left(T_{2} \circ_{1} T_{3}\right)=T_{2} \circ_{0}\left(T_{1} \circ_{1} T_{3}\right)+\left(T_{1} \circ_{1} T_{2}\right) \circ_{0} T_{3} \\
+2\left(T_{1} \circ_{2} T_{2}\right) \circ_{0} T_{3}+\left(T_{1} \circ_{3} T_{2}\right) \circ_{0} T_{3} \\
\operatorname{div}\left(T_{2} \circ_{1} T_{3}\right)=\left\langle T_{2} \mid \operatorname{div}\left(T_{3}\right)\right\rangle+\left\langle\operatorname{div}\left(T_{2}\right) \mid T_{3}\right\rangle
\end{gathered}
$$

\section{Evaluated products}

$$
\begin{aligned}
\operatorname{tr}(T) & =\eta^{M N} T_{M N}-3\left(T^{M N} \partial_{M} \partial_{N} \phi+\partial \cdot \hat{T}+\hat{T} \cdot \partial \phi\right) \\
\operatorname{div}(T)^{M} & =\partial_{N} T^{M N}+T^{M N} \partial_{N} \phi-\frac{1}{2} T^{N P} \partial_{N} \partial_{P} \partial^{M} \phi-\hat{T}^{M}-\frac{1}{2} \partial^{M}(\partial \cdot \hat{T}+\hat{T} \cdot \partial \phi) \\
\operatorname{div}(V)= & \partial \cdot V+V \cdot \partial \phi \\
\langle T \mid f\rangle= & \frac{1}{2}\left(T^{M N} \partial_{M} \partial_{N} f+\hat{T}^{M} \partial_{M} f\right) \\
\left(T \circ_{1} f\right)^{M}= & T^{M N} \partial_{N} f+\frac{1}{2}\left(\partial^{M} T^{N P}\right) \partial_{N} \partial_{P} f \\
\left(f \circ_{1} T\right)^{M}= & -T^{M N} \partial_{N} f+\frac{1}{2} T^{N P} \partial_{N} \partial_{P} \partial^{M} f+\frac{1}{2} \partial^{M}\left(\hat{T}^{N} \partial_{N} f\right) \\
\langle V \mid T\rangle= & -\langle V \mid \hat{T}\rangle-T^{M N} \partial_{M} V_{N}+\frac{1}{2}\left(\partial^{M} T^{N P}\right) \partial_{N} \partial_{P} V_{M} \\
\left(V \circ_{1} T\right)^{M}= & T^{M N} V_{N}-\frac{1}{2}\left([V, \widehat{T}]_{D}^{M}+\partial^{M}\langle V \mid \hat{T}\rangle\right)+\frac{1}{2} T^{N P} \partial_{N} \partial_{P} V^{M}-T^{N P} \partial^{M} \partial_{N} V_{P} \\
& -\left(\partial^{N} T^{M P}\right) \partial_{P} V_{N}+\frac{1}{2}\left(\partial^{N} T^{P Q}\right) \partial^{M} \partial_{P} \partial_{Q} V_{N}
\end{aligned}
$$


$\left(V \circ_{2} T\right.$ is in (4.60). Here $\langle V \mid \hat{T}\rangle$ means the inner product from treating $\hat{T}^{M} Z_{M}$ as if it were a vector and not a pseudovector.)

$$
\begin{aligned}
T_{1} \bullet T_{2} T_{2}= & -\frac{1}{4} T_{1}{ }^{P Q} \stackrel{\leftrightarrow}{\partial}_{M} T_{2 P Q}+\left(T_{1}{ }^{L K} \partial_{L} T_{2 K M}-T_{2}{ }^{L K} \partial_{L} T_{1 K M}\right)+\frac{1}{2} \partial_{P} T_{1}{ }^{L K} \stackrel{\leftrightarrow}{\partial}_{M} \partial_{L} T_{2 K}{ }^{P} \\
& +\frac{1}{2}\left(\partial_{K} T_{2}{ }^{P Q} \partial_{P} \partial_{Q} T_{1}{ }^{K}{ }_{M}-\partial_{K} T_{1}{ }^{P Q} \partial_{P} \partial_{Q} T_{2}{ }^{K}{ }_{M}\right)-\frac{1}{8} \partial_{P} \partial_{Q} T_{1}{ }^{K L} \stackrel{\leftrightarrow}{\partial}_{M} \partial_{K} \partial_{L} T_{2}{ }^{P Q} \\
& -\frac{1}{2}\left(\left[\hat{T}_{1}, \hat{T}_{2}\right]_{C}\right)_{M}+\hat{T}_{1}{ }^{K} T_{2 K M}-\partial_{P} \hat{T}_{1}{ }^{K} \partial_{K} T_{2}{ }^{P}{ }_{M}-\partial_{M} \partial_{P} \hat{T}_{1}{ }^{K} T_{2 K}{ }^{P}-\hat{T}_{2}{ }^{K} T_{1 K M} \\
& +\partial_{P} \hat{T}_{2}{ }^{K} \partial_{K} T_{1}{ }^{P}{ }_{M}+\partial_{M} \partial_{P} \hat{T}_{2}{ }^{K} T_{1 K}{ }^{P}+\frac{1}{2} \partial_{P} \partial_{Q} \hat{T}_{1 M} T_{2}{ }^{P Q} \\
& +\frac{1}{2} \partial_{M} \partial_{P} \partial_{Q} \hat{T}_{1}{ }^{K} \partial_{K} T_{2}{ }^{P Q}-\frac{1}{2} \partial_{P} \partial_{Q} \hat{T}_{2 M} T_{1}{ }^{P Q}-\frac{1}{2} \partial_{M} \partial_{P} \partial_{Q} \hat{T}_{2}{ }^{K} \partial_{K} T_{1}{ }^{P Q} \\
& +\partial_{M}\left[\frac{3}{4}\left(\partial_{P} \hat{T}_{1}{ }^{K} T_{2}{ }^{P}-\partial_{P} \hat{T}_{2}{ }^{K} T_{1 K}{ }^{P}\right)\right. \\
& \left.\quad-\frac{3}{8}\left(\partial_{P} \partial_{Q} \hat{T}_{1}{ }^{K} \partial_{K} T_{2}{ }^{P Q}-\partial_{P} \partial_{Q} \hat{T}_{2}{ }^{K} \partial_{K} T_{1}{ }^{P Q}\right)\right]
\end{aligned}
$$

\section{Alternate projection}

We consider here a different, tilde projection from operators $\mathcal{O}$ to operators $\tilde{\mathcal{O}}$. There is also a different divergence operator $\widetilde{\mathrm{Div}}$ associated with this projection. Although we do not have a specific application in mind, this projection is in some ways simpler than the overline projection.

The operator $\tilde{\mathcal{O}}$ is defined implicitly by the following relation

$$
(\tilde{\mathcal{O}})^{\prime} \equiv \frac{1}{w_{\mathcal{O}}-1} \mathcal{O} \circ_{w_{\mathcal{O}}+1} \mathcal{S}
$$

The derivative identity shows that the above implies that

$$
\widetilde{\left(\mathcal{O}^{\prime}\right)}=0
$$

We can evaluate $\tilde{\mathcal{O}}$ in (D.1) by use of the symmetry identity which confirms that the right-hand side is a $z$-derivative. We then get

$$
\tilde{\mathcal{O}}=\mathcal{O}+\frac{1}{w_{\mathcal{O}}-1} \sum_{w^{\prime}=1}^{w_{\mathcal{O}}} \frac{(-1)^{w^{\prime}}}{\left(w^{\prime}+1\right) !}\left(\mathcal{S} \circ_{w_{\mathcal{O}}-w^{\prime}} \mathcal{O}\right)^{\left(w^{\prime}\right)}, \quad \tilde{\tilde{\mathcal{O}}}=\tilde{\mathcal{O}}
$$

For the new divergence we define

$$
\begin{aligned}
\widetilde{\operatorname{Div}}(\mathcal{O}) & \equiv 2 \sum_{w^{\prime}=0}^{w_{\mathcal{O}}-1} \frac{(-1)^{w^{\prime}}}{\left(w^{\prime}+2\right) !}\left(\mathcal{S} \circ_{w_{\mathcal{O}}-w^{\prime}-1} \mathcal{O}\right)^{\left(w^{\prime}\right)}=\operatorname{div}(\mathcal{O})-\frac{1}{6}[\operatorname{tr}(\mathcal{O})]^{\prime}+\ldots \\
\tilde{\mathcal{O}} & =\mathcal{O}-\frac{1}{2\left(w_{\mathcal{O}}-1\right)}(\widetilde{\operatorname{Div}} \mathcal{O})^{\prime}, \quad \widetilde{\operatorname{Div}}(\tilde{\mathcal{O}})=0, \quad \widetilde{\operatorname{Div}}\left(\mathcal{O}^{\prime}\right)=2 w_{\mathcal{O}} \mathcal{O}
\end{aligned}
$$


In particular,

$$
\begin{aligned}
\operatorname{tr} \tilde{T} & =\operatorname{tr} T-3 \operatorname{div}^{2} T=\operatorname{tr} \bar{T} \\
& =\eta^{M N} T_{M N}-3 \partial_{M}\left(\partial_{N} T^{M N}+2 T^{M N} \partial_{N} \phi\right)-3 T^{M N} \partial_{M} \phi \partial_{N} \phi \\
(\widetilde{\operatorname{Div} T})^{M} & =\left[\operatorname{div} T-\frac{1}{6}(\operatorname{tr} T)^{\prime}\right]^{M} \\
& =-\hat{T}^{M}+\partial_{N} T^{M N}-\frac{1}{6} \partial^{M} \eta^{N P} T_{N P}+T^{M N} \partial_{N} \phi+\frac{1}{2}\left(\partial^{M} T^{N P}\right) \partial_{N} \partial_{P} \phi \\
\widetilde{T} & =T-\frac{1}{2}(\widetilde{\operatorname{Div} T})^{\prime}=\bar{T}+\frac{1}{12}(\operatorname{tr} \bar{T})^{\prime \prime} \\
& =\frac{1}{2} T_{M N} Z^{M} Z^{N}-\frac{1}{2}\left\{\left[\partial_{N} T^{M N}-\frac{1}{6} \partial^{M} \eta^{N P} T_{N P}+T^{M N} \partial_{N} \phi\right.\right. \\
& \left.\left.+\frac{1}{2}\left(\partial^{M} T^{N P}\right) \partial_{N} \partial_{P} \phi\right] Z_{M}\right\}^{\prime}
\end{aligned}
$$

We also have the trivial cases

$$
\tilde{\mathcal{S}}=\mathcal{S} \quad \Rightarrow \quad \operatorname{tr} \tilde{\mathcal{S}}=2 D, \quad \widetilde{\operatorname{Div}} \mathcal{S}=0
$$

For the variation of an arbitrary projected operator, explicit evaluation yields

$$
\delta \tilde{\mathcal{O}}=\widetilde{\delta \mathcal{O}}-\frac{1}{4\left(w_{\mathcal{O}}-1\right)}\left(\mathcal{O} \circ_{w_{\mathcal{O}}-1} \delta \phi\right)^{\prime}
$$

Open Access. This article is distributed under the terms of the Creative Commons Attribution License (CC-BY 4.0), which permits any use, distribution and reproduction in any medium, provided the original author(s) and source are credited.

\section{References}

[1] W. Siegel, Manifest Lorentz invariance sometimes requires nonlinearity, Nucl. Phys. B 238 (1984) 307 [inSPIRE].

[2] E. Fradkin and A.A. Tseytlin, Effective field theory from quantized strings, Phys. Lett. B 158 (1985) 316 [inSPIRE].

[3] E. Fradkin and A.A. Tseytlin, Quantum string theory effective action, Nucl. Phys. B 261 (1985) 1 [INSPIRE].

[4] T. Banks, D. Nemeschansky and A. Sen, Dilaton coupling and BRST quantization of bosonic strings, Nucl. Phys. B 277 (1986) 67 [INSPIRE].

[5] R. Floreanini and R. Jackiw, Selfdual fields as charge density solitons, Phys. Rev. Lett. 59 (1987) 1873 [InSPIRE].

[6] C.M. Hull, Covariant quantization of chiral bosons and anomaly cancellation, Phys. Lett. B 206 (1988) 234 [inSPIRE].

[7] M. Duff, Duality rotations in string theory, Nucl. Phys. B 335 (1990) 610 [INSPIRE].

[8] A.A. Tseytlin, Duality symmetric formulation of string world sheet dynamics, Phys. Lett. B 242 (1990) 163 [INSPIRE]. 
[9] A.A. Tseytlin, Duality symmetric closed string theory and interacting chiral scalars, Nucl. Phys. B 350 (1991) 395 [inSPIRE].

[10] W. Siegel, Two vierbein formalism for string inspired axionic gravity, Phys. Rev. D 47 (1993) 5453 [hep-th/9302036] [INSPIRE].

[11] W. Siegel, Superspace duality in low-energy superstrings, Phys. Rev. D 48 (1993) 2826 [hep-th/9305073] [INSPIRE].

[12] W. Siegel, Manifest duality in low-energy superstrings, hep-th/9308133 [INSPIRE].

[13] W. Siegel, Untwisting the twistor superstring, hep-th/0404255 [INSPIRE].

[14] C. Hull and B. Zwiebach, Double field theory, JHEP 09 (2009) 099 [arXiv:0904.4664] [INSPIRE].

[15] B. Zwiebach, Closed string field theory: quantum action and the B- $V$ master equation, Nucl. Phys. B 390 (1993) 33 [hep-th/9206084] [INSPIRE].

[16] T. Kugo and B. Zwiebach, Target space duality as a symmetry of string field theory, Prog. Theor. Phys. 87 (1992) 801 [hep-th/9201040] [INSPIRE].

[17] C. Hull and B. Zwiebach, The gauge algebra of double field theory and Courant brackets, JHEP 09 (2009) 090 [arXiv: 0908.1792] [INSPIRE].

[18] O. Hohm, C. Hull and B. Zwiebach, Generalized metric formulation of double field theory, JHEP 08 (2010) 008 [arXiv: 1006.4823] [INSPIRE].

[19] O. Hohm, C. Hull and B. Zwiebach, Background independent action for double field theory, JHEP 07 (2010) 016 [arXiv: 1003.5027] [INSPIRE].

[20] O. Hohm and S.K. Kwak, Frame-like geometry of double field theory, J. Phys. A 44 (2011) 085404 [arXiv:1011.4101] [InSPIRE].

[21] O. Hohm and B. Zwiebach, On the Riemann tensor in double field theory, JHEP 05 (2012) 126 [arXiv:1112.5296] [INSPIRE].

[22] O. Hohm and B. Zwiebach, Large gauge transformations in double field theory, JHEP 02 (2013) 075 [arXiv:1207.4198] [INSPIRE].

[23] O. Hohm and B. Zwiebach, Towards an invariant geometry of double field theory, J. Math. Phys. 54 (2013) 032303 [arXiv:1212.1736] [INSPIRE].

[24] I. Jeon, K. Lee and J.-H. Park, Differential geometry with a projection: Application to double field theory, JHEP 04 (2011) 014 [arXiv: 1011.1324] [INSPIRE].

[25] I. Jeon, K. Lee and J.-H. Park, Stringy differential geometry, beyond Riemann, Phys. Rev. D 84 (2011) 044022 [arXiv: 1105.6294] [INSPIRE].

[26] N. Hitchin, Generalized Calabi-Yau manifolds, Quart. J. Math. Oxford Ser. 54 (2003) 281 [math/0209099] [INSPIRE].

[27] M. Gualtieri, Generalized complex geometry, math/0401221 [INSPIRE].

[28] M. Gualtieri, Branes on Poisson varieties, arXiv:0710.2719 [INSPIRE].

[29] O. Hohm and S.K. Kwak, Massive type II in double field theory, JHEP 11 (2011) 086 [arXiv: 1108.4937] [INSPIRE].

[30] O. Hohm and S.K. Kwak, Double field theory formulation of heterotic strings, JHEP 06 (2011) 096 [arXiv:1103.2136] [INSPIRE]. 
[31] O. Hohm, S.K. Kwak and B. Zwiebach, Double field theory of type II strings, JHEP 09 (2011) 013 [arXiv: 1107.0008] [INSPIRE].

[32] O. Hohm, S.K. Kwak and B. Zwiebach, Unification of type II strings and T-duality, Phys. Rev. Lett. 107 (2011) 171603 [arXiv:1106.5452] [INSPIRE].

[33] A. Coimbra, C. Strickland-Constable and D. Waldram, Supergravity as generalised geometry I: type II theories, JHEP 11 (2011) 091 [arXiv: 1107.1733] [INSPIRE].

[34] O. Hohm and S.K. Kwak, $N=1$ supersymmetric double field theory, JHEP 03 (2012) 080 [arXiv: 1111.7293] [INSPIRE].

[35] I. Jeon, K. Lee and J.-H. Park, Supersymmetric double field theory: stringy reformulation of supergravity, Phys. Rev. D 85 (2012) 081501 [Erratum ibid. D 86 (2012) 089903] [arXiv: 1112.0069] [INSPIRE].

[36] W. Siegel, New superspaces/algebras for superparticles/strings, arXiv:1106.1585 [INSPIRE].

[37] G. Aldazabal, W. Baron, D. Marques and C. Núñez, The effective action of double field theory, JHEP 11 (2011) 052 [Erratum ibid. 1111 (2011) 109] [arXiv:1109.0290] [INSPIRE].

[38] D. Geissbuhler, Double field theory and $N=4$ gauged supergravity, JHEP 11 (2011) 116 [arXiv: 1109.4280] [INSPIRE].

[39] M. Graña and D. Marques, Gauged double field theory, JHEP 04 (2012) 020 [arXiv: 1201.2924] [INSPIRE].

[40] D. Geissbuhler, D. Marques, C. Núñez and V. Penas, Exploring double field theory, JHEP 06 (2013) 101 [arXiv:1304.1472] [INSPIRE].

[41] D. Andriot, O. Hohm, M. Larfors, D. Lüst and P. Patalong, A geometric action for non-geometric fluxes, Phys. Rev. Lett. 108 (2012) 261602 [arXiv:1202.3060] [INSPIRE].

[42] D. Andriot, O. Hohm, M. Larfors, D. Lüst and P. Patalong, Non-geometric fluxes in supergravity and double field theory, Fortsch. Phys. 60 (2012) 1150 [arXiv:1204.1979] [INSPIRE].

[43] C. Hillmann, Generalized $E_{7(7)}$ coset dynamics and $D=11$ supergravity, JHEP 03 (2009) 135 [arXiv:0901.1581] [INSPIRE].

[44] D.S. Berman and M.J. Perry, Generalized geometry and M-theory, JHEP 06 (2011) 074 [arXiv: 1008.1763] [INSPIRE].

[45] A. Coimbra, C. Strickland-Constable and D. Waldram, $E_{d(d)} \times \mathbb{R}^{+}$generalised geometry, connections and $M$-theory, arXiv:1112.3989 [INSPIRE].

[46] G. Aldazabal, D. Marques and C. Núñez, Double field theory: a pedagogical review, Class. Quant. Grav. 30 (2013) 163001 [arXiv:1305.1907] [INSPIRE].

[47] A. Sen, $O(d) \times O(d)$ symmetry of the space of cosmological solutions in string theory, scale factor duality and two-dimensional black holes, Phys. Lett. B 271 (1991) 295 [INSPIRE].

[48] K.A. Meissner, Symmetries of higher order string gravity actions, Phys. Lett. B 392 (1997) 298 [hep-th/9610131] [INSPIRE].

[49] N. Kaloper and K.A. Meissner, Duality beyond the first loop, Phys. Rev. D 56 (1997) 7940 [hep-th/9705193] [INSPIRE]. 